\title{
Design, Synthesis, Insecticidal Activity of Novel Triazone Derivatives Containing Sulfonamide or Sulfonimide Moieties
}

\author{
Yan Yang ${ }^{1,2}$, Yuxiu Liu ${ }^{1}$, Hongjian Song ${ }^{1 *}$, Yongqiang Li $^{1}$, Qingmin Wang ${ }^{1 *}$ \\ ${ }^{1}$ State Key Laboratory of Elemento-Organic Chemistry, College of Chemistry, \\ Frontiers Science Center for New Organic Matter, Nankai University, Tianjin 300071, \\ People's Republic of China. \\ ${ }^{2}$ College of Biomedical Engineering, Taiyuan University of Technology, Taiyuan \\ 030024, People's Republic of China.
}




\section{Table of Contents}

1. Experimental operating steps and physical data....................S3-S16

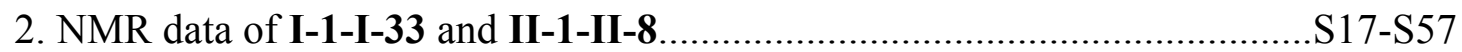

3. Detailed procedures for the foliar contact activity against bean aphid (Aphis

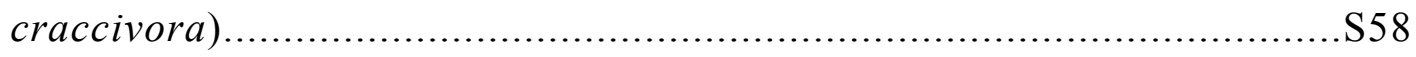

4. Detailed procedures for the larvacidal activities against mosquito larvae (Culex

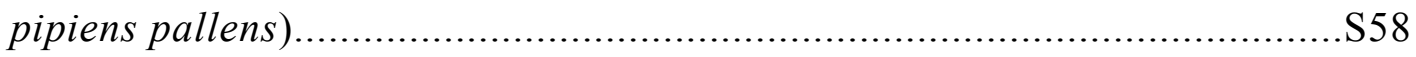

5. Detailed procedures for the stomach toxicity against cotton bollworm (Helicoverpa armigera), corn borer (Ostrinia nubilalis) and oriental armyworm (Mythimna

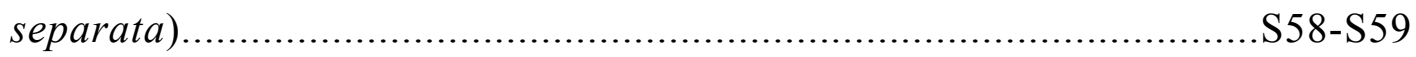

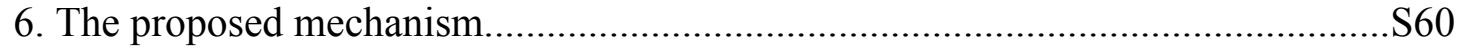




\section{Experimental Operating Steps and Physical Data}

Synthesis of $\quad N$-(6-methyl-3-oxo-2,3-dihydro-1,2,4-triazin-4(5H)yl)methanesulfonamide (I-1).

Amino-6-methyl-4,5-dihydro-1,2,4-triazin-3(2H)-one $(\mathbf{1} ; 0.26 \mathrm{~g}, 2 \mathrm{mmol})$, pyridine ( $0.4 \mathrm{~mL}, 2.5$ equiv), and DMAP $(2.44 \mathrm{mg})$ were dissolved in $2.0 \mathrm{~mL}$ of DCM, and the resulting white suspension was stirred at room temperature for $15 \mathrm{~min}$. Then methanesulfonyl chloride (2-1; $0.24 \mathrm{~g}, 1.05$ equiv) dissolved in $1.7 \mathrm{~mL}$ of DCM was added dropwise within about $5 \mathrm{~min}$. About $5 \mathrm{~min}$ after the start of the addition of 2 , the reaction mixture gradually became a yellow solution. After the addition was completed, the reaction continues stirring for another $10 \mathrm{~h}$ at room temperature, at which point TLC (60:1 DCM/methanol) indicated that the reaction was complete. Note that a white suspension formed during the course of the reaction. DCM $(3.0 \mathrm{~mL})$ and dilute $\mathrm{HCl}$ (4.4 mL, $1 \mathrm{~mol} / \mathrm{L})$ were added, and the resulting two phases were separated in a separatory funnel. The aqueous phase was extracted with DCM $(2.0 \mathrm{~mL} \times 3)$, and the combined organic phases were washed with water $(5.0 \mathrm{~mL})$ and saturated brine $(5.0$ $\mathrm{mL}$ ), dried with anhydrous sodium sulfate, and filtered. Evaporation of the filtrate in vacuo gave I-1 (0.4 g, 98\% yield) as a yellow solid. Mp 191-192 ${ }^{\circ} \mathrm{C} .{ }^{1} \mathrm{H}$ NMR (400 MHz, DMSO- $\left.d_{6}\right) \delta 10.00(\mathrm{~s}, 1 \mathrm{H}, \mathrm{NH}), 9.83(\mathrm{~s}, 1 \mathrm{H}, \mathrm{NH}), 4.15\left(\mathrm{~s}, 2 \mathrm{H}, \mathrm{CH}_{2}\right), 3.01(\mathrm{~s}, 3 \mathrm{H}$, $\left.\mathrm{CH}_{3}\right), 1.86\left(\mathrm{~s}, 3 \mathrm{H}, \mathrm{CH}_{3}\right) ;{ }^{13} \mathrm{C} \mathrm{NMR}\left(100 \mathrm{MHz}, \mathrm{DMSO}-d_{6}\right) \delta 151.4,146.0,53.9,41.1$, 20.1. ESI-HRMS(m/z): Calcd. for $\mathrm{C}_{5} \mathrm{H}_{11} \mathrm{~N}_{4} \mathrm{O}_{3} \mathrm{~S}[\mathrm{M}+\mathrm{H}]^{+}$207.0546; found 207.0548.

Compounds I-2-I-33 were synthesized using the similar procedure as compound I-1. 
White solid, yield 96\%, Mp 206-208 ${ }^{\circ} \mathrm{C} .{ }^{1} \mathrm{H}$ NMR (300 MHz, DMSO- $\left.d_{6}\right) \delta 9.98(\mathrm{~s}, 1 \mathrm{H}$, $\mathrm{NH}), 9.79(\mathrm{~s}, 1 \mathrm{H}, \mathrm{NH}), 4.15\left(\mathrm{~s}, 2 \mathrm{H}, \mathrm{CH}_{2}\right), 3.10$ (q, $\left.J=7.2 \mathrm{~Hz}, 2 \mathrm{H}, \mathbf{C H}_{2} \mathrm{CH}_{3}\right), 1.85$ (s, $\left.3 \mathrm{H}, \mathrm{CH}_{3}\right), 1.27\left(\mathrm{t}, J=7.2 \mathrm{~Hz}, 3 \mathrm{H}, \mathrm{CH}_{2} \mathbf{C H}_{3}\right) ;{ }^{13} \mathrm{C}$ NMR $\left(100 \mathrm{MHz}, \mathrm{DMSO}-d_{6}\right) \delta 151.2$, 145.7, 53.8, 46.7, 19.8, 7.9. ESI-HRMS(m/z): Calcd. for $\mathrm{C}_{6} \mathrm{H}_{13} \mathrm{~N}_{4} \mathrm{O}_{3} \mathrm{~S}[\mathrm{M}+\mathrm{H}]^{+}$ 221.0703; found 221.0701.

N-(6-Methyl-3-oxo-2,3-dihydro-1,2,4-triazin-4(5H)-yl)propane-1-sulfonamide (I3). White solid, yield 93\%, Mp 177-178 ${ }^{\circ} \mathrm{C} .{ }^{1} \mathrm{H}$ NMR (300 MHz, DMSO- $\left.d_{6}\right) \delta 9.98(\mathrm{~s}$, 1H, NH), $9.81(\mathrm{~s}, 1 \mathrm{H}, \mathrm{NH}), 4.15\left(\mathrm{~s}, 2 \mathrm{H}, \mathrm{CH}_{2}\right), 3.07\left(\mathrm{t}, J=7.5 \mathrm{~Hz}, 2 \mathrm{H}, \mathbf{C H}_{2} \mathrm{CH}_{2} \mathrm{CH}_{3}\right)$, $1.85\left(\mathrm{~s}, 3 \mathrm{H}, \mathrm{CH}_{3}\right), 1.73-1.80\left(\mathrm{~m}, 2 \mathrm{H}, \mathrm{CH}_{2} \mathbf{C H}_{2} \mathrm{CH}_{3}\right), 0.95(\mathrm{t}, J=7.5 \mathrm{~Hz}, 3 \mathrm{H}$, $\left.\mathrm{CH}_{2} \mathrm{CH}_{2} \mathbf{C H}_{3}\right) ;{ }^{13} \mathrm{C}$ NMR $\left(100 \mathrm{MHz}, \mathrm{DMSO}-d_{6}\right) \delta 151.2,145.6,53.8,19.8,16.7,12.8$. ESI-HRMS(m/z): Calcd. for $\mathrm{C}_{7} \mathrm{H}_{15} \mathrm{~N}_{4} \mathrm{O}_{3} \mathrm{~S}[\mathrm{M}+\mathrm{H}]^{+}$235.0859; found 235.0856.

N-(6-Methyl-3-oxo-2,3-dihydro-1,2,4-triazin-4(5H)-yl)butane-1-sulfonamide (I-4) White solid, yield 91\%, Mp 167-169 ${ }^{\circ} \mathrm{C} .{ }^{1} \mathrm{H}$ NMR (300 MHz, DMSO- $\left.d_{6}\right) \delta 9.99(\mathrm{~s}, 1 \mathrm{H}$, $\mathrm{NH}), 9.81(\mathrm{~s}, 1 \mathrm{H}, \mathrm{NH}), 4.15\left(\mathrm{~s}, 2 \mathrm{H}, \mathrm{CH}_{2}\right), 3.10\left(\mathrm{t}, J=7.5 \mathrm{~Hz}, 2 \mathrm{H}, \mathbf{C H}_{2} \mathrm{CH}_{2} \mathrm{CH}_{2} \mathrm{CH}_{3}\right)$, $1.85\left(\mathrm{~s}, 3 \mathrm{H}, \mathrm{CH}_{3}\right), 1.67-1.77\left(\mathrm{~m}, 2 \mathrm{H}, \mathrm{CH}_{2} \mathbf{C H}_{2} \mathrm{CH}_{2} \mathrm{CH}_{3}\right), 1.30-1.40(\mathrm{~m}, 2 \mathrm{H}$, $\left.\mathrm{CH}_{2} \mathrm{CH}_{2} \mathbf{C H}_{2} \mathrm{CH}_{3}\right), 0.87$ (t, $\left.J=7.2 \mathrm{~Hz}, 3 \mathrm{H}, \mathrm{CH}_{2} \mathrm{CH}_{2} \mathrm{CH}_{2} \mathbf{C H}_{3}\right) ;{ }^{13} \mathrm{C}$ NMR (100 MHz, DMSO- $\left.d_{6}\right) \delta 151.2,145.7,53.8,51.9,24.9,20.9,19.8,13.5$. ESI-HRMS(m/z): Calcd. for $\mathrm{C}_{8} \mathrm{H}_{17} \mathrm{~N}_{4} \mathrm{O}_{3} \mathrm{~S}[\mathrm{M}+\mathrm{H}]^{+}$249.1016; found 249.1018.

N-(6-Methyl-3-oxo-2,3-dihydro-1,2,4-triazin-4(5H)-yl)cyclopropanesulfonamide (I-5). White solid, yield 94\%, Mp $146-148{ }^{\circ} \mathrm{C} .{ }^{1} \mathrm{H}$ NMR (400 MHz, DMSO- $\left.d_{6}\right) \delta 9.96$ (s, 1H, NH), $9.79(\mathrm{~s}, 1 \mathrm{H}, \mathrm{NH}), 4.13\left(\mathrm{~s}, 2 \mathrm{H}, \mathrm{CH}_{2}\right), 2.57-2.63\left(\mathrm{~m}, 1 \mathrm{H}, \mathrm{CH}\left(\mathrm{CH}_{2}\right)_{2}\right), 1.86$ $\left(\mathrm{s}, 3 \mathrm{H}, \mathrm{CH}_{3}\right), 0.93-0.98\left(\mathrm{~m}, 4 \mathrm{H}, \mathrm{CH}\left(\mathbf{C H}_{2}\right)_{2}\right) ;{ }^{13} \mathrm{C} \mathrm{NMR}\left(100 \mathrm{MHz}, \mathrm{DMSO}-d_{6}\right) \delta 151.3$, 
145.6, 53.3, 29.9, 19.8, 5.4. ESI-HRMS(m/z): Calcd. for $\mathrm{C}_{7} \mathrm{H}_{13} \mathrm{~N}_{4} \mathrm{O}_{3} \mathrm{~S}[\mathrm{M}+\mathrm{H}]^{+}$ 233.0703; found 233.0703.

N-(6-Methyl-3-oxo-2,3-dihydro-1,2,4-triazin-4(5H)-yl)cyclohexanesulfonamide

(I-6). White solid, yield 83\%, Mp 166-168 ${ }^{\circ} \mathrm{C} .{ }^{1} \mathrm{H}$ NMR (400 MHz, DMSO- $\left.d_{6}\right) \delta 9.95$ (s, 1H, NH), $9.73(\mathrm{~s}, 1 \mathrm{H}, \mathrm{NH}), 4.14\left(\mathrm{~s}, 2 \mathrm{H}, \mathrm{CH}_{2}\right), 2.99-3.06(\mathrm{~m}, 1 \mathrm{H}), 1.85\left(\mathrm{~s}, 3 \mathrm{H}, \mathrm{CH}_{3}\right)$, 1.76-1.79 (m, 2H), 1.28-1.37 (m, 3H), 1.07-1.25 (m, 5H); ${ }^{13} \mathrm{C}$ NMR (100 MHz, DMSO- $\left.d_{6}\right) \delta 151.3,145.6,59.8,53.9,25.8,24.8,24.7,19.8$. ESI-HRMS(m/z): Calcd. for $\mathrm{C}_{10} \mathrm{H}_{18} \mathrm{~N}_{4} \mathrm{O}_{3} \mathrm{~S}[\mathrm{M}+\mathrm{H}]^{+}$275.1172; found 275.1170.

N-(6-Methyl-3-oxo-2,3-dihydro-1,2,4-triazin-4(5H)-yl)-1-

phenylmethanesulfonamide (I-7). White solid, yield 88\%, Mp $189-190{ }^{\circ} \mathrm{C} .{ }^{1} \mathrm{H}$ NMR $\left(300 \mathrm{MHz}, \mathrm{DMSO}-d_{6}\right) \delta 10.04(\mathrm{~s}, 1 \mathrm{H}, \mathrm{NH}), 9.86(\mathrm{~s}, 1 \mathrm{H}, \mathrm{NH}), 7.46-7.50(\mathrm{~m}, 2 \mathrm{H}, \mathrm{Ar}-$ H), 7.33-7.40 (m, 3H, Ar-H), $4.46\left(\mathrm{~s}, 2 \mathrm{H}, \mathrm{CH}_{2}\right), 4.13\left(\mathrm{~s}, 2 \mathrm{H}, \mathrm{CH}_{2}\right), 1.86\left(\mathrm{~s}, 3 \mathrm{H}, \mathrm{CH}_{3}\right)$; ${ }^{13} \mathrm{C}$ NMR $\left(100 \mathrm{MHz}, \mathrm{DMSO}-d_{6}\right) \delta 151.3,145.6,131.2,129.4,128.4,128.2,58.7,53.8$, 19.8. ESI-HRMS(m/z): Calcd. for $\mathrm{C}_{11} \mathrm{H}_{15} \mathrm{~N}_{4} \mathrm{O}_{3} \mathrm{~S}[\mathrm{M}+\mathrm{H}]^{+}$283.0859; found 283.0860. N-(6-Methyl-3-oxo-2,3-dihydro-1,2,4-triazin-4(5H)-yl)benzenesulfonamide (I-8). White solid, yield 89\%, Mp 206-208 ${ }^{\circ} \mathrm{C} .{ }^{1} \mathrm{H}$ NMR (400 MHz, DMSO- $\left.d_{6}\right) \delta 10.23$ (s, 1H, NH), 9.81 (s, 1H, NH), 7.80-7.82 (m, 2H, Ar-H), 7.64-7.67 (m, 1H, Ar-H), 7.54$7.58(\mathrm{~m}, 2 \mathrm{H}, \mathrm{Ar}-\mathrm{H}), 4.05\left(\mathrm{~s}, 2 \mathrm{H}, \mathrm{CH}_{2}\right), 1.83\left(\mathrm{~s}, 3 \mathrm{H}, \mathrm{CH}_{3}\right) ;{ }^{13} \mathrm{C}$ NMR $(100 \mathrm{MHz}, \mathrm{DMSO}-$ $\left.d_{6}\right) \delta 150.7,145.6,138.8,133.2,128.9,127.7,52.7,19.9$. ESI-HRMS(m/z): Calcd. for $\mathrm{C}_{10} \mathrm{H}_{13} \mathrm{~N}_{4} \mathrm{O}_{3} \mathrm{~S}[\mathrm{M}+\mathrm{H}]^{+}$269.0703; found 269.0704.

2-Methyl-N-(6-methyl-3-oxo-2,3-dihydro-1,2,4-triazin-4(5H)-

yl)benzenesulfonamide (I-9). White solid, yield 79\%, Mp 240-241 ${ }^{\circ} \mathrm{C} .{ }^{1} \mathrm{H}$ NMR (400 
$\left.\mathrm{MHz}, \mathrm{DMSO}-d_{6}\right) \delta 10.15(\mathrm{~s}, 1 \mathrm{H}, \mathrm{NH}), 9.76(\mathrm{~s}, 1 \mathrm{H}, \mathrm{NH}), 7.84(\mathrm{~d}, J=7.6 \mathrm{~Hz}, 1 \mathrm{H}, \mathrm{Ar}-$ H), $7.51(\mathrm{t}, J=6.8 \mathrm{~Hz}, 1 \mathrm{H}, \mathrm{Ar}-\mathrm{H}), 7.31-7.37(\mathrm{~m}, 2 \mathrm{H}, \mathrm{Ar}-\mathrm{H}), 4.03\left(\mathrm{~s}, 2 \mathrm{H}, \mathrm{CH}_{2}\right), 2.65$ $\left(\mathrm{s}, 3 \mathrm{H}, \mathrm{CH}_{3}\right), 1.81\left(\mathrm{~s}, 3 \mathrm{H}, \mathrm{CH}_{3}\right) ;{ }^{13} \mathrm{C} \mathrm{NMR}\left(100 \mathrm{MHz}, \mathrm{DMSO}-d_{6}\right) \delta 150.9,145.6,138.4$, 136.9, 133.2, 132.2, 129.6, 125.8, 52.7, 20.2, 19.8. ESI-HRMS(m/z): Calcd. for $\mathrm{C}_{11} \mathrm{H}_{15} \mathrm{~N}_{4} \mathrm{O}_{3} \mathrm{~S}[\mathrm{M}+\mathrm{H}]^{+}$283.0859; found 283.0859.

3-Methyl-N-(6-methyl-3-oxo-2,3-dihydro-1,2,4-triazin-4(5H)-

yl)benzenesulfonamide (I-10). White solid, yield 92\%, Mp 150-151 ${ }^{\circ} \mathrm{C} .{ }^{1} \mathrm{H}$ NMR (400 $\left.\mathrm{MHz}, \mathrm{DMSO}-d_{6}\right) \delta 10.16(\mathrm{~s}, 1 \mathrm{H}, \mathrm{NH}), 9.79(\mathrm{~s}, 1 \mathrm{H}, \mathrm{NH}), 7.60-7.63(\mathrm{~m}, 2 \mathrm{H}, \mathrm{Ar}-\mathrm{H})$, 7.42-7.46(m, 2H, Ar-H), $4.03\left(\mathrm{~s}, 2 \mathrm{H}, \mathrm{CH}_{2}\right), 2.37\left(\mathrm{~s}, 3 \mathrm{H}, \mathrm{CH}_{3}\right), 1.82\left(\mathrm{~s}, 3 \mathrm{H}, \mathrm{CH}_{3}\right) ;{ }^{13} \mathrm{C}$ NMR (100 MHz, DMSO- $\left.d_{6}\right) \delta 150.7,145.6,138.8,138.5,133.8,128.7,127.8,124.9$, 52.7, 20.8, 19.8. ESI-HRMS(m/z): Calcd. for $\mathrm{C}_{11} \mathrm{H}_{15} \mathrm{~N}_{4} \mathrm{O}_{3} \mathrm{~S}[\mathrm{M}+\mathrm{H}]^{+}$283.0859; found 283.0858 .

4-Methyl-N-(6-methyl-3-oxo-2,3-dihydro-1,2,4-triazin-4(5H)-

yl)benzenesulfonamide (I-11). White solid, yield 98\%, Mp 199-200 ${ }^{\circ} \mathrm{C} .{ }^{1} \mathrm{H}$ NMR $(300$ MHz, DMSO- $\left.d_{6}\right) \delta 10.10(\mathrm{~s}, 1 \mathrm{H}, \mathrm{NH}), 9.79(\mathrm{~s}, 1 \mathrm{H}, \mathrm{NH}), 7.69(\mathrm{~d}, J=8.4 \mathrm{~Hz}, 2 \mathrm{H}, \mathrm{Ar}-$ H), $7.36(\mathrm{~d}, J=8.1 \mathrm{~Hz}, 2 \mathrm{H}, \mathrm{Ar}-\mathrm{H}), 4.05\left(\mathrm{~s}, 2 \mathrm{H}, \mathrm{CH}_{2}\right), 2.38\left(\mathrm{~s}, 3 \mathrm{H}, \mathrm{CH}_{3}\right), 1.83(\mathrm{~s}, 3 \mathrm{H}$, $\left.\mathrm{CH}_{3}\right) ;{ }^{13} \mathrm{C} \mathrm{NMR}\left(100 \mathrm{MHz}, \mathrm{DMSO}-d_{6}\right) \delta 150.7,145.7,143.5,135.9,129.4,127.8,52.7$, 21.1, 19.9. ESI-HRMS(m/z): Calcd. for $\mathrm{C}_{11} \mathrm{H}_{15} \mathrm{~N}_{4} \mathrm{O}_{3} \mathrm{~S}[\mathrm{M}+\mathrm{H}]^{+}$283.0859; found 283.0863.

2,4,6-Trimethyl-N-(6-methyl-3-oxo-2,3-dihydro-1,2,4-triazin-4(5H)-

yl)benzenesulfonamide (I-12). White solid, yield 87\%, Mp 224-226 ${ }^{\circ} \mathrm{C} .{ }^{1} \mathrm{H}$ NMR (400 MHz, DMSO- $\left.d_{6}\right) \delta 9.91(\mathrm{~s}, 1 \mathrm{H}, \mathrm{NH}), 9.80(\mathrm{~s}, 1 \mathrm{H}, \mathrm{NH}), 6.99(\mathrm{~s}, 2 \mathrm{H}, \mathrm{Ar}-\mathrm{H}), 4.01(\mathrm{~s}, 2 \mathrm{H}$, 
$\left.\mathrm{CH}_{2}\right), 2.54\left(\mathrm{~s}, 3 \mathrm{H}, \mathrm{CH}_{3}\right), 2.24\left(\mathrm{~s}, 3 \mathrm{H}, \mathrm{CH}_{3}\right), 1.82\left(\mathrm{~s}, 3 \mathrm{H}, \mathrm{CH}_{3}\right) ;{ }^{13} \mathrm{C} \mathrm{NMR}(100 \mathrm{MHz}$, DMSO- $\left.d_{6}\right) \delta 151.2,145.9,142.2,139.8,133.0,131.4,52.3,22.7,20.5,19.9$. ESIHRMS(m/z): Calcd. for $\mathrm{C}_{13} \mathrm{H}_{19} \mathrm{~N}_{4} \mathrm{O}_{3} \mathrm{~S}[\mathrm{M}+\mathrm{H}]^{+}$311.1172;found 311.1172.

4-Methoxy-N-(6-methyl-3-oxo-2,3-dihydro-1,2,4-triazin-4(5H)-

yl)benzenesulfonamide (I-13). White solid, yield 88\%, Mp 245-246 ${ }^{\circ} \mathrm{C} .{ }^{1} \mathrm{H}$ NMR (400 MHz, DMSO- $\left.d_{6}\right) \delta 9.99(\mathrm{~s}, 1 \mathrm{H}, \mathrm{NH}), 9.78(\mathrm{~s}, 1 \mathrm{H}, \mathrm{NH}), 7.73(\mathrm{~d}, J=8.8 \mathrm{~Hz}, 2 \mathrm{H}, \mathrm{Ar}-\mathrm{H})$, $7.06(\mathrm{~d}, J=8.8 \mathrm{~Hz}, 2 \mathrm{H}, \mathrm{Ar}-\mathrm{H}), 4.04\left(\mathrm{~s}, 2 \mathrm{H}, \mathrm{CH}_{2}\right), 3.82\left(\mathrm{~s}, 3 \mathrm{H}, \mathrm{OCH}_{3}\right), 1.83(\mathrm{~s}, 3 \mathrm{H}$, $\left.\mathrm{CH}_{3}\right) ;{ }^{13} \mathrm{C} \mathrm{NMR}\left(100 \mathrm{MHz}, \mathrm{DMSO}-d_{6}\right) \delta 162.8,150.7,145.6,130.1,130.0,114.1,55.7$, 52.6, 19.9. ESI-HRMS(m/z): Calcd. for $\mathrm{C}_{11} \mathrm{H}_{15} \mathrm{~N}_{4} \mathrm{O}_{4} \mathrm{~S}[\mathrm{M}+\mathrm{H}]^{+}$299.0809; found 299.0807.

4-(Tert-butyl)-N-(6-methyl-3-oxo-2,3-dihydro-1,2,4-triazin-4(5H)-

yl)benzenesulfonamide (I-14). White solid, yield 79\%, Mp 240-241 ${ }^{\circ} \mathrm{C} .{ }^{1} \mathrm{H}$ NMR (400 $\left.\mathrm{MHz}, \mathrm{DMSO}-d_{6}\right) \delta 10.08(\mathrm{~s}, 1 \mathrm{H}, \mathrm{NH}), 9.85(\mathrm{~s}, 1 \mathrm{H}, \mathrm{NH}), 7.74(\mathrm{~d}, J=8.0 \mathrm{~Hz}, 2 \mathrm{H}, \mathrm{Ar}-$ H), $7.59(\mathrm{~d}, J=8.0 \mathrm{~Hz}, 2 \mathrm{H}, \mathrm{Ar}-\mathrm{H}), 3.98\left(\mathrm{~s}, 2 \mathrm{H}, \mathrm{CH}_{2}\right), 1.80\left(\mathrm{~s}, 3 \mathrm{H}, \mathrm{CH}_{3}\right), 1.29(\mathrm{~s}, 9 \mathrm{H}$, $\left.\mathrm{C}\left(\mathrm{CH}_{3}\right)_{3}\right) ;{ }^{13} \mathrm{C}$ NMR $\left(100 \mathrm{MHz}, \mathrm{DMSO}-d_{6}\right) \delta 156.2,150.9,145.6,136.2,127.6,125.8$, 52.6, 34.9, 30.8, 19.8. ESI-HRMS(m/z): Calcd. for $\mathrm{C}_{14} \mathrm{H}_{21} \mathrm{~N}_{4} \mathrm{O}_{3} \mathrm{~S}[\mathrm{M}+\mathrm{H}]^{+}$325.1329; found 325.1329 .

2-Fluoro-N-(6-methyl-3-oxo-2,3-dihydro-1,2,4-triazin-4(5H)-

yl)benzenesulfonamide (I-15). White solid, yield 93\%, Mp 206-208 ${ }^{\circ} \mathrm{C} .{ }^{1} \mathrm{H}$ NMR (400 MHz, DMSO- $\left.d_{6}\right) \delta 10.49(\mathrm{~s}, 1 \mathrm{H}, \mathrm{NH}), 9.80(\mathrm{~s}, 1 \mathrm{H}, \mathrm{NH}), 7.79(\mathrm{t}, J=6.8 \mathrm{~Hz}, 1 \mathrm{H}, \mathrm{Ar}-$ H), 7.67-7.72 (m, 1H, Ar-H), 7.39 (t, $J=9.6 \mathrm{~Hz}, 1 \mathrm{H}, \mathrm{Ar}-\mathrm{H}), 7.33$ (t, $J=7.6 \mathrm{~Hz}, 1 \mathrm{H}$, $\mathrm{Ar}-\mathrm{H}), 4.13\left(\mathrm{~s}, 2 \mathrm{H}, \mathrm{CH}_{2}\right), 1.85\left(\mathrm{~s}, 3 \mathrm{H}, \mathrm{CH}_{3}\right) ;{ }^{13} \mathrm{C}$ NMR $\left(100 \mathrm{MHz}, \mathrm{DMSO}-d_{6}\right) \delta 159.2$ 
(d, $J=254.0 \mathrm{~Hz}), 150.6,145.5,135.9(\mathrm{~d}, J=8.7 \mathrm{~Hz}), 130.2,127.1(\mathrm{~d}, J=14 \mathrm{~Hz}), 124.3$ (d, $J=3.2 \mathrm{~Hz}), 117.0(\mathrm{~d}, J=20.9 \mathrm{~Hz}), 53.2,19.8$. ESI-HRMS(m/z): Calcd. for $\mathrm{C}_{10} \mathrm{H}_{12} \mathrm{FN}_{4} \mathrm{O}_{3} \mathrm{~S}[\mathrm{M}+\mathrm{H}]^{+}$287.0609; found 287.0609.

3-Fluoro-N-(6-methyl-3-oxo-2,3-dihydro-1,2,4-triazin-4(5H)-

yl)benzenesulfonamide (I-16). White solid, yield 91\%, Mp 180-181 ${ }^{\circ} \mathrm{C} .{ }^{1} \mathrm{H}$ NMR (400 MHz, DMSO- $\left.d_{6}\right) \delta 10.45(\mathrm{~s}, 1 \mathrm{H}, \mathrm{NH}), 9.83(\mathrm{~s}, 1 \mathrm{H}, \mathrm{NH}), 7.66(\mathrm{~d}, J=8.0 \mathrm{~Hz}, 1 \mathrm{H}, \mathrm{Ar}-$ H), 7.59-7.63 (m, 2H, Ar-H), $7.53(\mathrm{t}, J=8.4 \mathrm{~Hz}, 1 \mathrm{H}, \mathrm{Ar}-\mathrm{H}), 4.11\left(\mathrm{~s}, 2 \mathrm{H}, \mathrm{CH}_{2}\right), 1.85$ (s, 3H, $\left.\mathrm{CH}_{3}\right) ;{ }^{13} \mathrm{C}$ NMR $\left(100 \mathrm{MHz}\right.$, DMSO- $\left.d_{6}\right) \delta 161.5(\mathrm{~d}, J=246.1 \mathrm{~Hz}), 150.5,145.6$, $141.0(\mathrm{~d}, J=7.0 \mathrm{~Hz}), 131.1(\mathrm{~d}, J=7.7 \mathrm{~Hz}), 124.0(\mathrm{~d}, J=2.7 \mathrm{~Hz}), 120.3(\mathrm{~d}, J=20.9$ Hz), $114.7(\mathrm{~d}, J=24.3 \mathrm{~Hz}), 53.0,19.8$. ESI-HRMS(m/z): Calcd. for $\mathrm{C}_{10} \mathrm{H}_{12} \mathrm{FN}_{4} \mathrm{O}_{3} \mathrm{~S}$ $[\mathrm{M}+\mathrm{H}]^{+}$287.0609; found 287.0605.

4-Fluoro-N-(6-methyl-3-oxo-2,3-dihydro-1,2,4-triazin-4(5H)-

yl)benzenesulfonamide (I-17). White solid, yield 95\%, Mp 216-217 ${ }^{\circ} \mathrm{C} .{ }^{1} \mathrm{H}$ NMR (400 MHz, DMSO- $\left.d_{6}\right) \delta 10.29(\mathrm{~s}, 1 \mathrm{H}, \mathrm{NH}), 9.81(\mathrm{~s}, 1 \mathrm{H}, \mathrm{NH}), 7.85-7.89(\mathrm{~m}, 2 \mathrm{H}, \mathrm{Ar}-\mathrm{H}), 7.40$ (t, $J=8.8 \mathrm{~Hz}, 2 \mathrm{H}, \mathrm{Ar}-\mathrm{H}), 4.11\left(\mathrm{~s}, 2 \mathrm{H}, \mathrm{CH}_{2}\right), 1.85\left(\mathrm{~s}, 3 \mathrm{H}, \mathrm{CH}_{3}\right) ;{ }^{13} \mathrm{C} \mathrm{NMR}(100 \mathrm{MHz}$, DMSO- $\left.d_{6}\right) \delta 164.7(\mathrm{~d}, J=249.9 \mathrm{~Hz}), 150.6,145.7,135.1(\mathrm{~d}, J=2.7 \mathrm{~Hz}), 130.9(\mathrm{~d}, J=$ 9.7 Hz), $116.0(\mathrm{~d}, J=22.6 \mathrm{~Hz}), 52.9$, 19.9. ESI-HRMS(m/z): Calcd. for $\mathrm{C}_{10} \mathrm{H}_{12} \mathrm{FN}_{4} \mathrm{O}_{3} \mathrm{~S}$ $[\mathrm{M}+\mathrm{H}]^{+}$287.0609; found 287.0606.

2,4-Difluoro-N-(6-methyl-3-oxo-2,3-dihydro-1,2,4-triazin-4(5H)-

yl)benzenesulfonamide (I-18). White solid, yield 97\%, Mp 211-213 ${ }^{\circ} \mathrm{C} .{ }^{1} \mathrm{H}$ NMR (400 MHz, DMSO- $\left.d_{6}\right) \delta 10.59(\mathrm{~s}, 1 \mathrm{H}, \mathrm{NH}), 9.82(\mathrm{~s}, 1 \mathrm{H}, \mathrm{NH}), 7.81-7.87(\mathrm{~m}, 1 \mathrm{H}, \mathrm{Ar}-\mathrm{H}), 7.50$ (t, $J=10.0 \mathrm{~Hz}, 1 \mathrm{H}, \mathrm{Ar}-\mathrm{H}), 7.21(\mathrm{t}, J=8.4 \mathrm{~Hz}, 1 \mathrm{H}, \mathrm{Ar}-\mathrm{H}), 4.14\left(\mathrm{~s}, 2 \mathrm{H}, \mathrm{CH}_{2}\right), 1.85(\mathrm{~s}$, 
$\left.3 \mathrm{H}, \mathrm{CH}_{3}\right) ;{ }^{13} \mathrm{C} \mathrm{NMR}\left(100 \mathrm{MHz}, \mathrm{DMSO}-d_{6}\right) \delta 165.5(\mathrm{dd}, J=253.3 \mathrm{~Hz}, 12.5 \mathrm{~Hz}), 160.2$ $(\mathrm{dd}, J=257.4 \mathrm{~Hz}, 14.1 \mathrm{~Hz}), 150.6,145.5,132.4(\mathrm{~d}, J=10.8 \mathrm{~Hz}), 123.9(\mathrm{q}, J=4.4 \mathrm{~Hz})$, $111.7(\mathrm{q}, J=4.1 \mathrm{~Hz}), 105.7$ (t, $J=25.7 \mathrm{~Hz}), 53.3,19.8$. ESI-HRMS(m/z): Calcd. for $\mathrm{C}_{10} \mathrm{H}_{11} \mathrm{~F}_{2} \mathrm{~N}_{4} \mathrm{O}_{3} \mathrm{~S}[\mathrm{M}+\mathrm{H}]^{+}$305.0514; found 305.0511.

2,3,4-Trifluoro-N-(6-methyl-3-oxo-2,3-dihydro-1,2,4-triazin-4(5H)-

yl)benzenesulfonamide (I-19). White solid, yield 94\%, Mp 214-216 ${ }^{\circ} \mathrm{C} .{ }^{1} \mathrm{H}$ NMR (400 MHz, DMSO- $\left.d_{6}\right) \delta 10.88(\mathrm{~s}, 1 \mathrm{H}, \mathrm{NH}), 9.89(\mathrm{~s}, 1 \mathrm{H}, \mathrm{NH}), 7.66-7.71(\mathrm{~m}, 1 \mathrm{H}, \mathrm{Ar}-\mathrm{H})$, 7.45-7.51 (m, 1H, Ar-H), $4.18\left(\mathrm{~s}, 2 \mathrm{H}, \mathrm{CH}_{2}\right), 1.87\left(\mathrm{~s}, 3 \mathrm{H}, \mathrm{CH}_{3}\right) ;{ }^{13} \mathrm{C} \mathrm{NMR}(100 \mathrm{MHz}$, DMSO- $\left.d_{6}\right) \delta 153.6(\mathrm{q}, J=252.6 \mathrm{~Hz}, 9.5 \mathrm{~Hz}), 150.7,148.9(\mathrm{dd}, J=257.2 \mathrm{~Hz}, 8.1 \mathrm{~Hz})$, $145.7,140.8(\mathrm{t}, J=15.6 \mathrm{~Hz}), 139.5(\mathrm{dt}, J=250.3 \mathrm{~Hz}, 15.3 \mathrm{~Hz}), 125.3(\mathrm{~m}), 112.6(\mathrm{q}, J$ $=2.6 \mathrm{~Hz}), 53.4$, 19.8. ESI-HRMS(m/z): Calcd. for $\mathrm{C}_{10} \mathrm{H}_{10} \mathrm{~F}_{3} \mathrm{~N}_{4} \mathrm{O}_{3} \mathrm{~S}[\mathrm{M}+\mathrm{H}]^{+}$323.0420; found 323.0414 .

4-Chloro-N-(6-methyl-3-oxo-2,3-dihydro-1,2,4-triazin-4(5H)-

yl)benzenesulfonamide (I-20). White solid, yield 98\%, Mp 215-216 ${ }^{\circ} \mathrm{C} .{ }^{1} \mathrm{H}$ NMR $(400$ $\left.\mathrm{MHz}, \mathrm{DMSO}-d_{6}\right) \delta 10.38(\mathrm{~s}, 1 \mathrm{H}, \mathrm{NH}), 9.82(\mathrm{~s}, 1 \mathrm{H}, \mathrm{NH}), 7.80(\mathrm{~d}, J=8.4 \mathrm{~Hz}, 2 \mathrm{H}, \mathrm{Ar}-$ $\mathrm{H}), 7.63(\mathrm{~d}, J=8.4 \mathrm{~Hz}, 2 \mathrm{H}, \mathrm{Ar}-\mathrm{H}), 4.12\left(\mathrm{~s}, 2 \mathrm{H}, \mathrm{CH}_{2}\right), 1.85\left(\mathrm{~s}, 3 \mathrm{H}, \mathrm{CH}_{3}\right) ;{ }^{13} \mathrm{C} \mathrm{NMR}$ $\left(100 \mathrm{MHz}, \mathrm{DMSO}-d_{6}\right) \delta 150.5,145.6,138.1,137.7,129.7,129.0,53.0,19.9$. ESI$\operatorname{HRMS}(\mathrm{m} / \mathrm{z})$ : Calcd. for $\mathrm{C}_{10} \mathrm{H}_{12} \mathrm{ClN}_{4} \mathrm{O}_{3} \mathrm{~S}[\mathrm{M}+\mathrm{H}]^{+}$303.0313; found 303.0316. 4-Bromo-N-(6-methyl-3-oxo-2,3-dihydro-1,2,4-triazin-4(5H)-

yl)benzenesulfonamide (I-21). White solid, yield 95\%, Mp 230-232 ${ }^{\circ} \mathrm{C} .{ }^{1} \mathrm{H}$ NMR (400 $\left.\mathrm{MHz}, \mathrm{DMSO}-d_{6}\right) \delta 10.40(\mathrm{~s}, 1 \mathrm{H}, \mathrm{NH}), 9.84(\mathrm{~s}, 1 \mathrm{H}, \mathrm{NH}), 7.79(\mathrm{~d}, J=8.4 \mathrm{~Hz}, 2 \mathrm{H}, \mathrm{Ar}-$ $\mathrm{H}), 7.73(\mathrm{~d}, J=8.4 \mathrm{~Hz}, 2 \mathrm{H}, \mathrm{Ar}-\mathrm{H}), 4.13\left(\mathrm{~s}, 2 \mathrm{H}, \mathrm{CH}_{2}\right), 1.86\left(\mathrm{~s}, 3 \mathrm{H}, \mathrm{CH}_{3}\right) ;{ }^{13} \mathrm{C} \mathrm{NMR}$ 
$\left(100 \mathrm{MHz}, \mathrm{DMSO}-d_{6}\right) \delta 150.6,145.7,138.1,132.0,129.8,127.2,53.0,19.9$. ESIHRMS(m/z): Calcd. for $\mathrm{C}_{10} \mathrm{H}_{12} \mathrm{BrN}_{4} \mathrm{O}_{3} \mathrm{~S}[\mathrm{M}+\mathrm{H}]^{+} 346.9808$; found 346.9804 .

4-Iodo-N-(6-methyl-3-oxo-2,3-dihydro-1,2,4-triazin-4(5H)-

yl)benzenesulfonamide (I-22). White solid, yield 91\%, Mp 241-243 ${ }^{\circ} \mathrm{C} .{ }^{1} \mathrm{H}$ NMR (400 $\left.\mathrm{MHz}, \mathrm{DMSO}-d_{6}\right) \delta 10.36(\mathrm{~s}, 1 \mathrm{H}, \mathrm{NH}), 9.84(\mathrm{~s}, 1 \mathrm{H}, \mathrm{NH}), 7.95(\mathrm{~d}, J=8.4 \mathrm{~Hz}, 2 \mathrm{H}, \mathrm{Ar}-$ $\mathrm{H}), 7.56(\mathrm{~d}, J=8.4 \mathrm{~Hz}, 2 \mathrm{H}, \mathrm{Ar}-\mathrm{H}), 4.11\left(\mathrm{~s}, 2 \mathrm{H}, \mathrm{CH}_{2}\right), 1.85\left(\mathrm{~s}, 3 \mathrm{H}, \mathrm{CH}_{3}\right) ;{ }^{13} \mathrm{C} \mathrm{NMR}$ $\left(100 \mathrm{MHz}, \mathrm{DMSO}-d_{6}\right) \delta 150.5,145.6,138.5,137.7,129.4,101.5,52.9,19.8$. ESI$\operatorname{HRMS}(\mathrm{m} / \mathrm{z})$ : Calcd. for $\mathrm{C}_{10} \mathrm{H}_{12} \mathrm{IN}_{4} \mathrm{O}_{3} \mathrm{~S}[\mathrm{M}+\mathrm{H}]^{+}$394.9669; found 394.9666 .

N-(4-(N-(6-Methyl-3-oxo-2,3-dihydro-1,2,4-triazin-4(5H)-

yl)sulfamoyl)phenyl)acetamide (I-23). White solid, yield 85\%, Mp 255-257 ${ }^{\circ} \mathrm{C} .{ }^{1} \mathrm{H}$ NMR (300 MHz, DMSO- $\left.d_{6}\right) \delta 10.32(\mathrm{~s}, 1 \mathrm{H}, \mathrm{NH}), 10.02(\mathrm{~s}, 1 \mathrm{H}, \mathrm{NH}), 9.77(\mathrm{~s}, 1 \mathrm{H}, \mathrm{NH})$, 7.72 (brs, 4H), $4.05\left(\mathrm{~s}, 2 \mathrm{H}, \mathrm{CH}_{2}\right), 2.08\left(\mathrm{~s}, 3 \mathrm{H}, \mathrm{CH}_{3}\right), 1.83\left(\mathrm{~s}, 3 \mathrm{H}, \mathrm{CH}_{3}\right) ;{ }^{13} \mathrm{C} \mathrm{NMR}(100$ $\left.\mathrm{MHz}, \mathrm{DMSO}-d_{6}\right) \delta 169.1,150.7,145.6,143.5,132.0,129.0,118.2,52.6,24.2,19.9$. ESI-HRMS(m/z): Calcd. for $\mathrm{C}_{12} \mathrm{H}_{16} \mathrm{~N}_{5} \mathrm{O}_{4} \mathrm{~S}[\mathrm{M}+\mathrm{H}]^{+}$326.0918; found 326.0921. 4-Cyano-N-(6-methyl-3-oxo-2,3-dihydro-1,2,4-triazin-4(5H)yl)benzenesulfonamide (I-24). White solid, yield 85\%, Mp 256-257 ${ }^{\circ} \mathrm{C} .{ }^{1} \mathrm{H}$ NMR (400 $\left.\mathrm{MHz}, \mathrm{DMSO}-d_{6}\right) \delta 10.66(\mathrm{~s}, 1 \mathrm{H}, \mathrm{NH}), 9.85(\mathrm{~s}, 1 \mathrm{H}, \mathrm{NH}), 8.06(\mathrm{~d}, J=8.0 \mathrm{~Hz}, 2 \mathrm{H}, \mathrm{Ar}-$ H), $7.97(\mathrm{~d}, J=8.0 \mathrm{~Hz}, 2 \mathrm{H}, \mathrm{Ar}-\mathrm{H}), 4.15\left(\mathrm{~s}, 2 \mathrm{H}, \mathrm{CH}_{2}\right), 1.86\left(\mathrm{~s}, 3 \mathrm{H}, \mathrm{CH}_{3}\right) ;{ }^{13} \mathrm{C} \mathrm{NMR}$ $\left(100 \mathrm{MHz}, \mathrm{DMSO}-d_{6}\right) \delta 150.5,145.7,143.2,132.9,128.5,117.7,115.4,53.2,19.8$. ESI-HRMS(m/z): Calcd. for $\mathrm{C}_{11} \mathrm{H}_{12} \mathrm{IN}_{5} \mathrm{O}_{3} \mathrm{~S}[\mathrm{M}+\mathrm{H}]^{+}$294.0655; found 294.0659.

N-(6-Methyl-3-oxo-2,3-dihydro-1,2,4-triazin-4(5H)-yl)-4-

(trifluoromethyl)benzenesulfonamide (I-25). White solid, yield 94\%, Mp 243-244 ${ }^{\circ} \mathrm{C}$. 
${ }^{1} \mathrm{H}$ NMR $\left(400 \mathrm{MHz}, \mathrm{DMSO}-d_{6}\right) \delta 10.59(\mathrm{~s}, 1 \mathrm{H}, \mathrm{NH}), 9.86(\mathrm{~s}, 1 \mathrm{H}, \mathrm{NH}), 8.02(\mathrm{~d}, J=8.4$ $\mathrm{Hz}, 2 \mathrm{H}, \mathrm{Ar}-\mathrm{H}), 7.95(\mathrm{~d}, J=8.4 \mathrm{~Hz}, 2 \mathrm{H}, \mathrm{Ar}-\mathrm{H}), 4.15\left(\mathrm{~s}, 2 \mathrm{H}, \mathrm{CH}_{2}\right), 1.86\left(\mathrm{~s}, 3 \mathrm{H}, \mathrm{CH}_{3}\right)$; ${ }^{13} \mathrm{C}$ NMR (100 MHz, DMSO- $\left.d_{6}\right) \delta 150.6,145.7,143.0,132.7$ (q, $\left.J=32 \mathrm{~Hz}\right), 128.8$, $126.0(\mathrm{q}, J=4.3 \mathrm{~Hz}), 123.6$ (q, $J=271.2 \mathrm{~Hz}), 53.2,19.9$. ESI-HRMS(m/z): Calcd. for $\mathrm{C}_{11} \mathrm{H}_{12} \mathrm{~F}_{3} \mathrm{~N}_{4} \mathrm{O}_{3} \mathrm{~S}[\mathrm{M}+\mathrm{H}]^{+}$337.0577; found 337.0577.

N-(6-methyl-3-oxo-2,3-dihydro-1,2,4-triazin-4(5H)-yl)-4-

(trifluoromethoxy)benzenesulfonamide (I-26). White solid, yield 90\%, Mp 215-216 ${ }^{\circ} \mathrm{C}$. ${ }^{1} \mathrm{H}$ NMR (400 MHz, DMSO- $\left.d_{6}\right) \delta 10.43(\mathrm{~s}, 1 \mathrm{H}, \mathrm{NH}), 9.85(\mathrm{~s}, 1 \mathrm{H}, \mathrm{NH}), 7.94(\mathrm{~d}, J=8.4$ $\mathrm{Hz}, 2 \mathrm{H}, \mathrm{Ar}-\mathrm{H}), 7.55$ (d, J=8.4 Hz, 2H, Ar-H), $4.12\left(\mathrm{~s}, 2 \mathrm{H}, \mathrm{CH}_{2}\right), 1.85\left(\mathrm{~s}, 3 \mathrm{H}, \mathrm{CH}_{3}\right)$; ${ }^{13} \mathrm{C}$ NMR $\left(100 \mathrm{MHz}, \mathrm{DMSO}-d_{6}\right) \delta 151.4,150.6,145.7,137.9,130.5,120.9,119.9(\mathrm{q}$, $J=257.7 \mathrm{~Hz})$, 53.1, 19.8. ESI-HRMS(m/z): Calcd. for $\mathrm{C}_{11} \mathrm{H}_{12} \mathrm{~F}_{3} \mathrm{~N}_{4} \mathrm{O}_{4} \mathrm{~S}[\mathrm{M}+\mathrm{H}]^{+}$ 353.0526; found 353.0525.

N-(6-Methyl-3-oxo-2,3-dihydro-1,2,4-triazin-4(5H)-yl)thiophene-2-sulfonamide

(I-27). White solid, yield 89\%, Mp $191-193{ }^{\circ} \mathrm{C} .{ }^{1} \mathrm{H}$ NMR (400 MHz, DMSO- $\left.d_{6}\right) \delta$ 10.33 (s, 1H, NH), 9.87 (s, 1H, NH), 7.99 (d, $J=5.2 \mathrm{~Hz}, 1 \mathrm{H}, \mathrm{Ar}-\mathrm{H}), 7.63$ (d, $J=3.6$ $\mathrm{Hz}, 1 \mathrm{H}, \mathrm{Ar}-\mathrm{H}), 7.17(\mathrm{t}, J=4.0 \mathrm{~Hz}, 1 \mathrm{H}, \mathrm{Ar}-\mathrm{H}), 4.04\left(\mathrm{~s}, 2 \mathrm{H}, \mathrm{CH}_{2}\right), 1.84\left(\mathrm{~s}, 3 \mathrm{H}, \mathrm{CH}_{3}\right)$; ${ }^{13} \mathrm{C}$ NMR (100 MHz, DMSO- $\left.d_{6}\right) \delta 150.8,145.7,138.7,134.5,133.7,127.7,52.6,20.0$. ESI-HRMS(m/z): Calcd. for $\mathrm{C}_{8} \mathrm{H}_{11} \mathrm{~N}_{4} \mathrm{O}_{3} \mathrm{~S}_{2}[\mathrm{M}+\mathrm{H}]^{+}$275.0267;found 275.0262.

N-(6-Methyl-3-oxo-2,3-dihydro-1,2,4-triazin-4(5H)-yl)thiophene-3-sulfonamide

(I-28). White solid, yield $82 \%$, Mp $214-215{ }^{\circ} \mathrm{C} .{ }^{1} \mathrm{H}$ NMR (400 MHz, DMSO- $\left.d_{6}\right) \delta$ $10.14(\mathrm{~s}, 1 \mathrm{H}, \mathrm{NH}), 9.85(\mathrm{~s}, 1 \mathrm{H}, \mathrm{NH}), 8.25(\mathrm{~s}, 1 \mathrm{H}, \mathrm{Het}-\mathrm{H}), 7.71(\mathrm{~d}, J=2.4 \mathrm{~Hz}, 1 \mathrm{H}$, Het-H), $7.31\left(\mathrm{~d}, J=4.8 \mathrm{~Hz}, 1 \mathrm{H}\right.$, Het-H), $4.03\left(\mathrm{~s}, 2 \mathrm{H}, \mathrm{CH}_{2}\right), 1.83\left(\mathrm{~s}, 3 \mathrm{H}, \mathrm{CH}_{3}\right) ;{ }^{13} \mathrm{C} \mathrm{NMR}$ 
$\left(100 \mathrm{MHz}, \mathrm{DMSO}-d_{6}\right) \delta 150.8,145.6,141.0,138.4,132.7,128.5,126.1,52.6,19.9$. ESI-HRMS(m/z): Calcd. for $\mathrm{C}_{8} \mathrm{H}_{10} \mathrm{~N}_{4} \mathrm{O}_{3} \mathrm{~S}_{2}[\mathrm{M}+\mathrm{H}]^{+}$275.0267; found 275.0272.

3,5-Dimethyl-N-(6-methyl-3-oxo-2,3-dihydro-1,2,4-triazin-4(5H)-yl)isoxazole-4sulfonamide (I-29). White oil, yield 75\%. ${ }^{1} \mathrm{H}$ NMR (400 MHz, DMSO- $\left.d_{6}\right) \delta 10.55(\mathrm{~s}$, 1H, NH), $9.96(\mathrm{~s}, 1 \mathrm{H}, \mathrm{NH}), 4.17\left(\mathrm{~s}, 2 \mathrm{H}, \mathrm{CH}_{2}\right), 2.53\left(\mathrm{~s}, 3 \mathrm{H}, \mathrm{CH}_{3}\right), 2.32\left(\mathrm{~s}, 3 \mathrm{H}, \mathrm{CH}_{3}\right)$, $1.88\left(\mathrm{~s}, 3 \mathrm{H}, \mathrm{CH}_{3}\right) ;{ }^{13} \mathrm{C}$ NMR $\left(100 \mathrm{MHz}, \mathrm{DMSO}-d_{6}\right) \delta 173.8,157.9,150.7,146.1,114.7$, 52.9, 19.9, 12.2, 10.5. ESI-HRMS(m/z): Calcd. for $\mathrm{C}_{9} \mathrm{H}_{13} \mathrm{~N}_{5} \mathrm{O}_{4} \mathrm{~S}[\mathrm{M}+\mathrm{H}]^{+}$288.0761; found 288.0759 .

1-Methyl-N-(6-methyl-3-oxo-2,3-dihydro-1,2,4-triazin-4(5H)-yl)-1H-imidazole4-sulfonamide (I-30). Yellow solid, yield 83\%, Mp 188-190 ${ }^{\circ} \mathrm{C} .{ }^{1} \mathrm{H}$ NMR $(400 \mathrm{MHz}$, DMSO- $\left.d_{6}\right) \delta 9.85(\mathrm{~s}, 1 \mathrm{H}, \mathrm{NH}), 9.84(\mathrm{~s}, 1 \mathrm{H}, \mathrm{NH}), 7.88(\mathrm{~s}, 1 \mathrm{H}$, Het-H), $7.85(\mathrm{~s}, 1 \mathrm{H}$, Het$\mathrm{H}), 3.98\left(\mathrm{~s}, 2 \mathrm{H}, \mathrm{CH}_{2}\right), 3.75\left(\mathrm{~s}, 3 \mathrm{H}, \mathrm{CH}_{3}\right), 1.84\left(\mathrm{~s}, 3 \mathrm{H}, \mathrm{CH}_{3}\right) ;{ }^{13} \mathrm{C} \mathrm{NMR}(100 \mathrm{MHz}$, DMSO- $\left.d_{6}\right) \delta 151.2,145.3,139.8,137.9,125.9,52.1,33.5,19.7 . \operatorname{ESI}-H R M S(m / z):$ Calcd. for $\mathrm{C}_{8} \mathrm{H}_{12} \mathrm{~N}_{6} \mathrm{O}_{3} \mathrm{~S}[\mathrm{M}+\mathrm{H}]^{+}$273.0764; found 273.0766.

1,2-Dimethyl-N-(6-methyl-3-oxo-2,3-dihydro-1,2,4-triazin-4(5H)-yl)-1Himidazole-4-sulfonamide (I-31). Yellow solid, yield 87\%, Mp 180-182 ${ }^{\circ} \mathrm{C} .{ }^{1} \mathrm{H}$ NMR (400 MHz, DMSO- $\left.d_{6}\right) \delta 10.55(\mathrm{~s}, 1 \mathrm{H}, \mathrm{NH}), 9.95$ (s, 1H, NH), 8.14 (s, 1H, Het-H), 4.08 (s, 2H, $\left.\mathrm{CH}_{2}\right), 3.71\left(\mathrm{~s}, 3 \mathrm{H}, \mathrm{CH}_{3}\right), 3.16\left(\mathrm{~s}, 3 \mathrm{H}, \mathrm{CH}_{3}\right), 1.84\left(\mathrm{~s}, 3 \mathrm{H}, \mathrm{CH}_{3}\right) ;{ }^{13} \mathrm{C}$ NMR $(100$ MHz, DMSO- $\left.d_{6}\right) \delta 151.1,147.7,145.7,127.0,120.4,52.9,34.0,19.9,11.4$. ESIHRMS(m/z): Calcd. for $\mathrm{C}_{9} \mathrm{H}_{14} \mathrm{~N}_{6} \mathrm{O}_{3} \mathrm{~S}[\mathrm{M}+\mathrm{H}]^{+}$287.0921; found 287.0927.

N-(6-Methyl-3-oxo-2,3-dihydro-1,2,4-triazin-4(5H)-yl)-2,3-dihydrobenzofuran5-sulfonamide (I-32). White solid, yield 83\%, Mp 263-264 ${ }^{\circ} \mathrm{C} .{ }^{1} \mathrm{H}$ NMR (400 MHz, 
DMSO- $\left.d_{6}\right) \delta 9.95(\mathrm{~s}, 1 \mathrm{H}, \mathrm{NH}), 9.80(\mathrm{~s}, 1 \mathrm{H}, \mathrm{NH}), 7.65(\mathrm{~s}, 1 \mathrm{H}, \mathrm{Ar}-\mathrm{H}), 7.56(\mathrm{~d}, J=8.4$ Hz, 1H, Ar-H), 6.88 (d, J=8.4 Hz, 1H, Ar-H), 4.64 (t, $J=8.4 \mathrm{~Hz}, 2 \mathrm{H}), 4.04$ (s, 2H, $\left.\mathrm{CH}_{2}\right), 3.22(\mathrm{t}, J=8.8 \mathrm{~Hz}, 2 \mathrm{H}), 1.83\left(\mathrm{~s}, 3 \mathrm{H}, \mathrm{CH}_{3}\right) ;{ }^{13} \mathrm{C}$ NMR $\left(100 \mathrm{MHz}, \mathrm{DMSO}-d_{6}\right) \delta$ $163.6,150.8,145.6,130.0,129.4,128.3,125.2,108.8,72.2,52.6,28.4,19.9$. ESI$\operatorname{HRMS}\left(\mathrm{m} / \mathrm{z}\right.$ ): Calcd. for $\mathrm{C}_{12} \mathrm{H}_{14} \mathrm{~N}_{4} \mathrm{O}_{4} \mathrm{~S}[\mathrm{M}+\mathrm{H}]^{+}$311.0809; found 331.0812.

N-(6-Methyl-3-oxo-2,3-dihydro-1,2,4-triazin-4(5H)-yl)-2,3-

dihydrobenzo[b][1,4]dioxine-6-sulfonamide (I-33). White solid, yield 86\%, Mp 213$214{ }^{\circ} \mathrm{C} .{ }^{1} \mathrm{H}$ NMR (400 MHz, DMSO-d $\left.)_{6}\right) 10.06$ (s, 1H, NH), 9.83 (s, 1H, NH), 7.27 (q, $J=2.0 \mathrm{~Hz}, 1 \mathrm{H}, \mathrm{Ar}-\mathrm{H}), 7.25(\mathrm{~d}, J=2.0 \mathrm{~Hz}, 1 \mathrm{H}, \mathrm{Ar}-\mathrm{H}), 7.00(\mathrm{~d}, J=8.4 \mathrm{~Hz}, 1 \mathrm{H}, \mathrm{Ar}-$ $\mathrm{H}), 4.32\left(\mathrm{~d}, J=5.2 \mathrm{~Hz}, 2 \mathrm{H}, \mathrm{OCH}_{2}\right), 4.29\left(\mathrm{~d}, J=5.2 \mathrm{~Hz}, 2 \mathrm{H}, \mathrm{OCH}_{2}\right), 4.03\left(\mathrm{~s}, 2 \mathrm{H}, \mathrm{CH}_{2}\right)$, $1.83\left(\mathrm{~s}, 3 \mathrm{H}, \mathrm{CH}_{3}\right) ;{ }^{13} \mathrm{C}$ NMR (100 MHz, DMSO- $\left.d_{6}\right) \delta 150.7,147.5,145.6,143.0,131.0$, 121.5, 117.3, 116.9, 64.5, 64.0, 52.6, 19.9. ESI-HRMS(m/z): Calcd. for $\mathrm{C}_{12} \mathrm{H}_{14} \mathrm{~N}_{4} \mathrm{O}_{5} \mathrm{~S}$ $[\mathrm{M}+\mathrm{H}]^{+}$327.0758; found 327.0760.

\section{Synthesis of $\quad N$-(6-methyl-3-oxo-2,3-dihydro-1,2,4-triazin-4(5H)-yl)- $N$ -}

(methylsulfonyl)methanesulfonamide (II-1). To a solution of amino triazinone (1)

(0.26 g, $2 \mathrm{mmol})$ in pyridine $(30 \mathrm{~mL})$ was added methylsufonyl chloride (2-1) $(0.50 \mathrm{~g}$, $4.4 \mathrm{mmol}$ ), and the resulting mixture was stirred for $10 \mathrm{~h}$ at room temperature. The solution was concentrated under reduced pressure and the residue was diluted by water (50 mL). The mixture was extracted with $\mathrm{CH}_{2} \mathrm{Cl}_{2}$, and the extract was washed with brine, and dried over anhydrous $\mathrm{Na}_{2} \mathrm{SO}_{4}$. The crude product was purified by flash chromatography on silica gel using dichloromethane and methanol $(\mathrm{v} / \mathrm{v}=150: 1)$ as eluent to give compound II-1 as a white solid (0.48 g, yield 85\%). Mp 220-222 ${ }^{\circ} \mathrm{C} .{ }^{1} \mathrm{H}$ 
NMR (400 MHz, DMSO- $\left.d_{6}\right) \delta 10.34(\mathrm{~s}, 1 \mathrm{H}, \mathrm{NH}), 4.39\left(\mathrm{~s}, 2 \mathrm{H}, \mathrm{CH}_{2}\right), 3.56\left(\mathrm{~s}, 6 \mathrm{H}, \mathrm{CH}_{3}\right)$, $1.89\left(\mathrm{~s}, 3 \mathrm{H}, \mathrm{CH}_{3}\right) ;{ }^{13} \mathrm{C}$ NMR (100 MHz, DMSO- $\left.d_{6}\right) \delta 149.9,146.0,54.3,44.8,19.7$. ESI-HRMS(m/z): Calcd. for $\mathrm{C}_{6} \mathrm{H}_{13} \mathrm{~N}_{4} \mathrm{O}_{5} \mathrm{~S}_{2}[\mathrm{M}+\mathrm{H}]^{+}$285.0322; found 285.0323.

Compounds II-2-II-8 were synthesized using the similar procedure as compound II-1.

$N$-(Ethylsulfonyl)-N-(6-methyl-3-oxo-2,3-dihydro-1,2,4-triazin-4(5H)-

yl)ethanesulfonamide (II-2). White solid, yield 74\%, Mp 183-184 ${ }^{\circ} \mathrm{C} .{ }^{1} \mathrm{H}$ NMR $(300$ $\left.\mathrm{MHz}, \mathrm{DMSO}-d_{6}\right) \delta 10.33(\mathrm{~s}, 1 \mathrm{H}, \mathrm{NH}), 4.31\left(\mathrm{~s}, 2 \mathrm{H}, \mathrm{CH}_{2}\right), 3.64(\mathrm{q}, J=7.5 \mathrm{~Hz}, 4 \mathrm{H}$, $\left.\mathbf{C H}_{2} \mathrm{CH}_{3}\right), 1.89\left(\mathrm{~s}, 3 \mathrm{H}, \mathrm{CH}_{3}\right), 1.33\left(\mathrm{t}, J=9.6 \mathrm{~Hz}, 6 \mathrm{H}, \mathrm{CH}_{2} \mathbf{C H}_{3}\right) ;{ }^{13} \mathrm{C} \mathrm{NMR}(100 \mathrm{MHz}$, DMSO- $\left.d_{6}\right) \delta 150.1,146.1,54.0,51.3,19.8$, 7.6. ESI-HRMS(m/z): Calcd. for $\mathrm{C}_{8} \mathrm{H}_{17} \mathrm{~N}_{4} \mathrm{O}_{5} \mathrm{~S}_{2}[\mathrm{M}+\mathrm{H}]^{+}$313.0635; found 313.0633.

N-(6-Methyl-3-oxo-2,3-dihydro-1,2,4-triazin-4(5H)-yl)-N-

(propylsulfonyl)propane-1-sulfonamide (II-3). White solid, yield 84\%, Mp 177-178 ${ }^{\circ} \mathrm{C}$. ${ }^{1} \mathrm{H}$ NMR $\left(300 \mathrm{MHz}, \mathrm{DMSO}-d_{6}\right) \delta 10.32(\mathrm{~s}, 1 \mathrm{H}, \mathrm{NH}), 4.33\left(\mathrm{~s}, 2 \mathrm{H}, \mathrm{CH}_{2}\right), 3.54-3.69(\mathrm{~m}$, $\left.4 \mathrm{H}, \mathbf{C H}_{2} \mathrm{CH}_{2} \mathrm{CH}_{3}\right), 1.89\left(\mathrm{~s}, 3 \mathrm{H}, \mathrm{CH}_{3}\right), 1.77-1.86\left(\mathrm{~m}, 4 \mathrm{H}, \mathrm{CH}_{2} \mathbf{C H}_{2} \mathrm{CH}_{3}\right), 1.00(\mathrm{t}, J=7.5$ $\left.\mathrm{Hz}, 6 \mathrm{H}, \mathrm{CH}_{2} \mathrm{CH}_{2} \mathbf{C H}_{3}\right) ;{ }^{13} \mathrm{C} \mathrm{NMR}\left(100 \mathrm{MHz}, \mathrm{DMSO}-d_{6}\right) \delta 150.1,146.0,58.2,54.1$, 19.8, 16.5, 12.5. ESI-HRMS(m/z): Calcd. for $\mathrm{C}_{10} \mathrm{H}_{21} \mathrm{~N}_{4} \mathrm{O}_{5} \mathrm{~S}_{2}[\mathrm{M}+\mathrm{H}]^{+}$341.0948; found 341.0946.

N-(Butylsulfonyl)-N-(6-methyl-3-oxo-2,3-dihydro-1,2,4-triazin-4(5H)-yl)butane-

1-sulfonamide (II-4). White solid, yield 85\%, Mp 157-158 ${ }^{\circ} \mathrm{C} .{ }^{1} \mathrm{H}$ NMR (300 MHz, DMSO- $\left.d_{6}\right) \delta 10.33(\mathrm{~s}, 1 \mathrm{H}, \mathrm{NH}), 4.33\left(\mathrm{~s}, 2 \mathrm{H}, \mathrm{CH}_{2}\right), 3.56-3.72\left(\mathrm{~m}, 4 \mathrm{H}, \mathbf{C H}_{2} \mathrm{CH}_{2} \mathrm{CH}_{2} \mathrm{H}_{3}\right)$, $1.90\left(\mathrm{~s}, \quad 3 \mathrm{H}, \quad \mathrm{CH}_{3}\right), \quad 1.71-1.83\left(\mathrm{~m}, \quad 4 \mathrm{H}, \quad \mathrm{CH}_{2} \mathbf{C H}_{2} \mathrm{CH}_{2} \mathrm{H}_{3}\right), 1.38-1.46 \quad(\mathrm{~m}, \quad 4 \mathrm{H}$, 
$\mathrm{CH}_{2} \mathrm{CH}_{2} \mathbf{C H}_{2} \mathrm{H}_{3}$ ), 0.9 (t, J = 7.2 Hz, 6H, $\left.\mathrm{CH}_{2} \mathrm{CH}_{2} \mathrm{CH}_{2} \mathbf{C H}_{3}\right) ;{ }^{13} \mathrm{C}$ NMR (100 MHz, DMSO- $\left.d_{6}\right) \delta 150.1,146.0,56.4,54.1,24.5,20.7,19.8,13.3$. ESI-HRMS(m/z): Calcd. for $\mathrm{C}_{12} \mathrm{H}_{25} \mathrm{~N}_{4} \mathrm{O}_{5} \mathrm{~S}_{2}[\mathrm{M}+\mathrm{H}]^{+}$369.1261; found 369.1263.

$N$-(6-Methyl-3-oxo-2,3-dihydro-1,2,4-triazin-4(5H)-yl)- $N$ -

(phenylsulfonyl)benzenesulfonamide (II-5). White solid, yield 83\%, Mp 227-228 ${ }^{\circ} \mathrm{C}$. ${ }^{1} \mathrm{H}$ NMR (400 MHz, DMSO- $\left.d_{6}\right) \delta 10.25$ (s, $\left.1 \mathrm{H}, \mathrm{NH}\right), 7.90(\mathrm{~d}, J=7.5 \mathrm{~Hz}, 4 \mathrm{H}, \mathrm{Ar}-\mathrm{H})$, $7.83(\mathrm{t}, J=7.5 \mathrm{~Hz}, 2 \mathrm{H}, \mathrm{Ar}-\mathrm{H}), 7.68(\mathrm{t}, J=7.8 \mathrm{~Hz}, 4 \mathrm{H}, \mathrm{Ar}-\mathrm{H}), 4.22\left(\mathrm{~s}, 2 \mathrm{H}, \mathrm{CH}_{2}\right), 1.87$ $\left(\mathrm{s}, 3 \mathrm{H}, \mathrm{CH}_{3}\right) ;{ }^{13} \mathrm{C}$ NMR $\left(100 \mathrm{MHz}, \mathrm{DMSO}-d_{6}\right) \delta 149.5,146.3,137.6,135.1,129.5$, 128.6, 53.1, 19.9. ESI-HRMS(m/z): Calcd. for $\mathrm{C}_{16} \mathrm{H}_{17} \mathrm{~N}_{4} \mathrm{O}_{5} \mathrm{~S}_{2}[\mathrm{M}+\mathrm{H}]^{+}$409.0635; found 409.0639 .

4-Methyl-N-(6-methyl-3-oxo-2,3-dihydro-1,2,4-triazin-4(5H)-yl)-N-

tosylbenzenesulfonamide (II-6). White solid, yield 81\%, Mp 219-220 ${ }^{\circ} \mathrm{C} .{ }^{1} \mathrm{H}$ NMR $\left(300 \mathrm{MHz}, \mathrm{DMSO}-d_{6}\right) \delta 10.22(\mathrm{~s}, 1 \mathrm{H}, \mathrm{NH}), 7.77(\mathrm{~d}, J=8.1 \mathrm{~Hz}, 4 \mathrm{H}, \mathrm{Ar}-\mathrm{H}), 7.47(\mathrm{~d}, J$ $=8.1 \mathrm{~Hz}, 4 \mathrm{H}, \mathrm{Ar}-\mathrm{H}), 4.20\left(\mathrm{~s}, 2 \mathrm{H}, \mathrm{CH}_{2}\right), 2.44\left(\mathrm{~s}, 6 \mathrm{H}, \mathrm{CH}_{3}\right), 1.87\left(\mathrm{~s}, 3 \mathrm{H}, \mathrm{CH}_{3}\right) ;{ }^{13} \mathrm{C} \mathrm{NMR}$ $\left(100 \mathrm{MHz}, \mathrm{DMSO}-d_{6}\right) \delta 149.5,146.3,145.9,134.8,129.8,128.7,53.0,21.2,19.9$. ESI$\operatorname{HRMS}(\mathrm{m} / \mathrm{z})$ : Calcd. for $\mathrm{C}_{18} \mathrm{H}_{21} \mathrm{~N}_{4} \mathrm{O}_{5} \mathrm{~S}_{2}[\mathrm{M}+\mathrm{H}]^{+} 437.0948$; found 437.0947.

N-(6-Methyl-3-oxo-2,3-dihydro-1,2,4-triazin-4(5H)-yl)-4-acetamino-N-(4acetaminophenylsulfonyl)benzenesulfonamide(II-7). White solid, yield 78\%, Mp 189$190{ }^{\circ} \mathrm{C} .{ }^{1} \mathrm{H}$ NMR $\left(300 \mathrm{MHz}, \mathrm{DMSO}-d_{6}\right) \delta 10.51(\mathrm{~s}, 2 \mathrm{H}, \mathrm{NH}), 10.18(\mathrm{~s}, 1 \mathrm{H}, \mathrm{NH}), 7.80$ (s, 8H, Ar-H), 4.20(s, 2H, $\left.\mathrm{CH}_{2}\right), 2.12\left(\mathrm{~s}, 6 \mathrm{H}, \mathrm{CH}_{3}\right), 1.87\left(\mathrm{~s}, 3 \mathrm{H}, \mathrm{CH}_{3}\right) ;{ }^{13} \mathrm{C}$ NMR $(100$ MHz, DMSO- $\left.d_{6}\right) \delta 169.4,149.5,146.2,144.9,130.6,130.1,118.3,53.1,24.3,19.9$. ESI-HRMS(m/z): Calcd. for $\mathrm{C}_{20} \mathrm{H}_{23} \mathrm{~N}_{6} \mathrm{O}_{7} \mathrm{~S}_{2}[\mathrm{M}+\mathrm{H}]^{+}$540.1330; found 540.1332. 
N-(6-Methyl-3-oxo-2,3-dihydro-1,2,4-triazin-4(5H)-yl)-4-nitro-N-(4-

nitrophenylsulfonyl)benzenesulfonamide (II-8). White solid, yield 79\%, Mp 239$240{ }^{\circ} \mathrm{C} .{ }^{1} \mathrm{H}$ NMR $\left(300 \mathrm{MHz}, \mathrm{DMSO}-d_{6}\right) \delta 10.37(\mathrm{~s}, 1 \mathrm{H}, \mathrm{NH}), 8.49(\mathrm{~d}, J=9.0 \mathrm{~Hz}, 4 \mathrm{H}$, $\mathrm{Ar}-\mathrm{H}), 8.20(\mathrm{~d}, J=9.0 \mathrm{~Hz}, 4 \mathrm{H}, \mathrm{Ar}-\mathrm{H}), 4.41\left(\mathrm{~s}, 2 \mathrm{H}, \mathrm{CH}_{2}\right), 1.93\left(\mathrm{~s}, 3 \mathrm{H}, \mathrm{CH}_{3}\right) ;{ }^{13} \mathrm{C} \mathrm{NMR}$ $\left(100 \mathrm{MHz}, \mathrm{DMSO}-d_{6}\right) \delta 151.1,149.1,146.4,142.3,130.6,124.8,53.6,19.9$. ESI$\operatorname{HRMS}(\mathrm{m} / \mathrm{z})$ : Calcd. for $\mathrm{C}_{16} \mathrm{H}_{15} \mathrm{~N}_{6} \mathrm{O}_{9} \mathrm{~S}_{2}[\mathrm{M}+\mathrm{H}]^{+} 499.0336$; found 499.0329. 
NMR Data of I-1-I-33 and II-1-II-8

I-1 ${ }^{1} \mathrm{H}$ NMR (400 MHz, DMSO- $d_{6}$ )

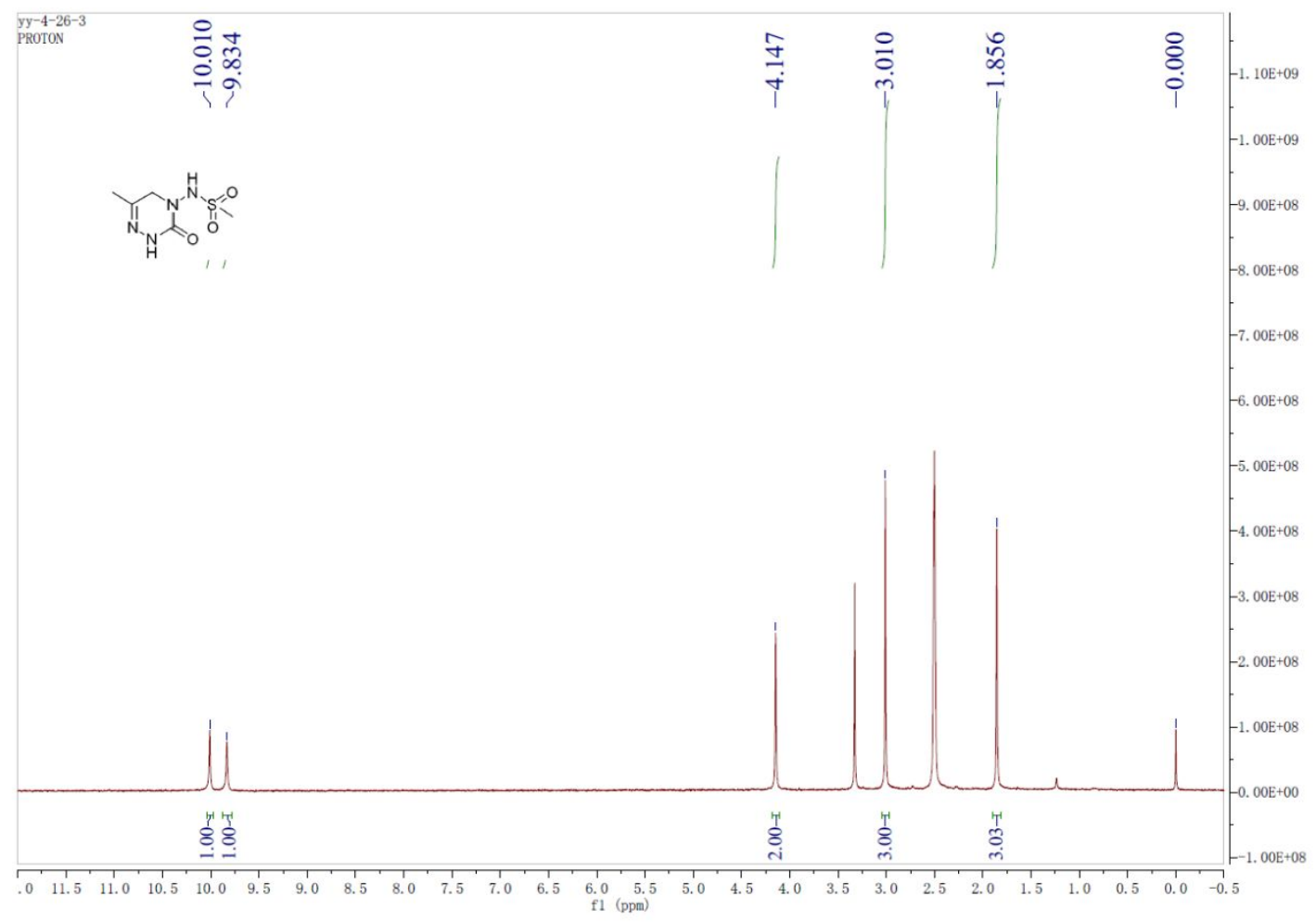

I-1 ${ }^{13} \mathrm{C}$ NMR $\left(100 \mathrm{MHz}, \mathrm{DMSO}-d_{6}\right)$

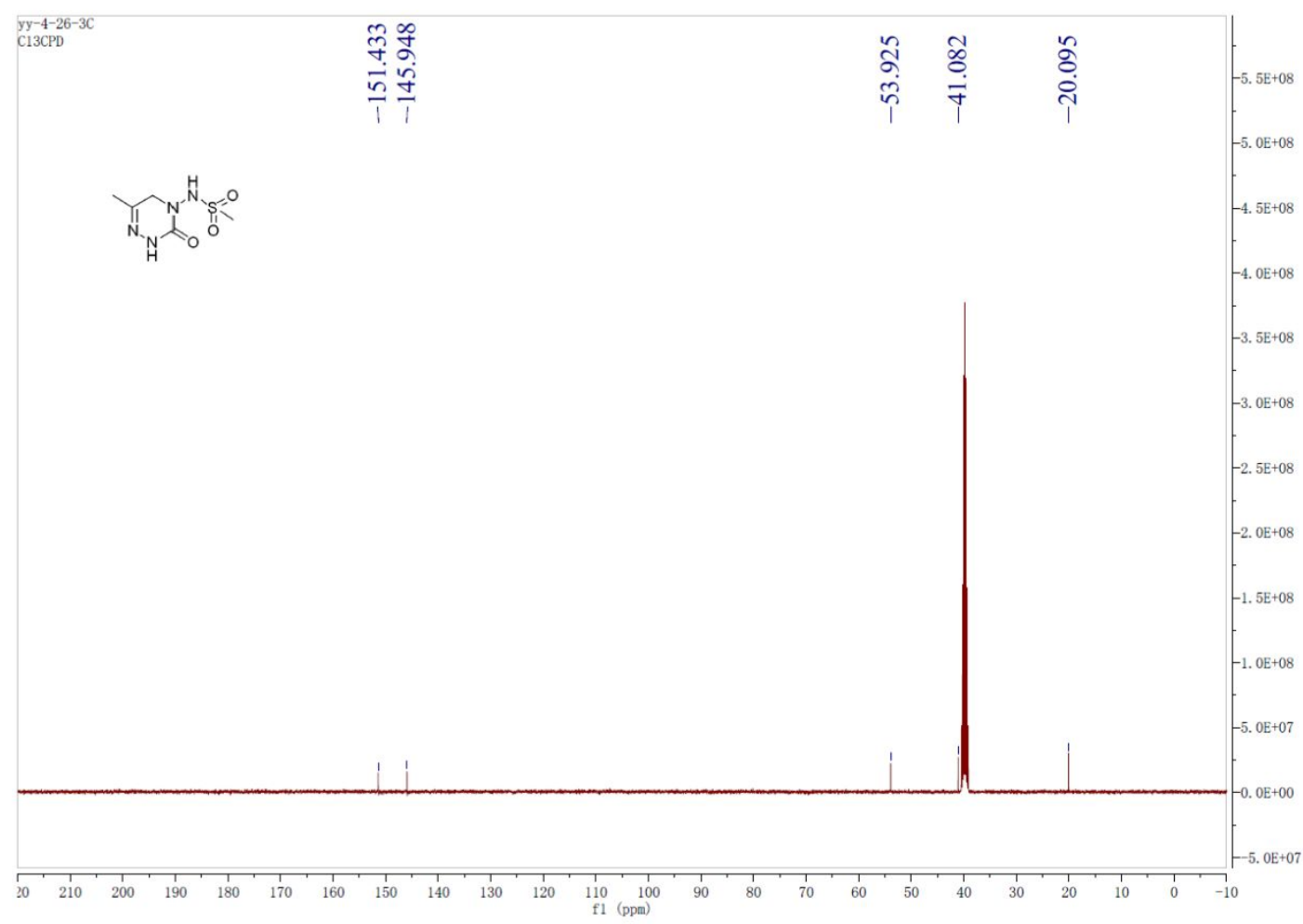


I-2 ${ }^{1} \mathrm{H}$ NMR (300 MHz, DMSO- $\left.d_{6}\right)$

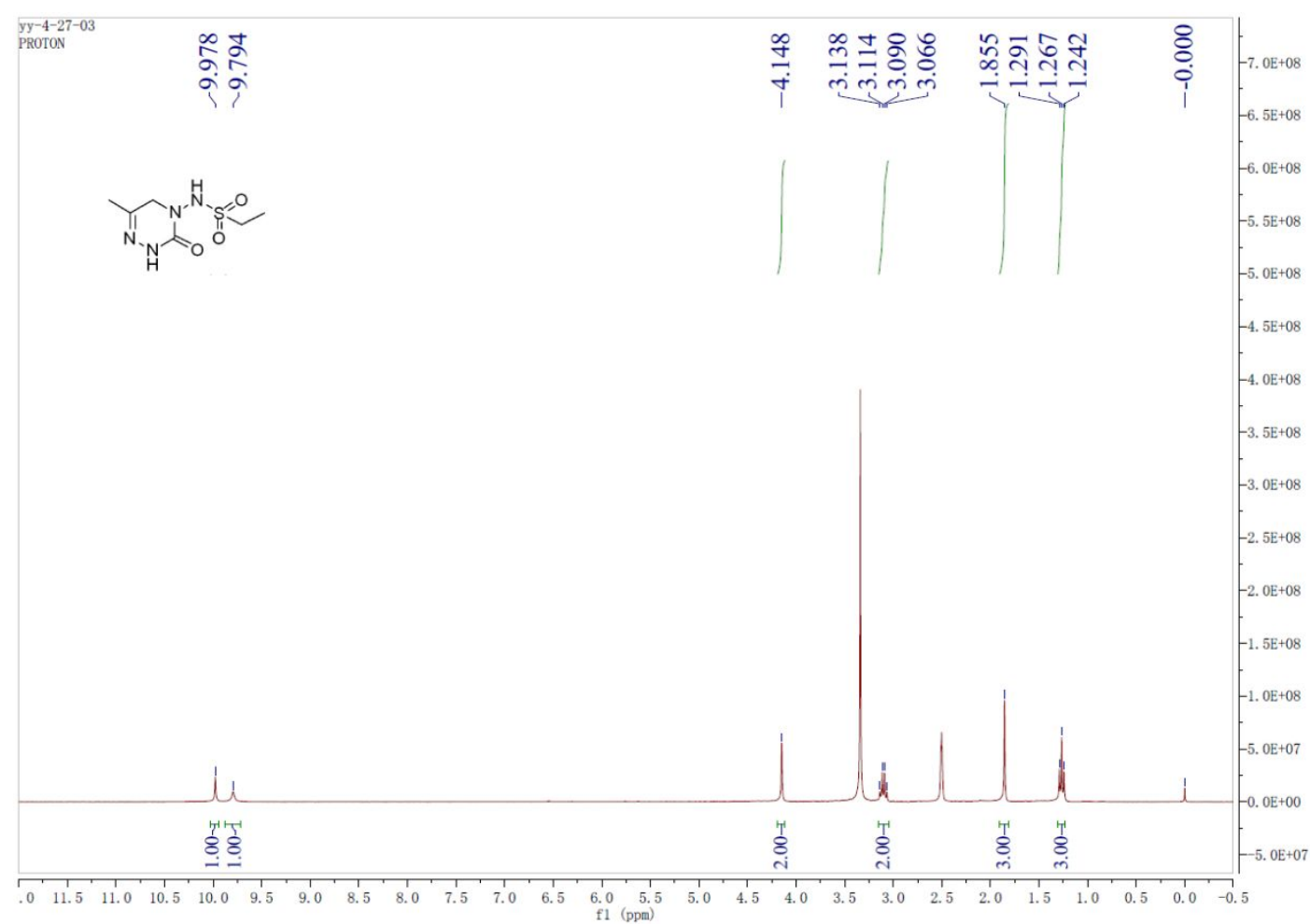

I-2 ${ }^{13} \mathrm{C}$ NMR (100 MHz, DMSO- $\left.d_{6}\right)$

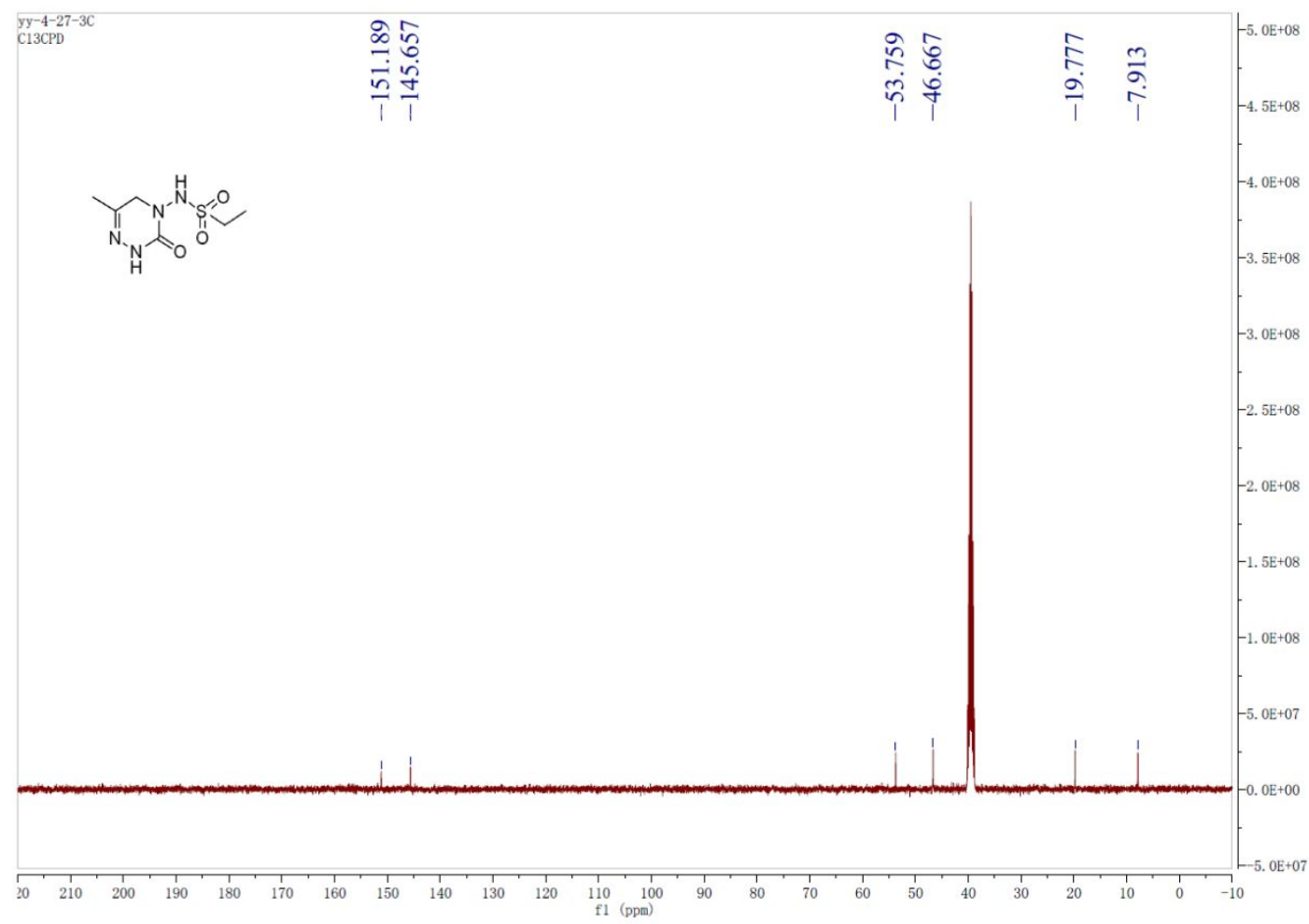


I-3 ${ }^{1} \mathrm{H}$ NMR (300 MHz, DMSO- $\left.d_{6}\right)$

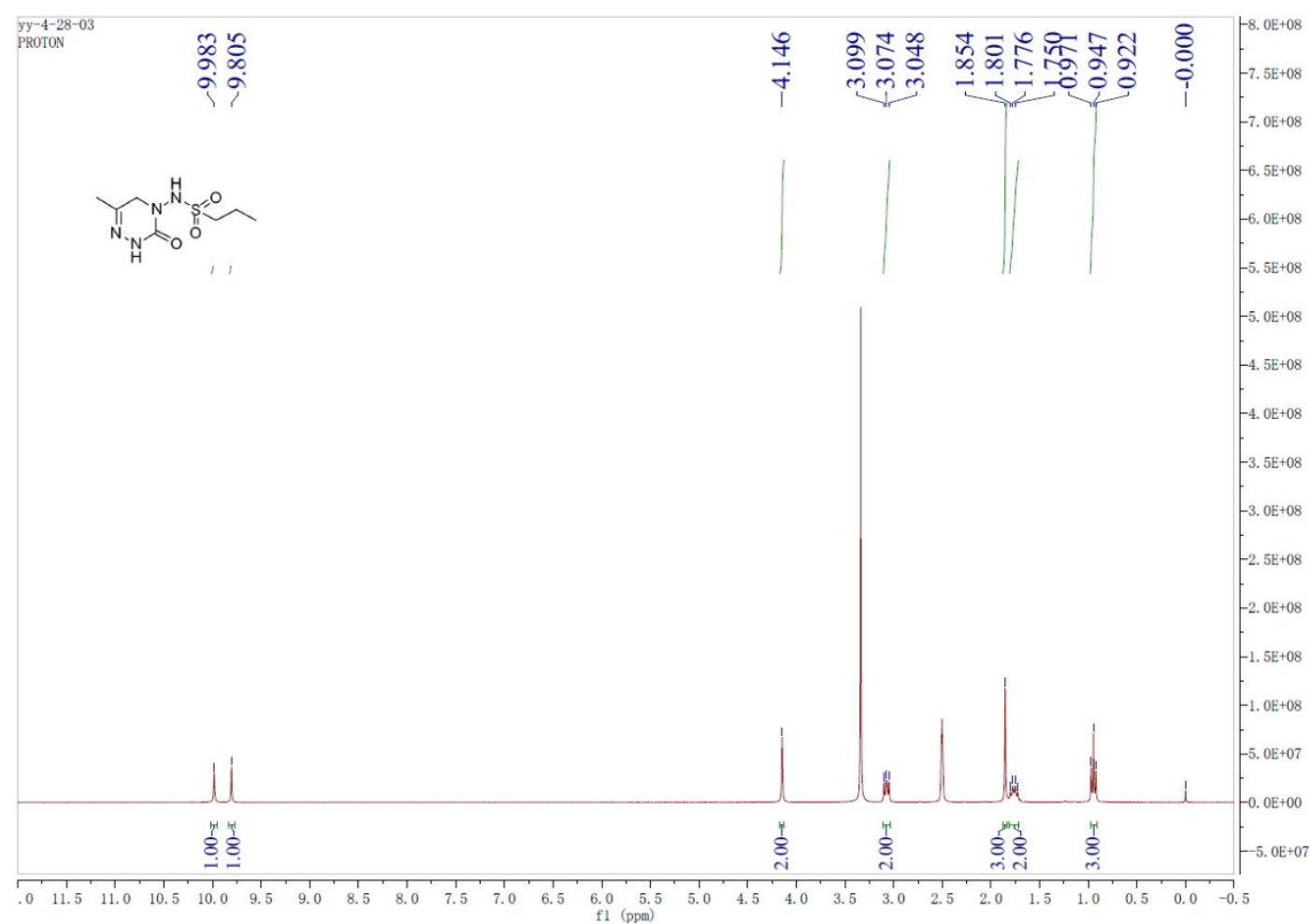

I-3 ${ }^{13} \mathrm{C}$ NMR (100 MHz, DMSO- $\left.d_{6}\right)$

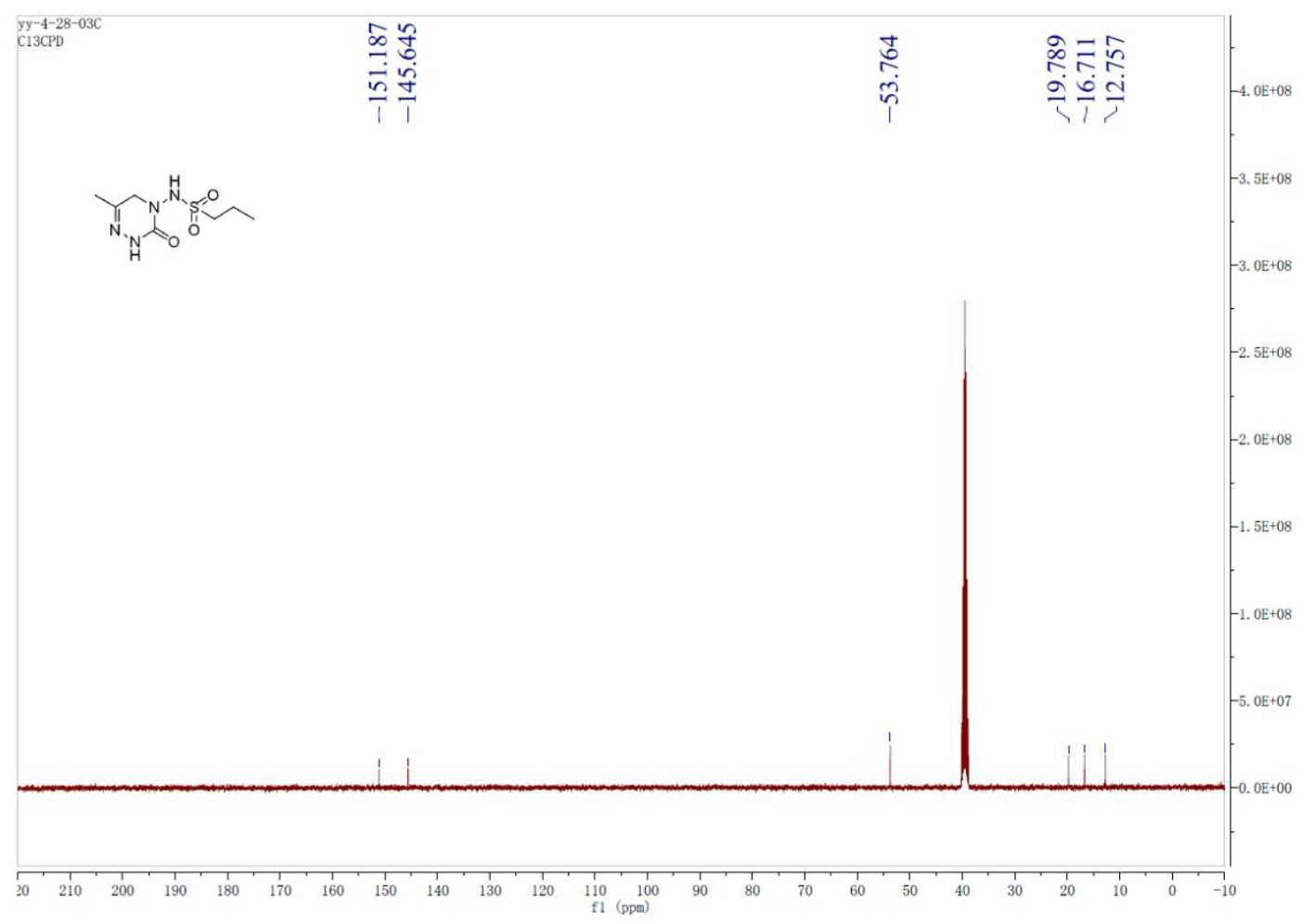


I-4 ${ }^{1} \mathrm{H}$ NMR (300 MHz, DMSO- $\left.d_{6}\right)$

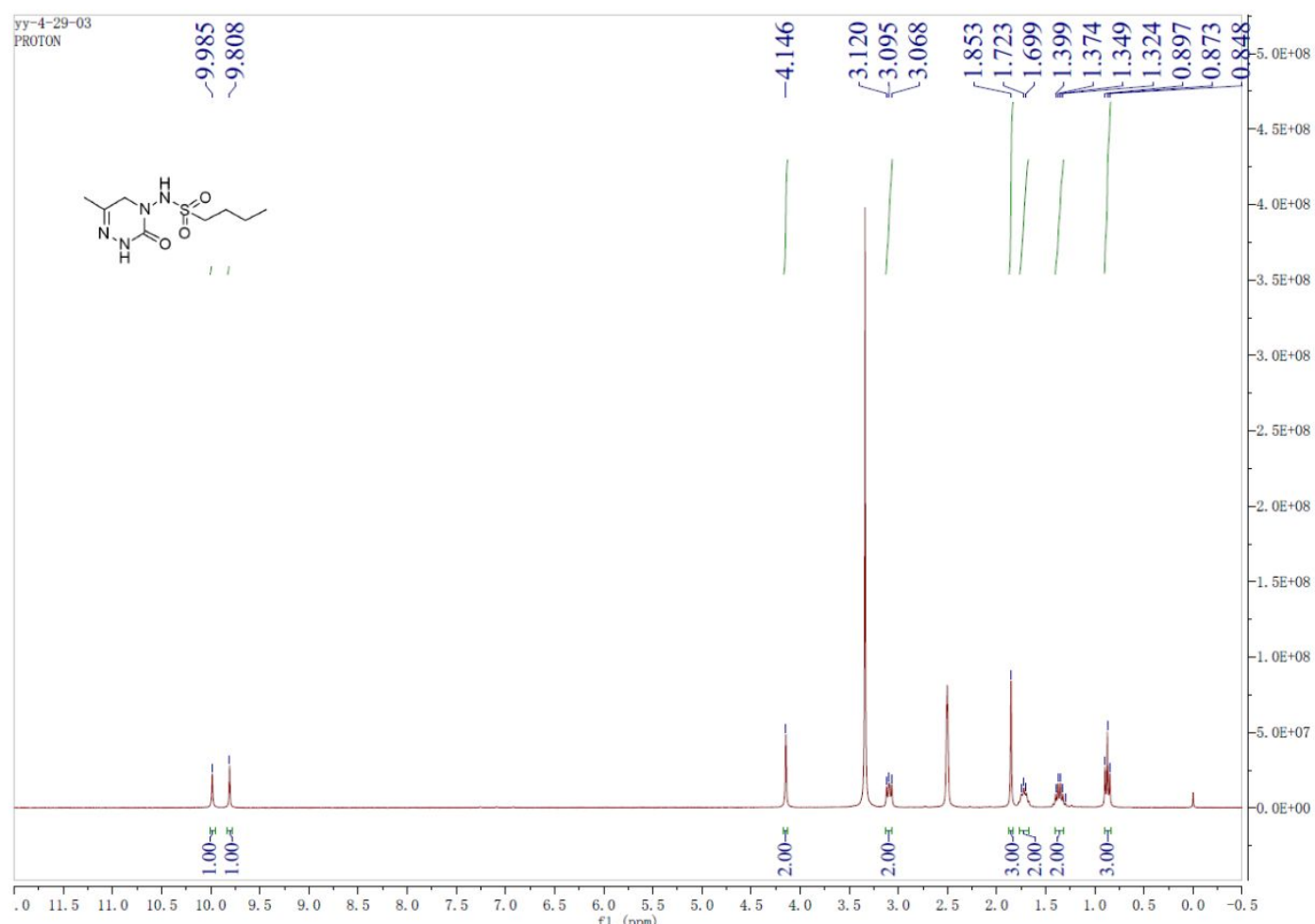

I-4 ${ }^{13} \mathrm{C}$ NMR (100 MHz, DMSO- $\left.d_{6}\right)$

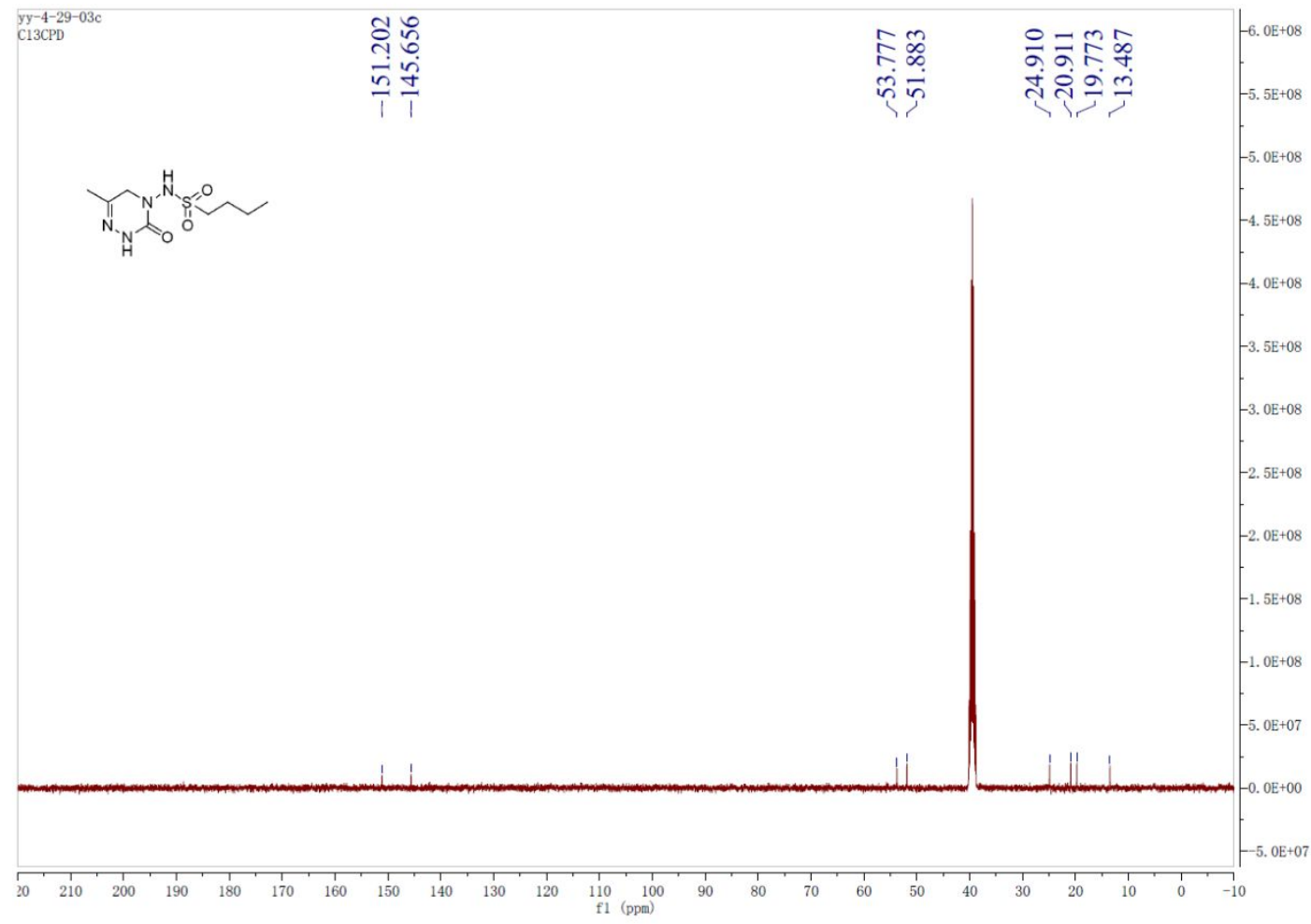


I-5 ${ }^{1} \mathrm{H}$ NMR $\left(400 \mathrm{MHz}\right.$, DMSO- $\left.d_{6}\right)$

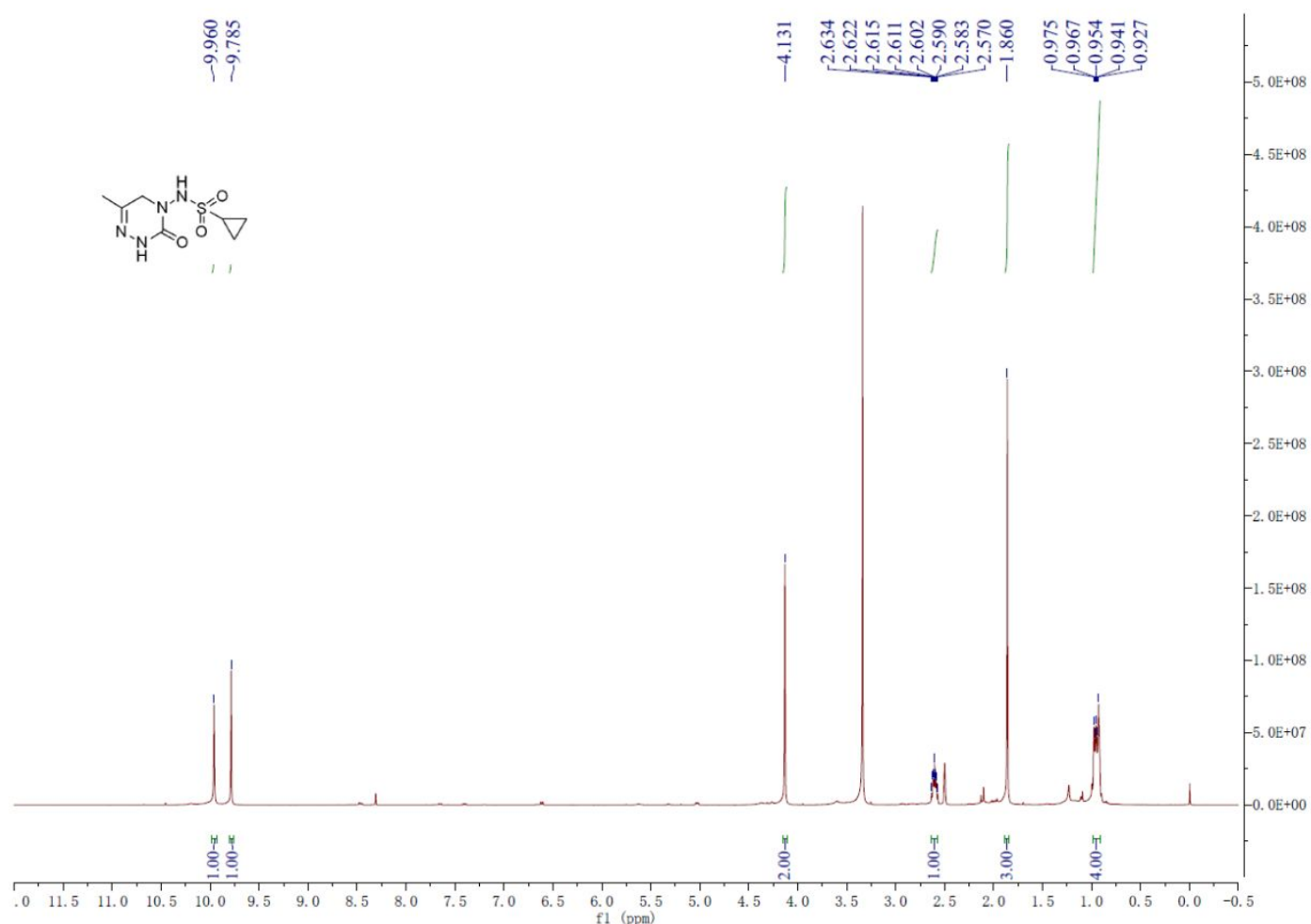

I-5 ${ }^{13} \mathrm{C}$ NMR (100 MHz, DMSO- $\left.d_{6}\right)$

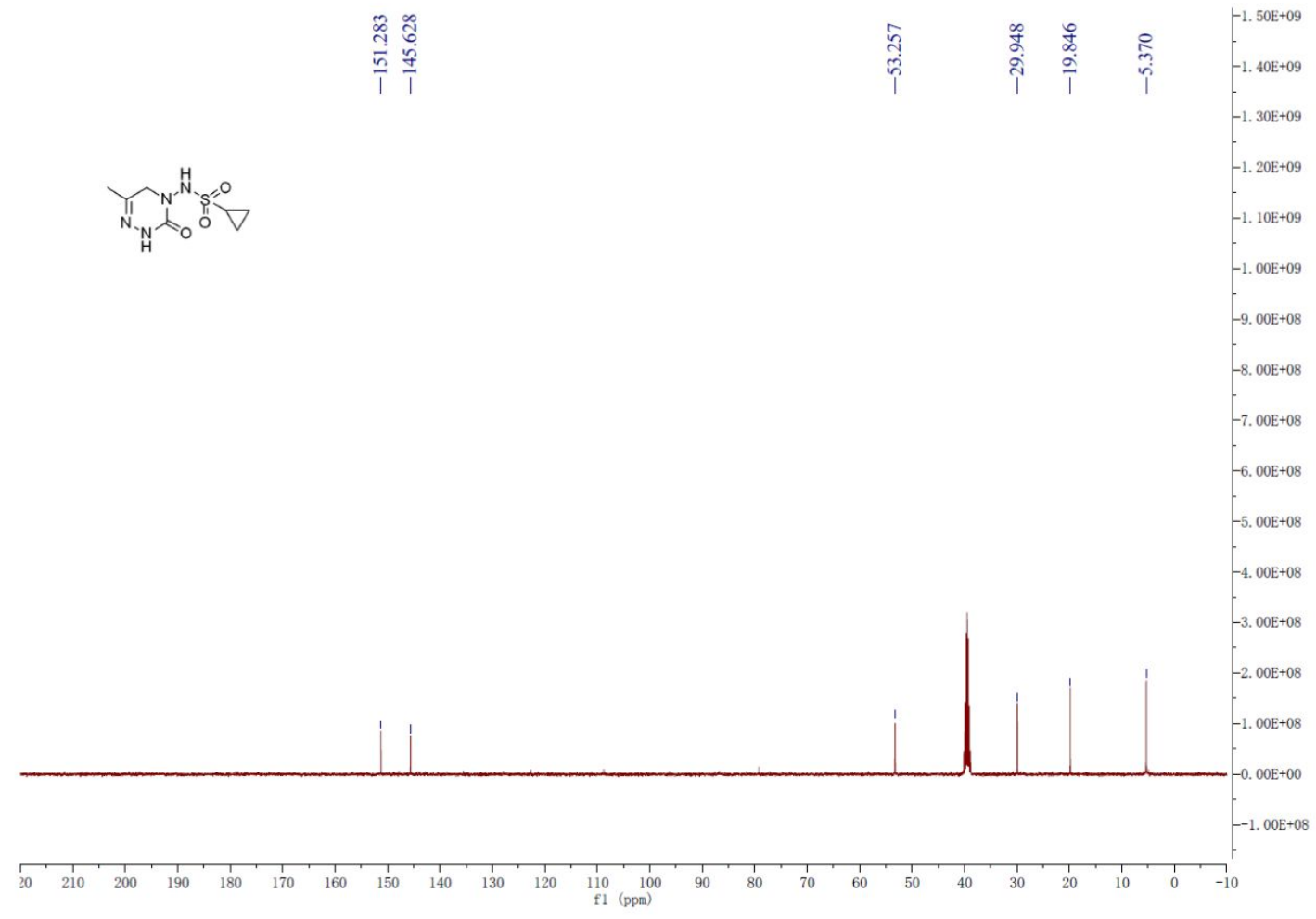


I-6 ${ }^{1} \mathrm{H}$ NMR (400 MHz, DMSO- $\left.d_{6}\right)$

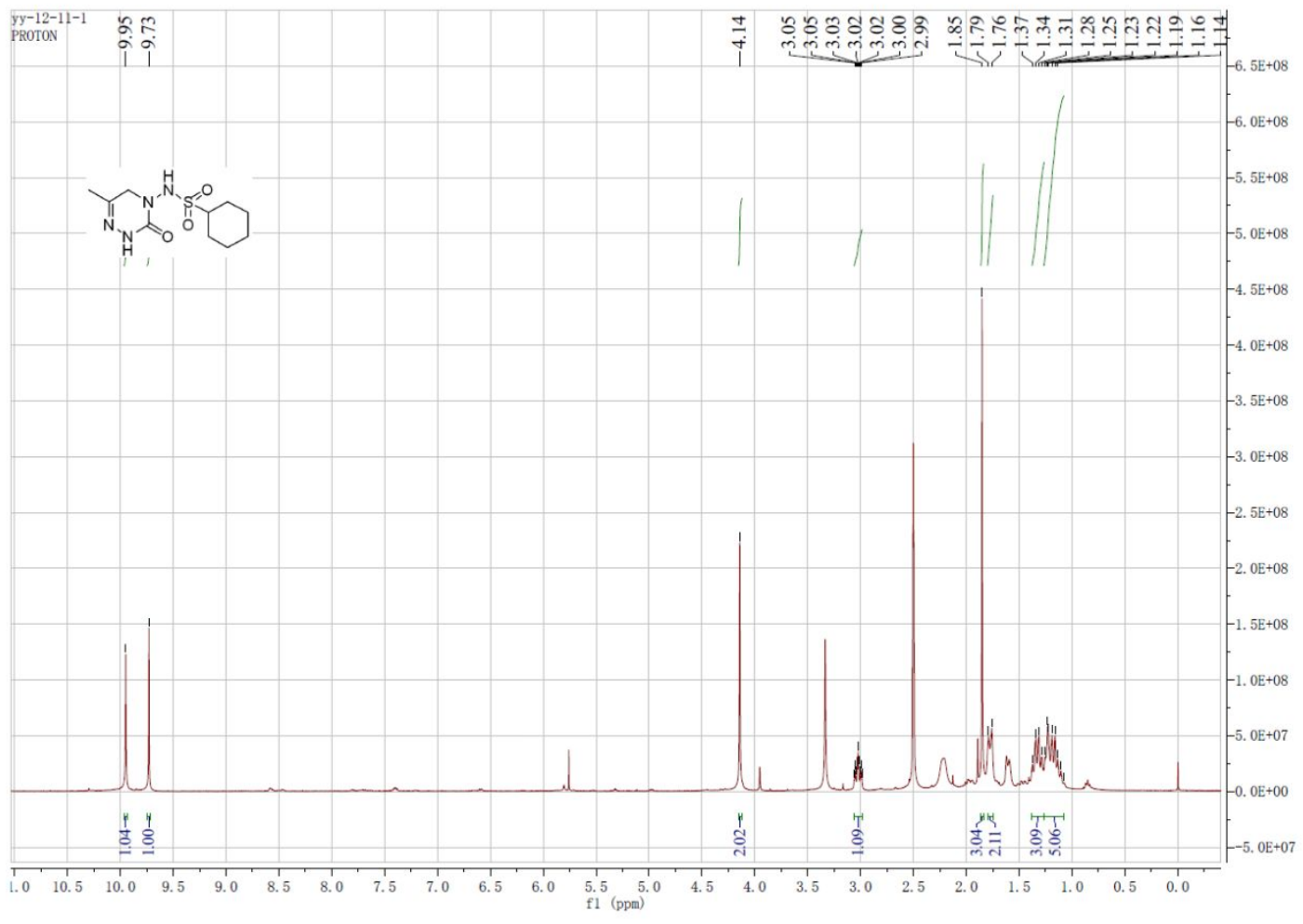

I-6 ${ }^{13} \mathrm{C}$ NMR (100 MHz, DMSO- $\left.d_{6}\right)$

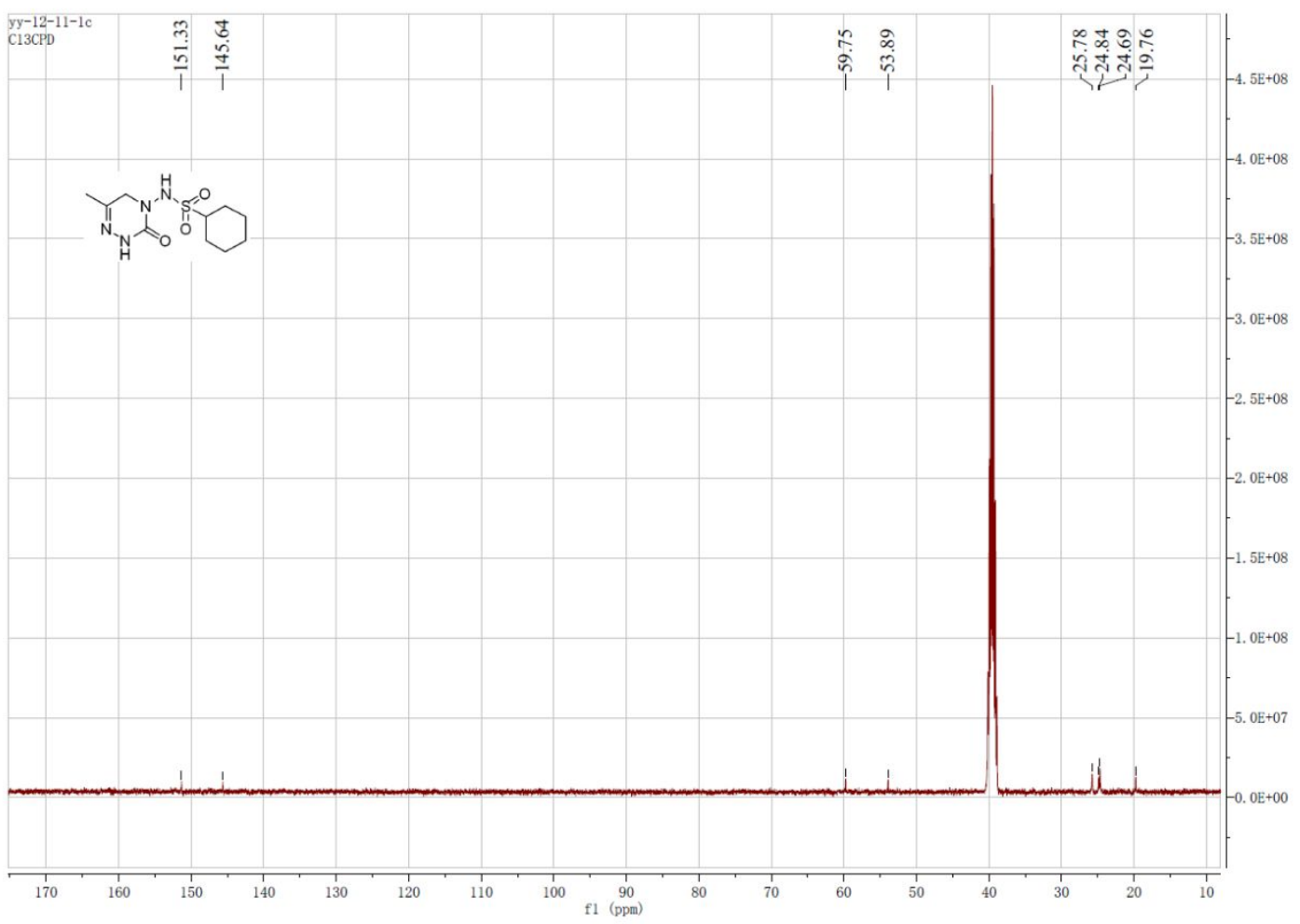

S22 
I-7 ${ }^{1} \mathrm{H}$ NMR (300 MHz, DMSO- $\left.d_{6}\right)$

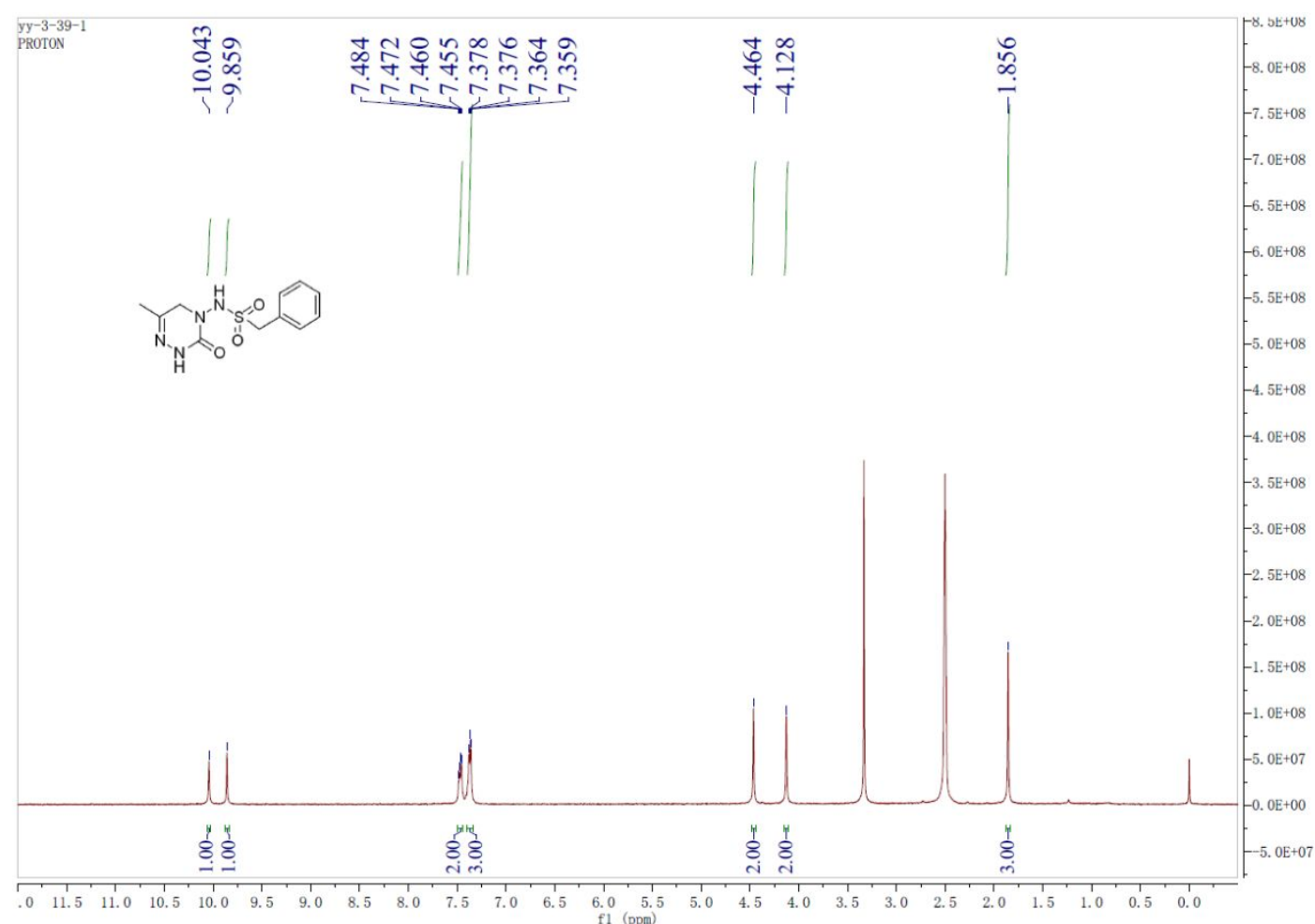

I-7 ${ }^{13} \mathrm{C}$ NMR (100 MHz, DMSO- $\left.d_{6}\right)$

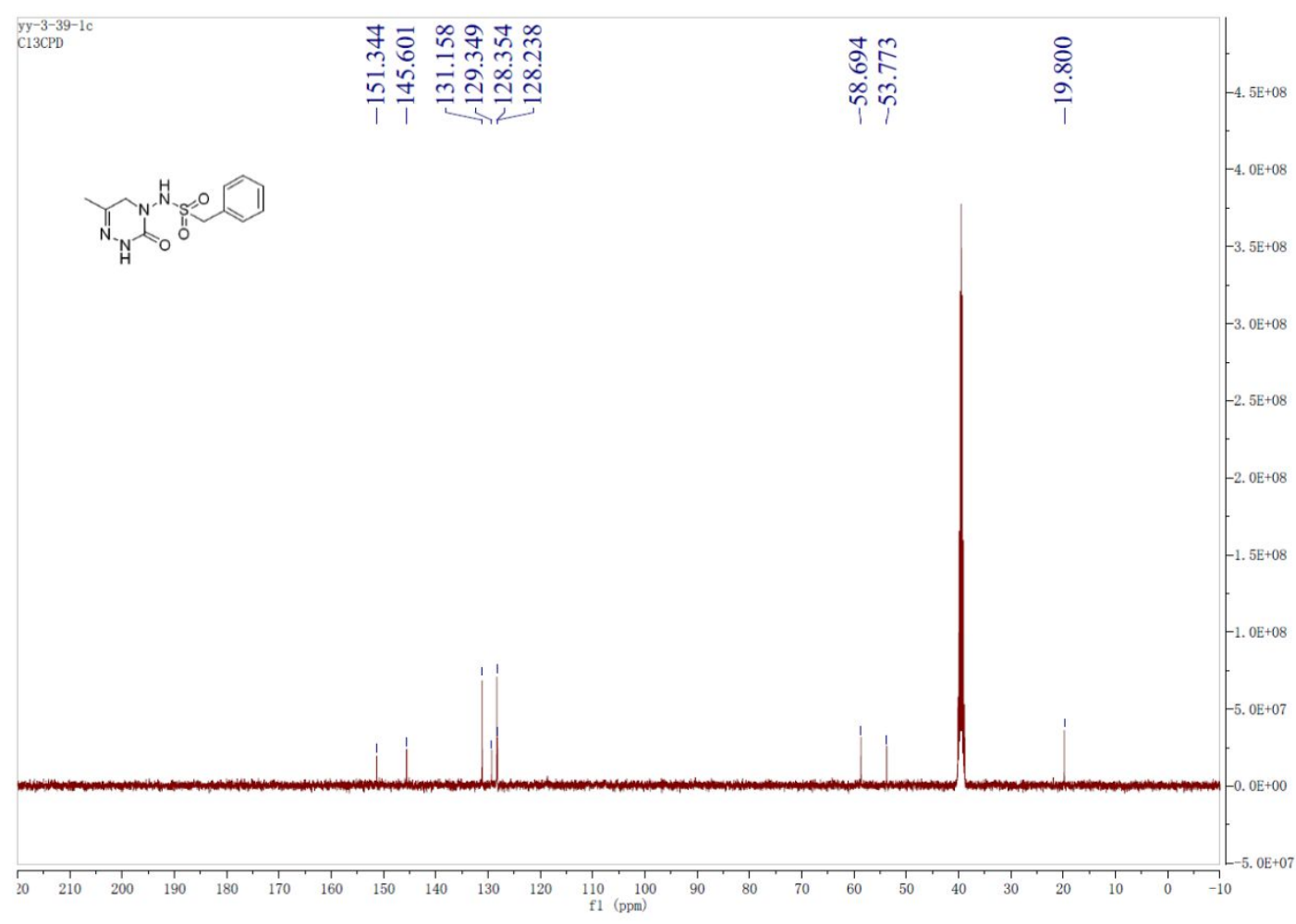


I-8 ${ }^{1} \mathrm{H}$ NMR (400 MHz, DMSO- $d_{6}$ )

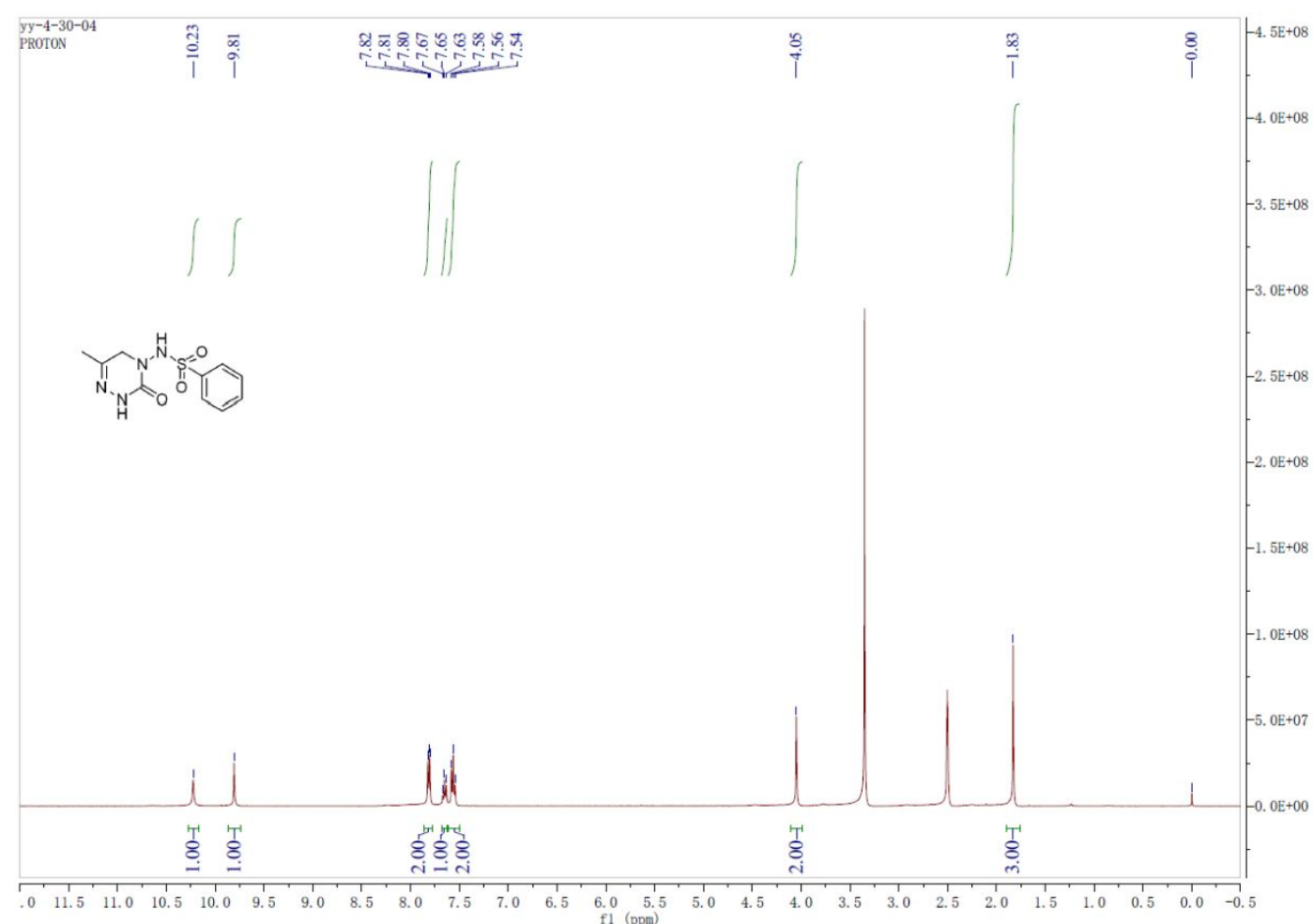

I-8 ${ }^{13} \mathrm{C}$ NMR (100 MHz, DMSO- $\left.d_{6}\right)$

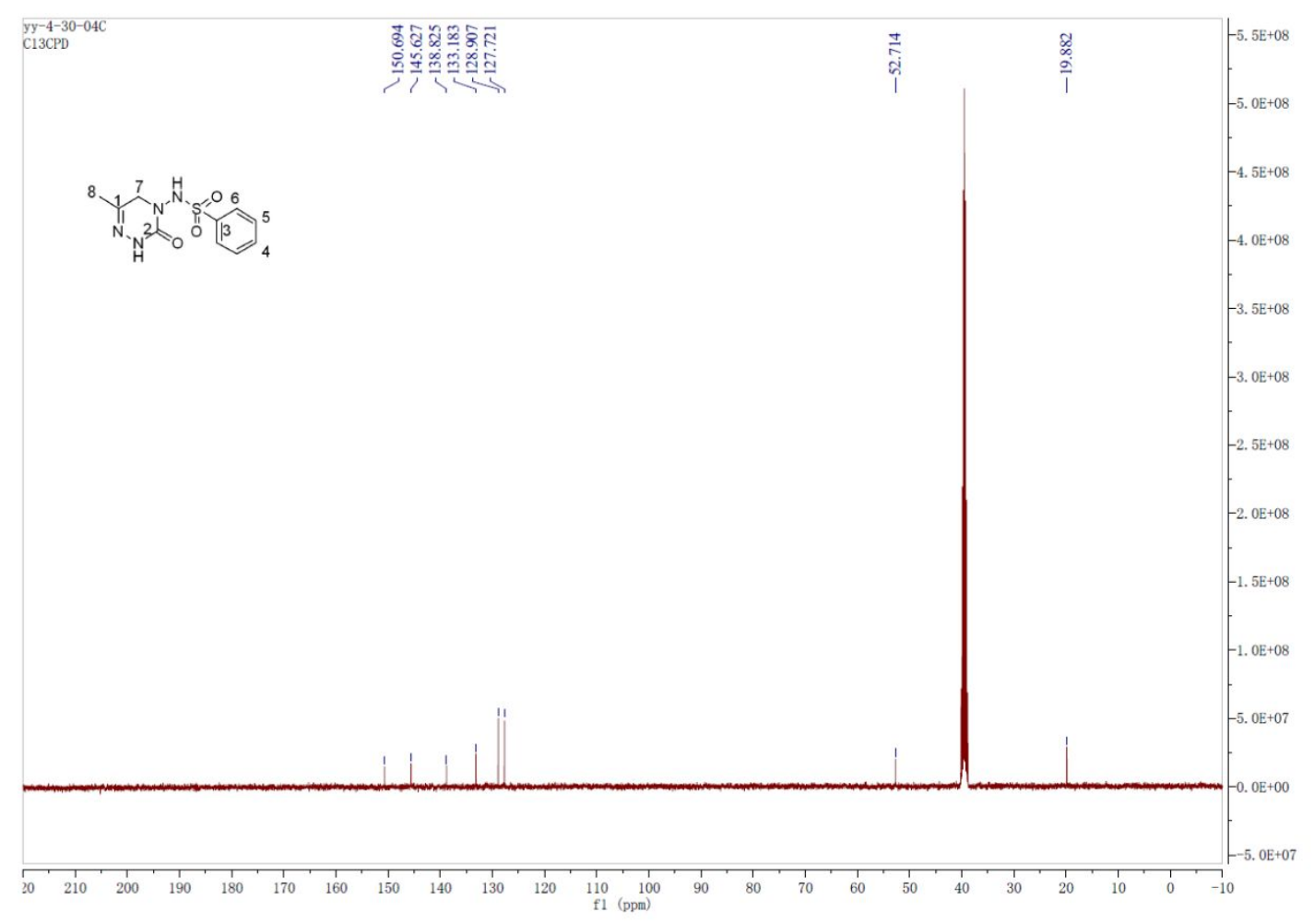


I-9 ${ }^{1} \mathrm{H}$ NMR (400 MHz, DMSO- $\left.d_{6}\right)$

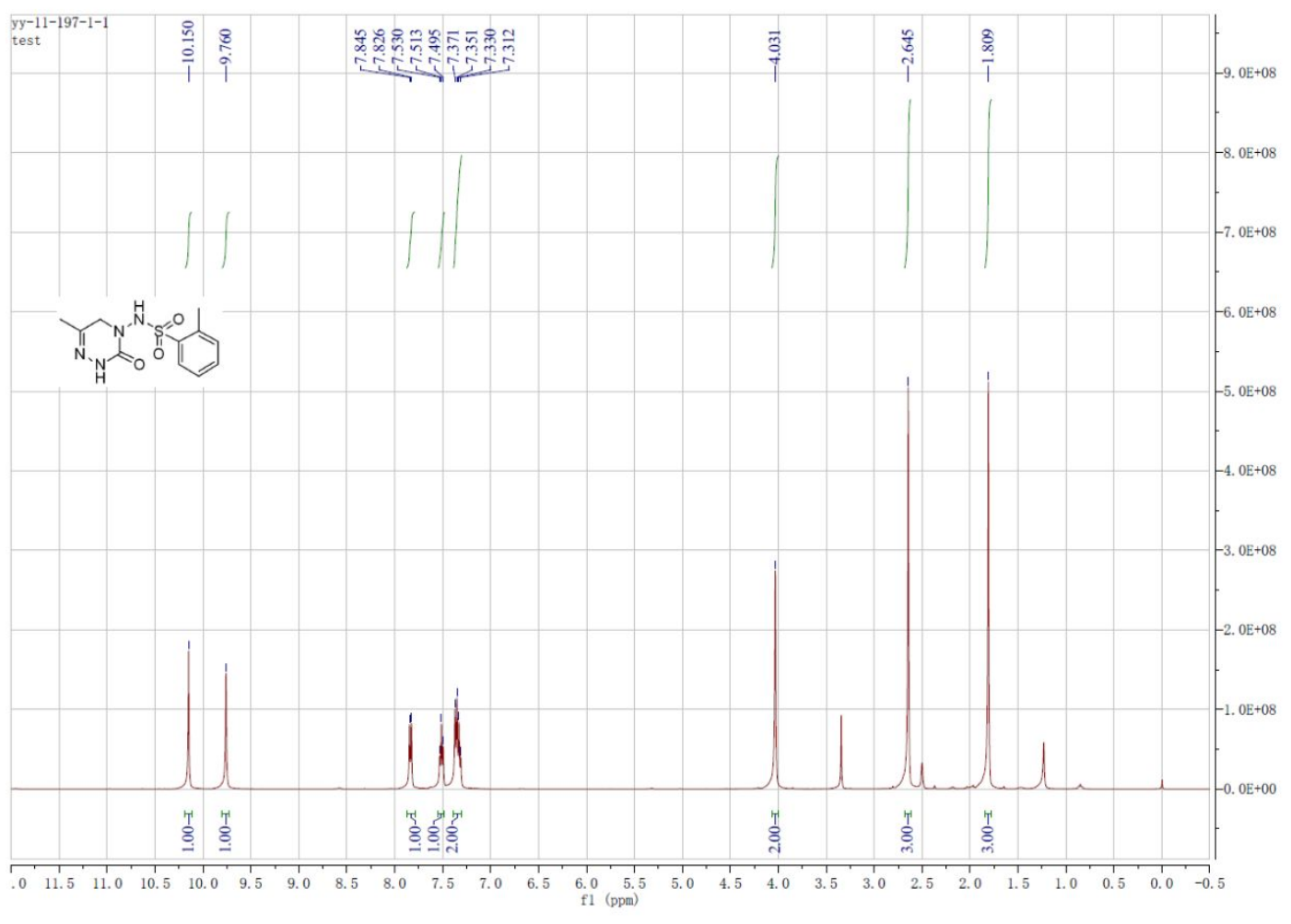

I-9 ${ }^{13} \mathrm{C}$ NMR (100 MHz, DMSO- $\left.d_{6}\right)$

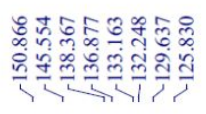

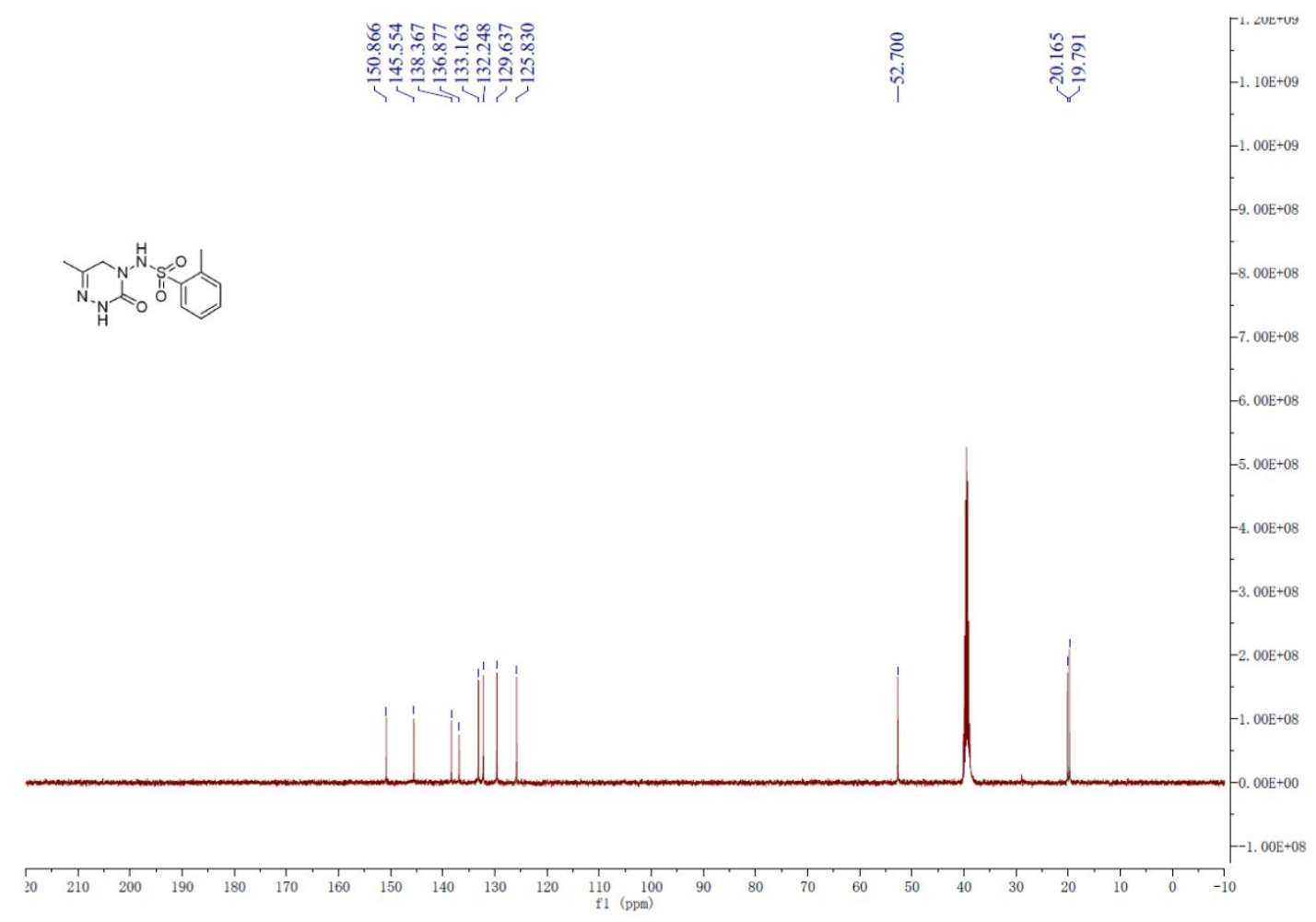

S25 
I-10 ${ }^{1} \mathrm{H}$ NMR (400 MHz, DMSO- $\left.d_{6}\right)$

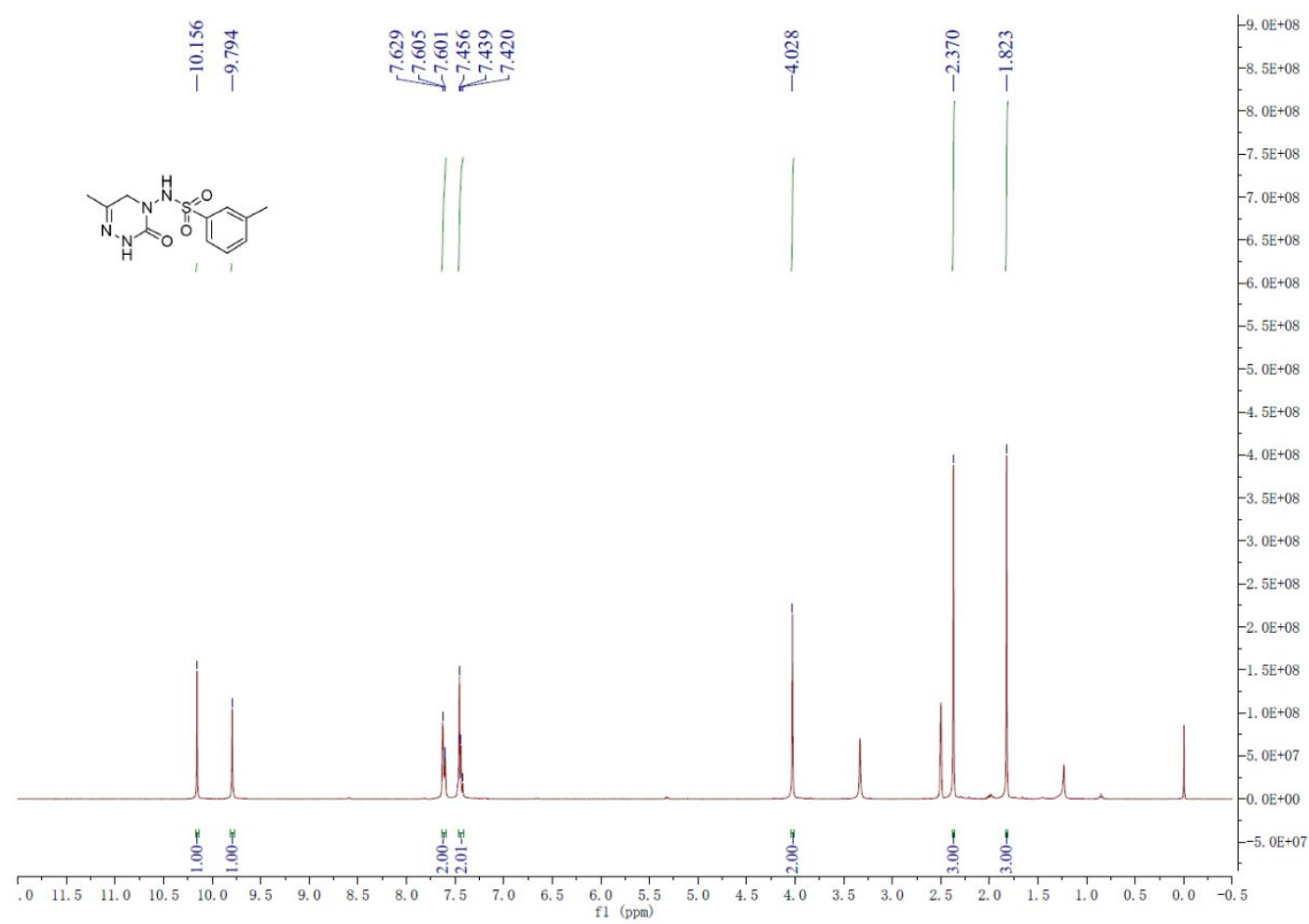

\section{I-10 ${ }^{13} \mathrm{C}$ NMR (100 MHz, DMSO- $\left.d_{6}\right)$}

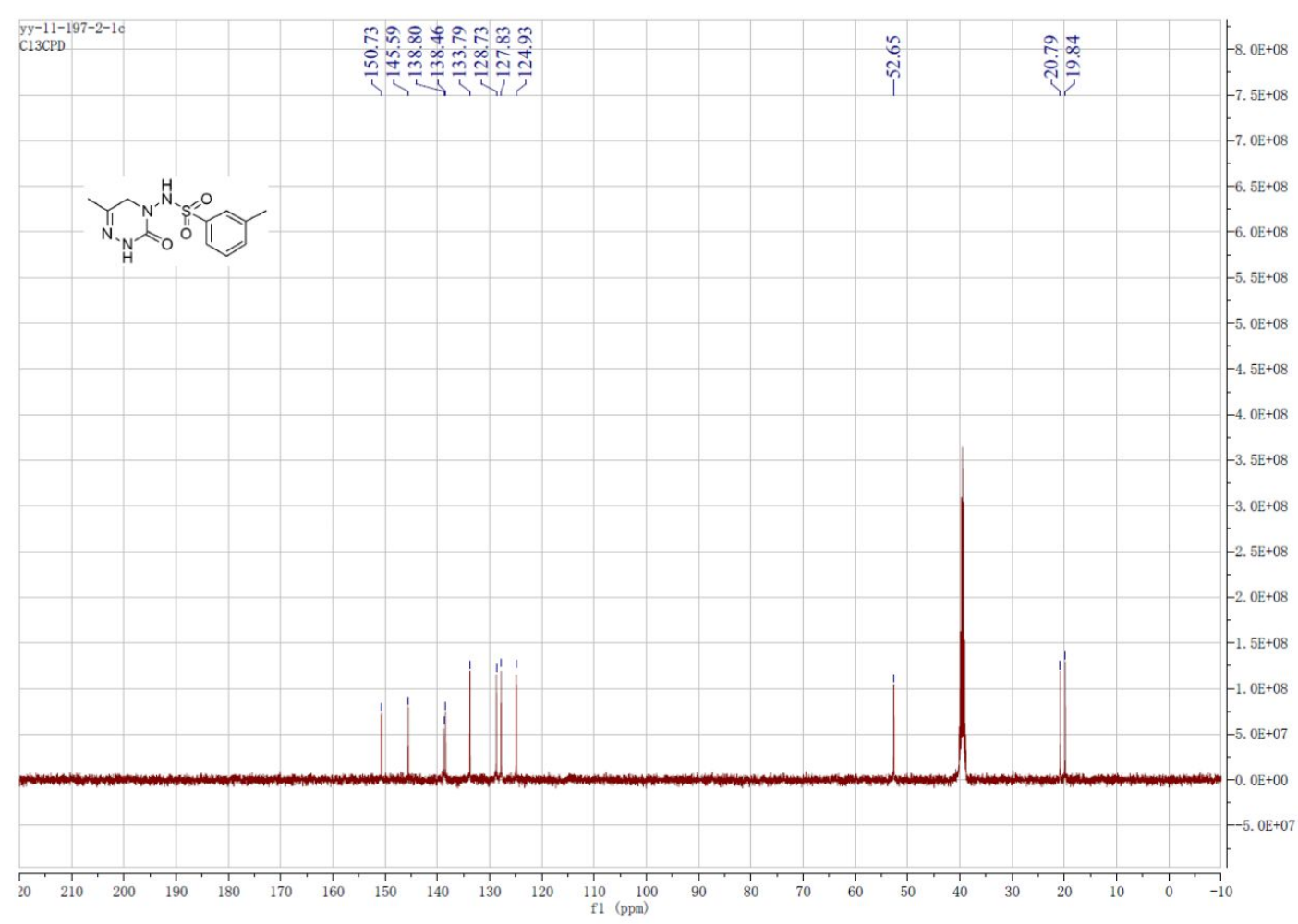


I-10 ${ }^{1} \mathrm{H}$ NMR (300 MHz, DMSO- $\left.d_{6}\right)$

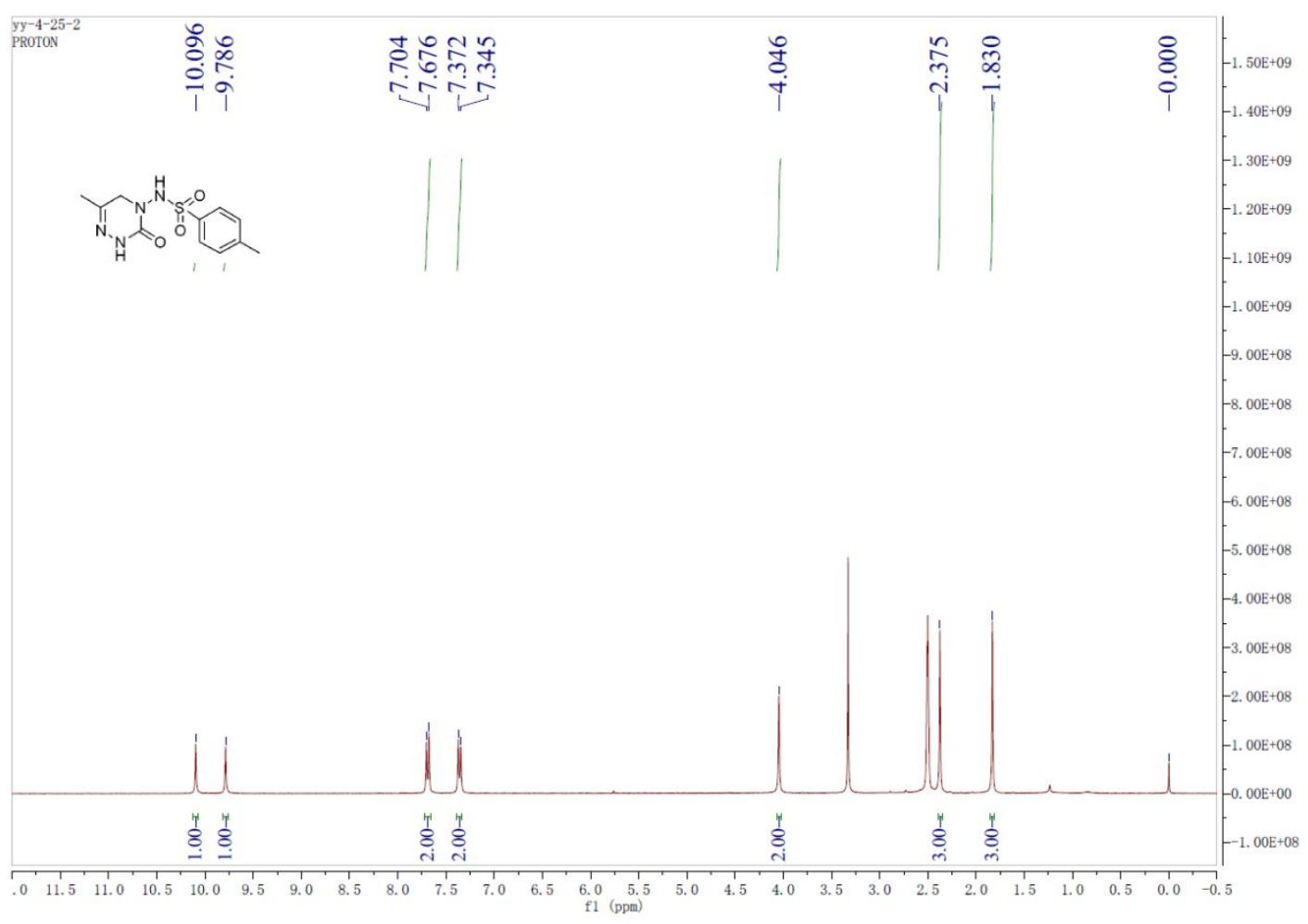

I-11 ${ }^{13} \mathrm{C}$ NMR $\left(100 \mathrm{MHz}, \mathrm{DMSO}-d_{6}\right)$

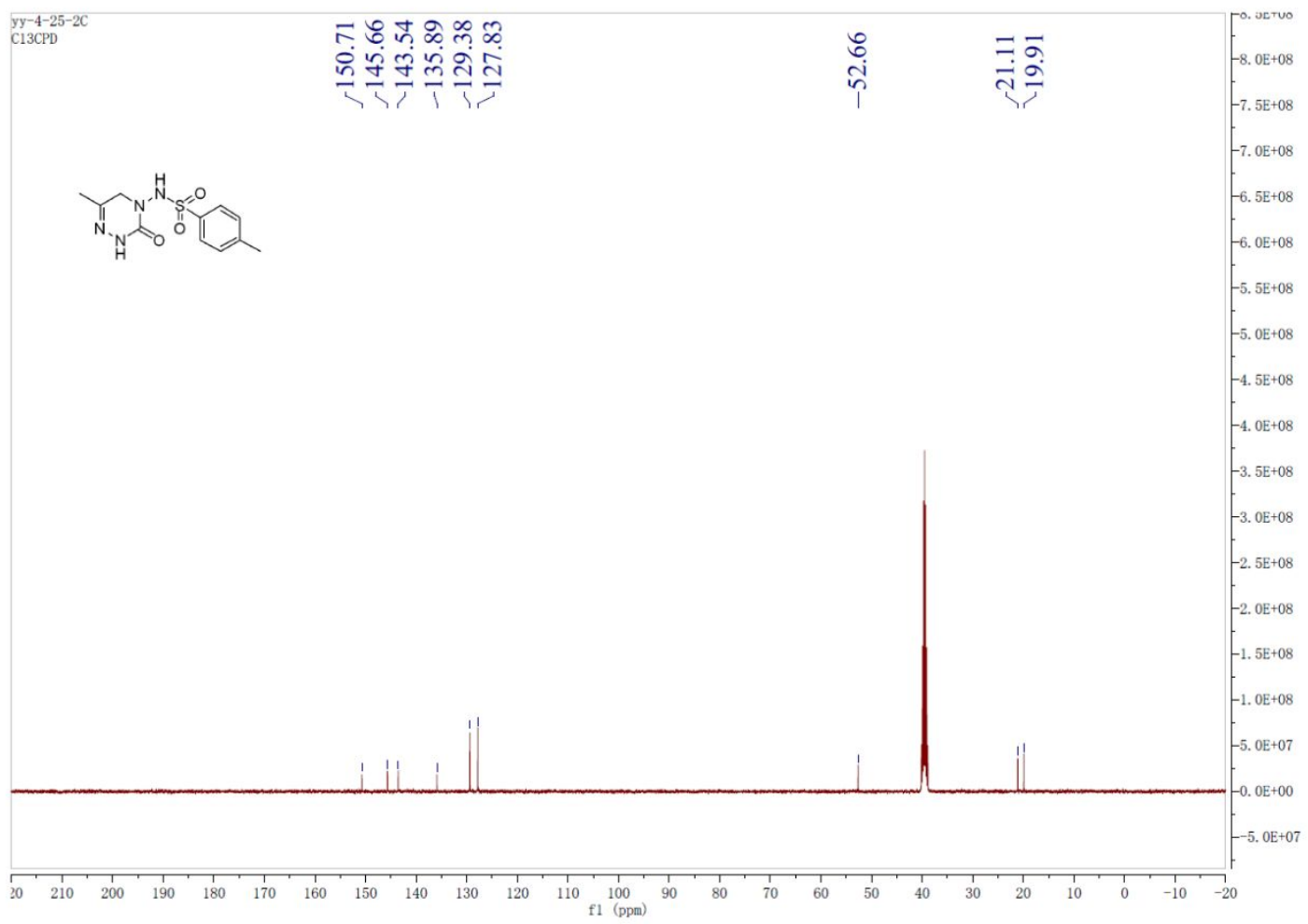

S27 
I-12 ${ }^{1} \mathrm{H}$ NMR (400 MHz, DMSO- $\left.d_{6}\right)$

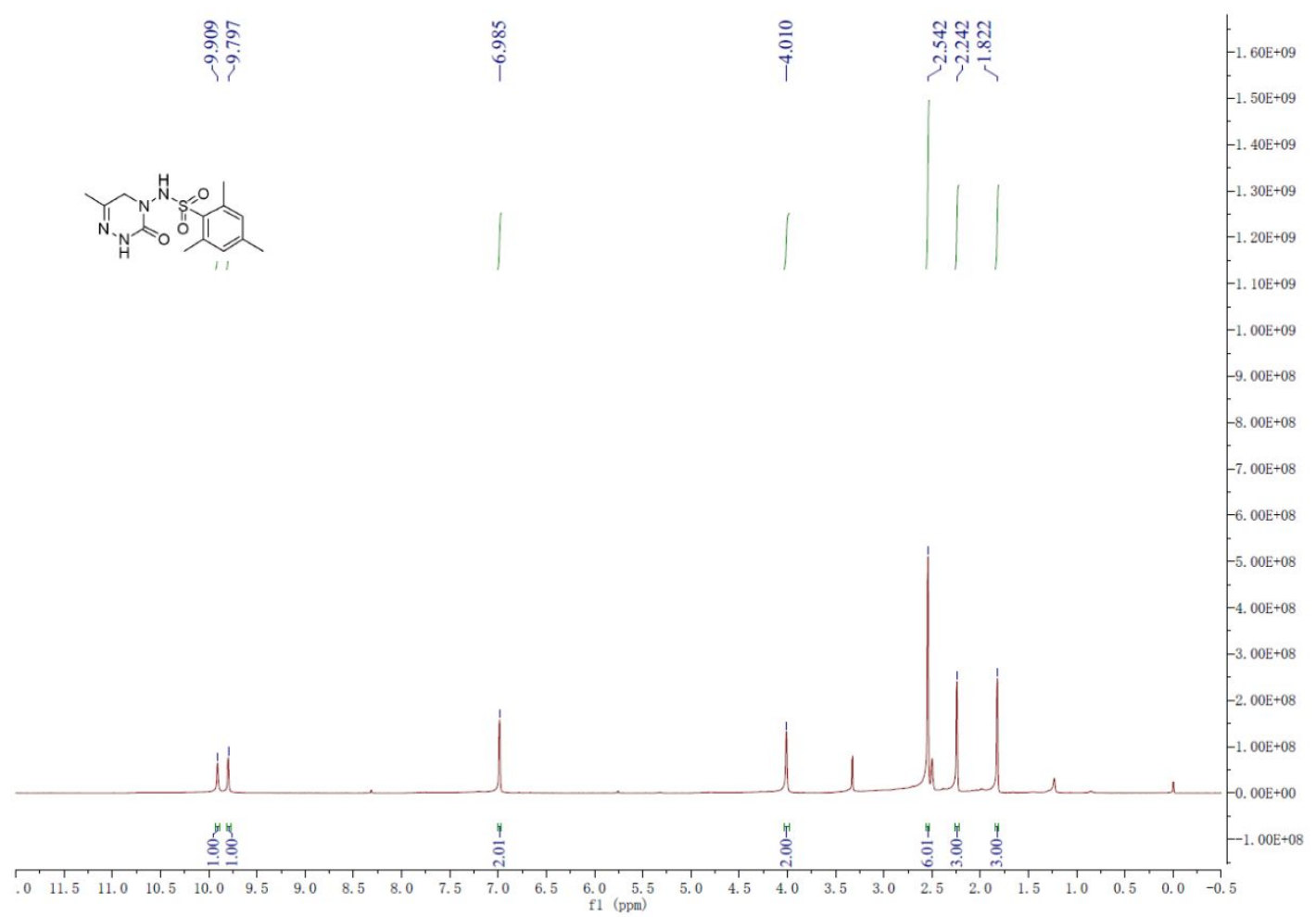

\section{I-12 ${ }^{13} \mathrm{C}$ NMR (100 MHz, DMSO- $\left.d_{6}\right)$}

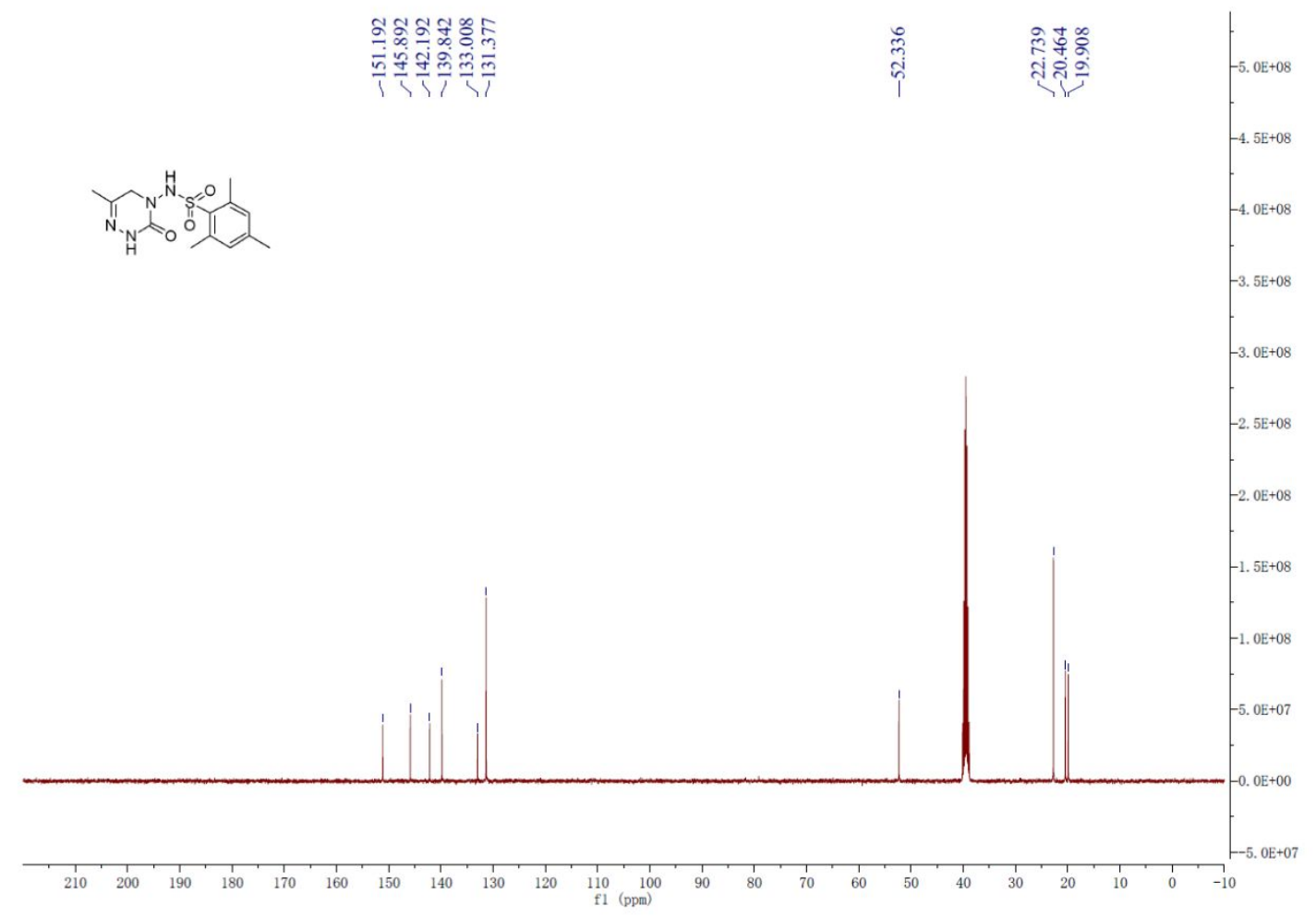


I-13 ${ }^{1} \mathrm{H}$ NMR (400 MHz, DMSO- $\left.d_{6}\right)$

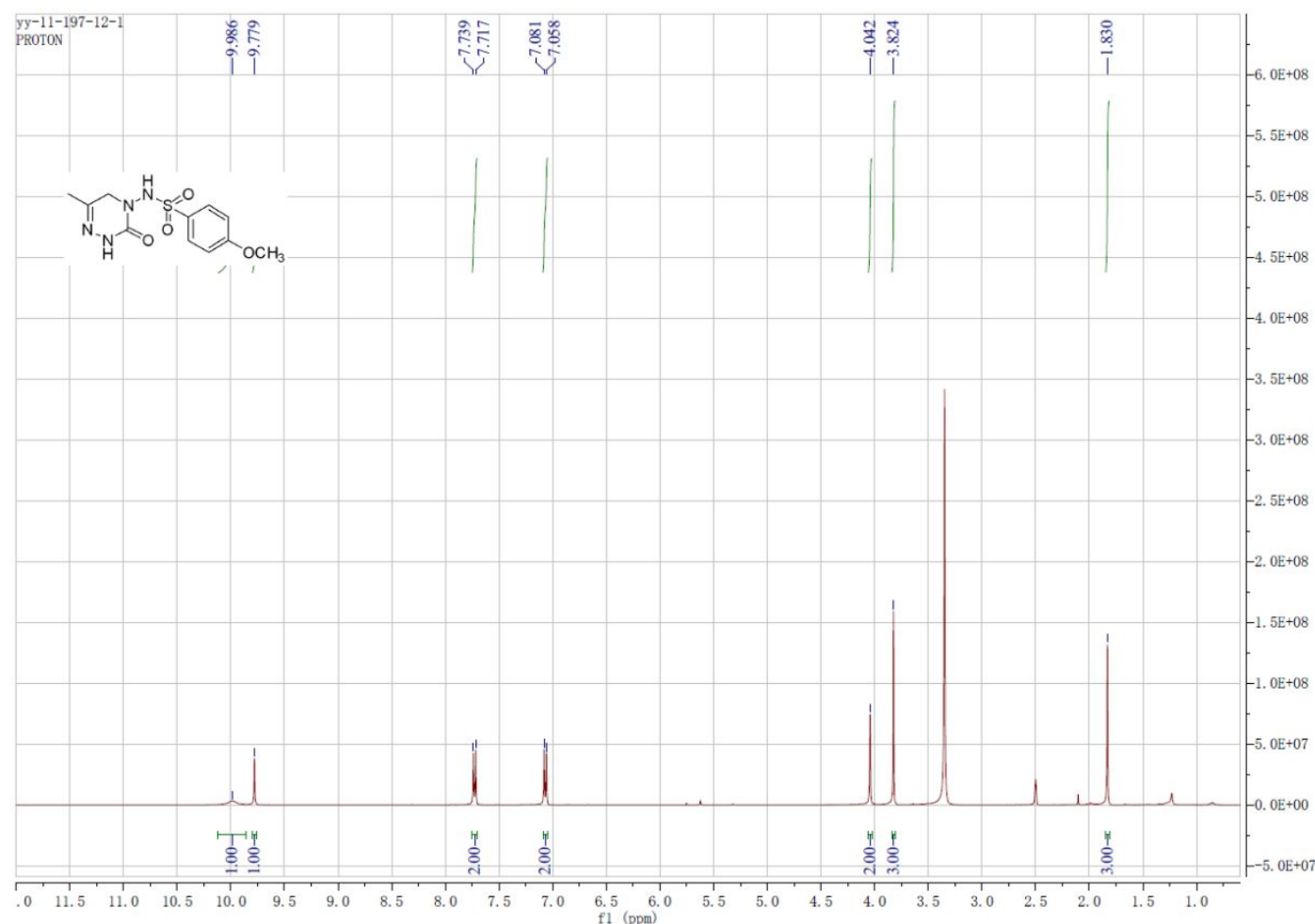

I-13 ${ }^{13} \mathrm{C}$ NMR (100 MHz, DMSO- $\left.d_{6}\right)$

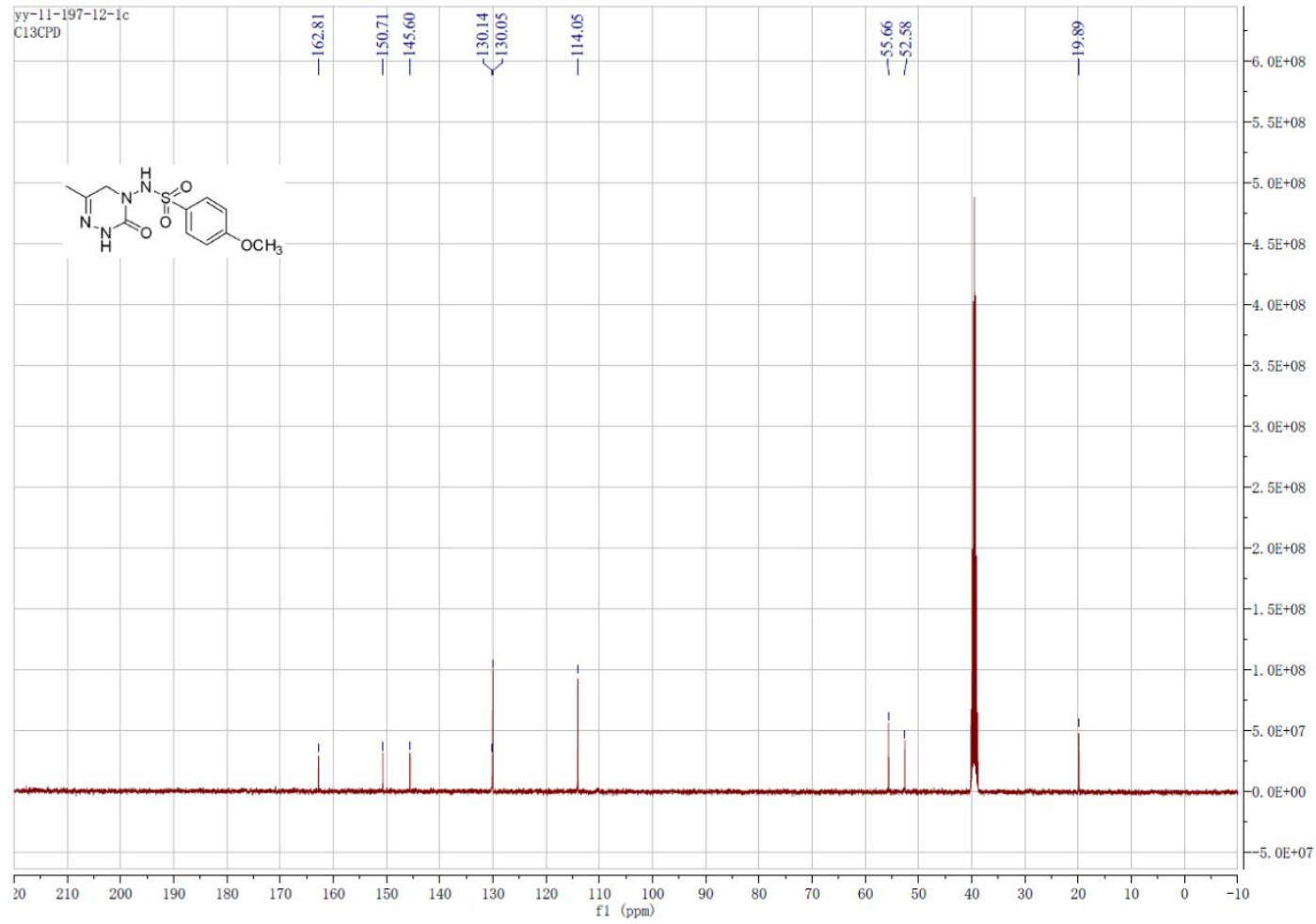


I-14 ${ }^{1} \mathrm{H}$ NMR (400 MHz, DMSO- $\left.d_{6}\right)$

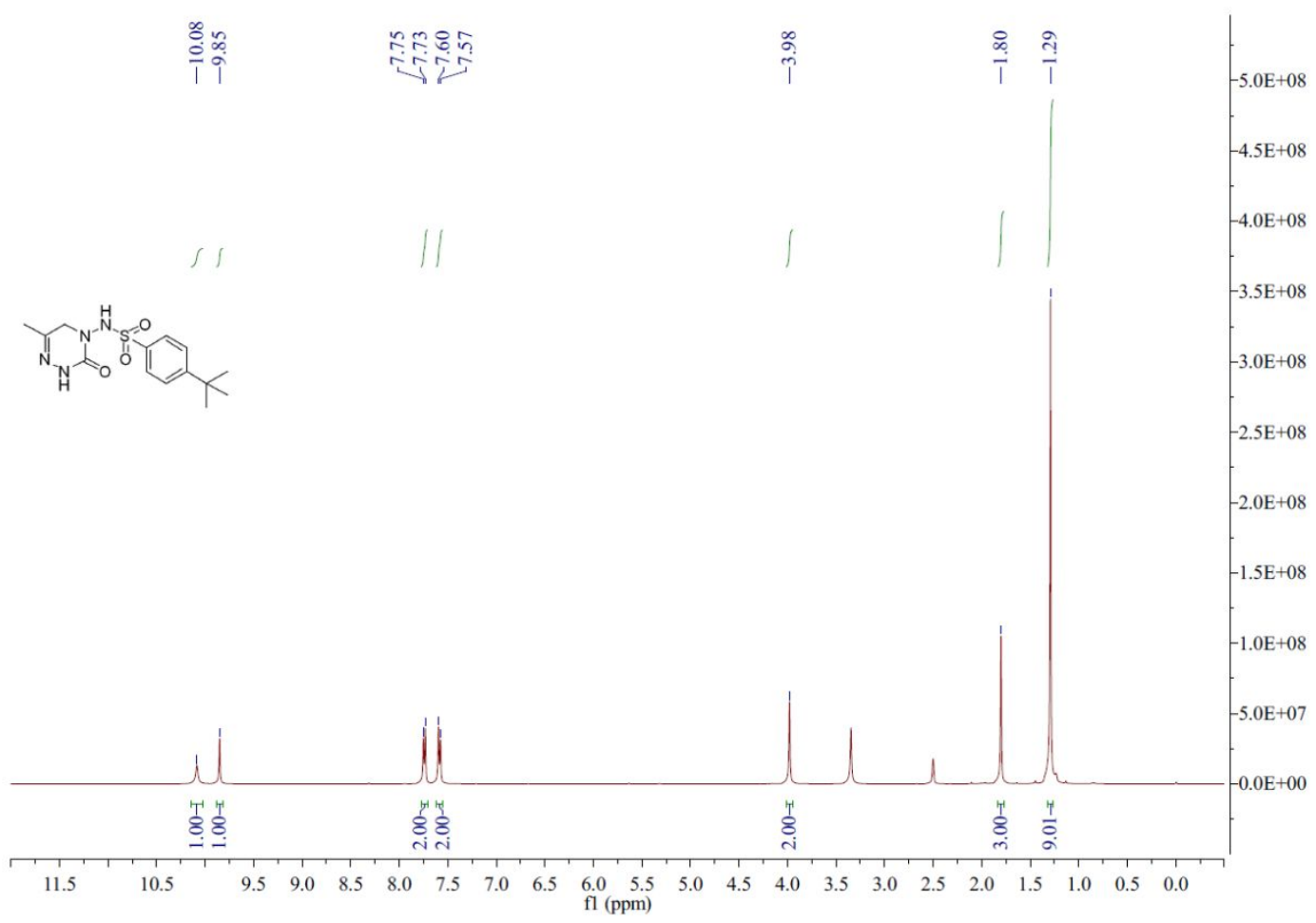

I-14 ${ }^{13} \mathrm{C}$ NMR (100 MHz, DMSO- $\left.d_{6}\right)$

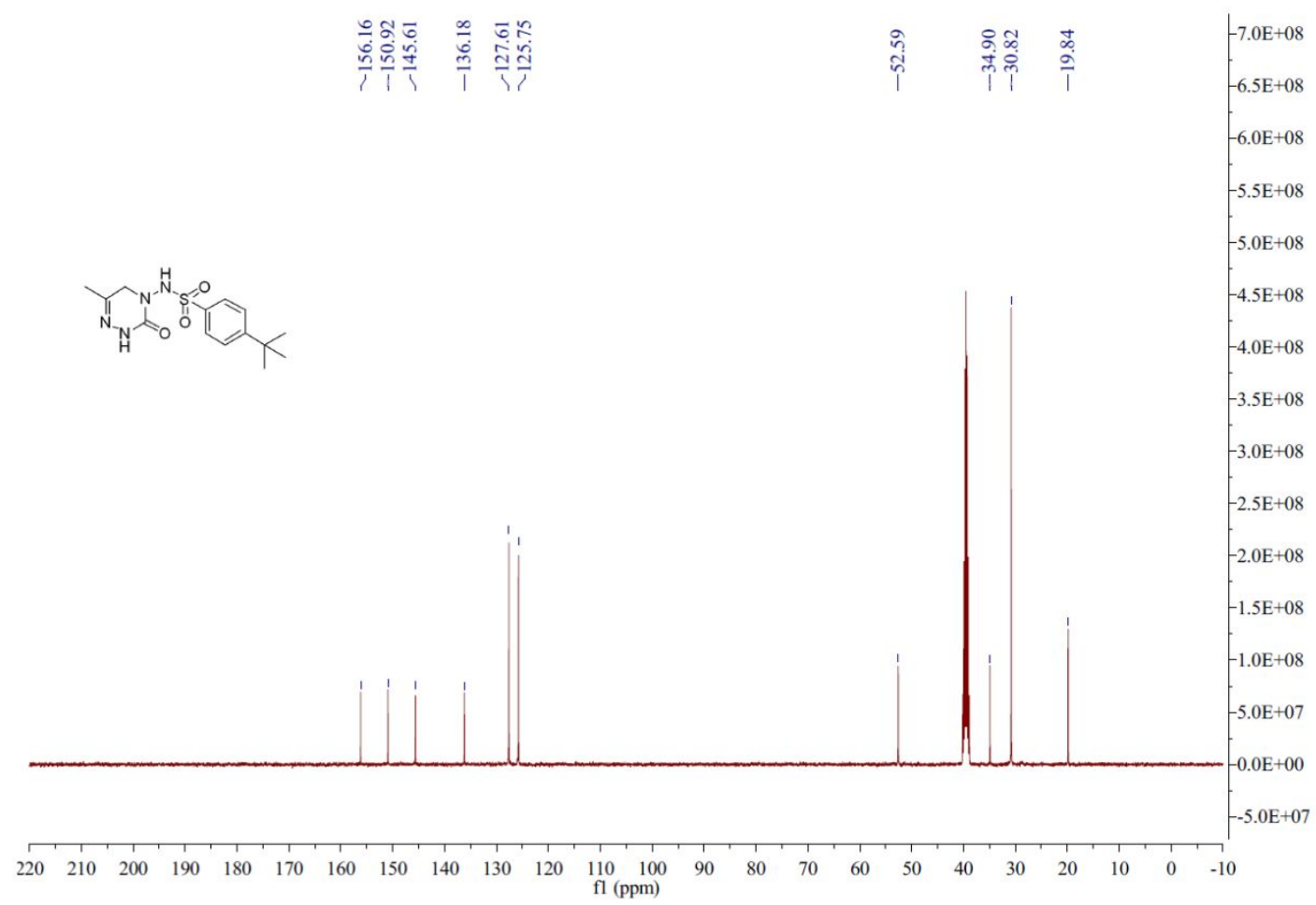


I-15 ${ }^{1} \mathrm{H}$ NMR (400 MHz, DMSO- $\left.d_{6}\right)$

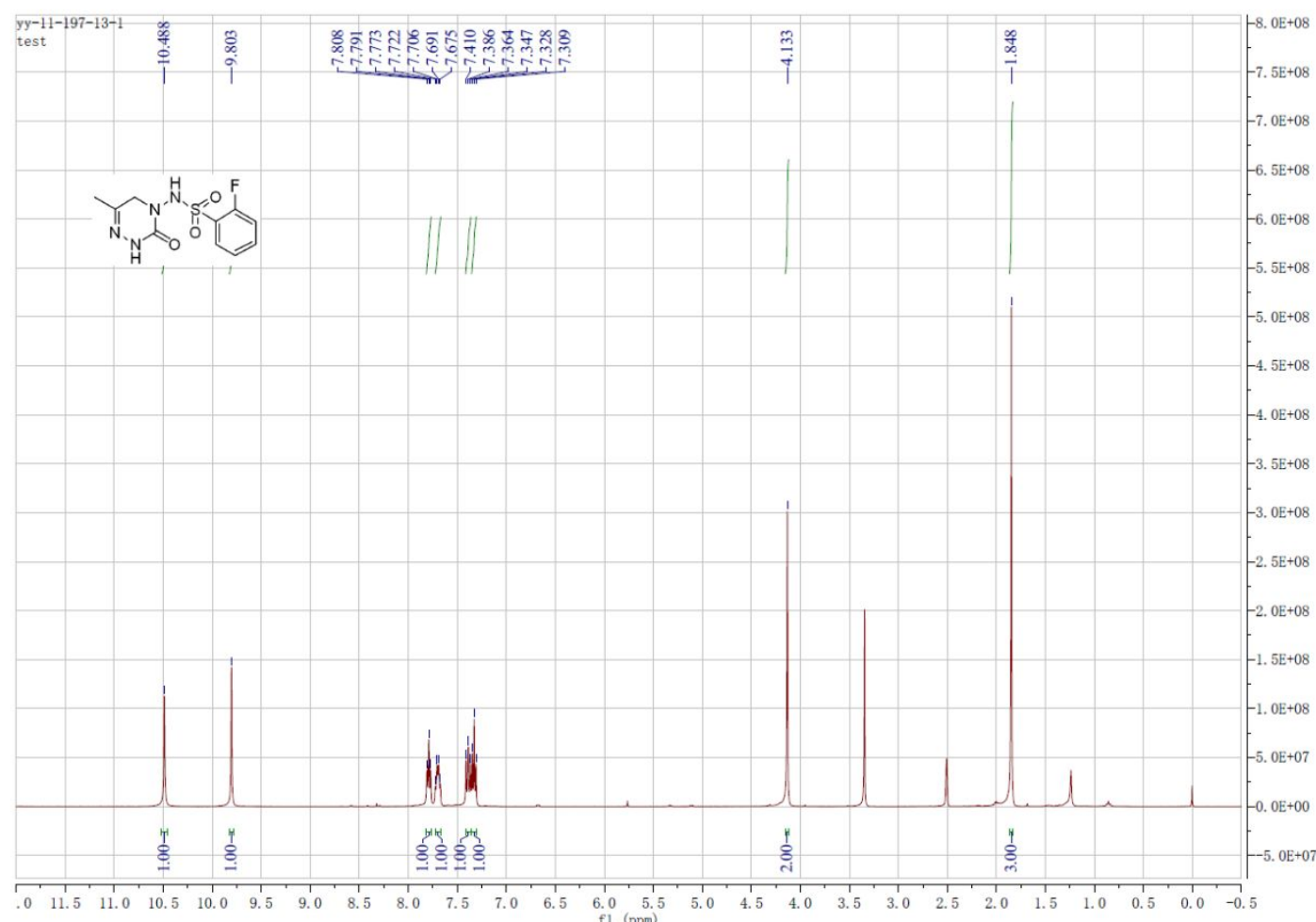

I-15 ${ }^{13} \mathrm{C}$ NMR (100 MHz, DMSO- $\left.d_{6}\right)$

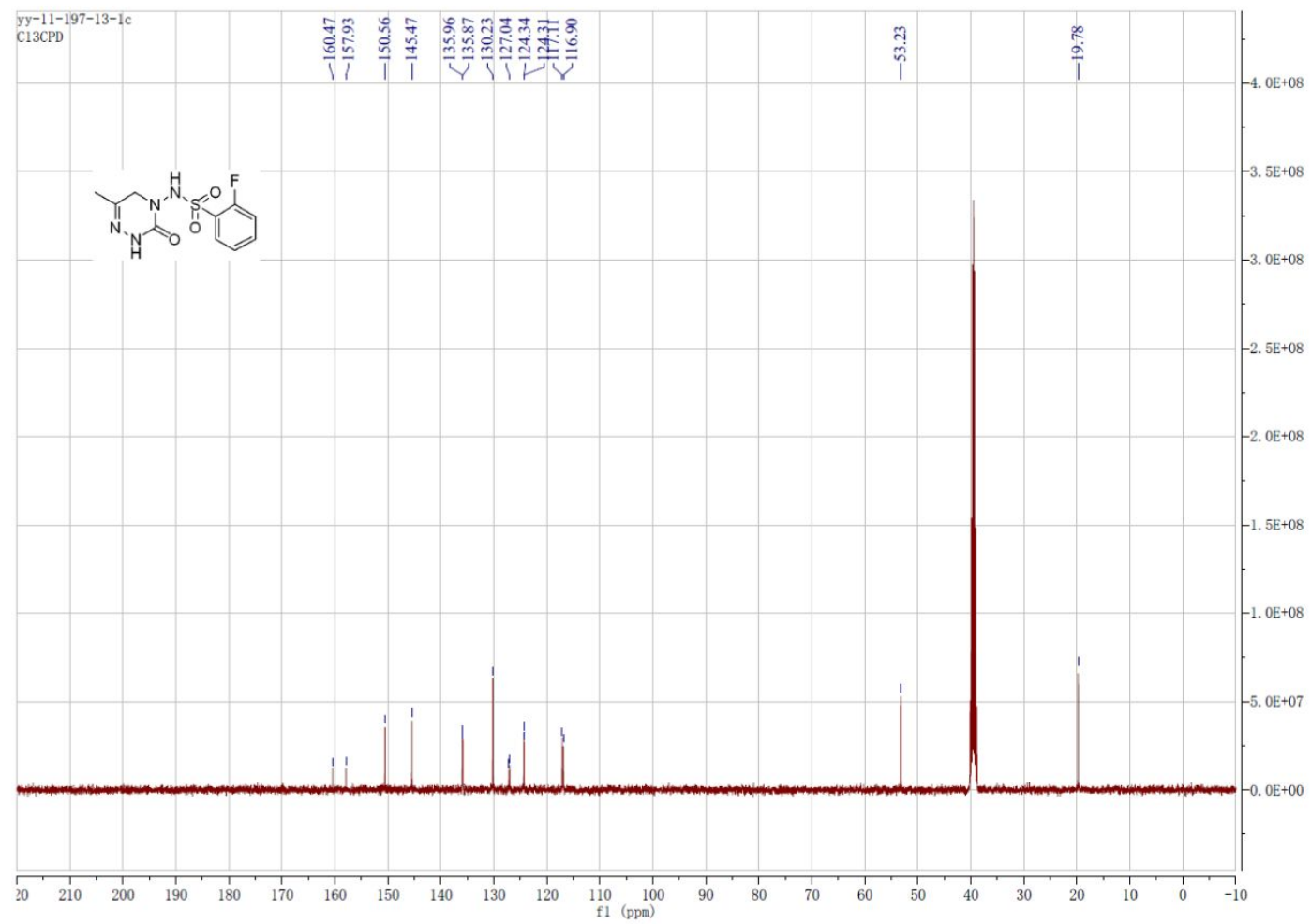


I-16 ${ }^{1} \mathrm{H}$ NMR (400 MHz, DMSO- $\left.d_{6}\right)$

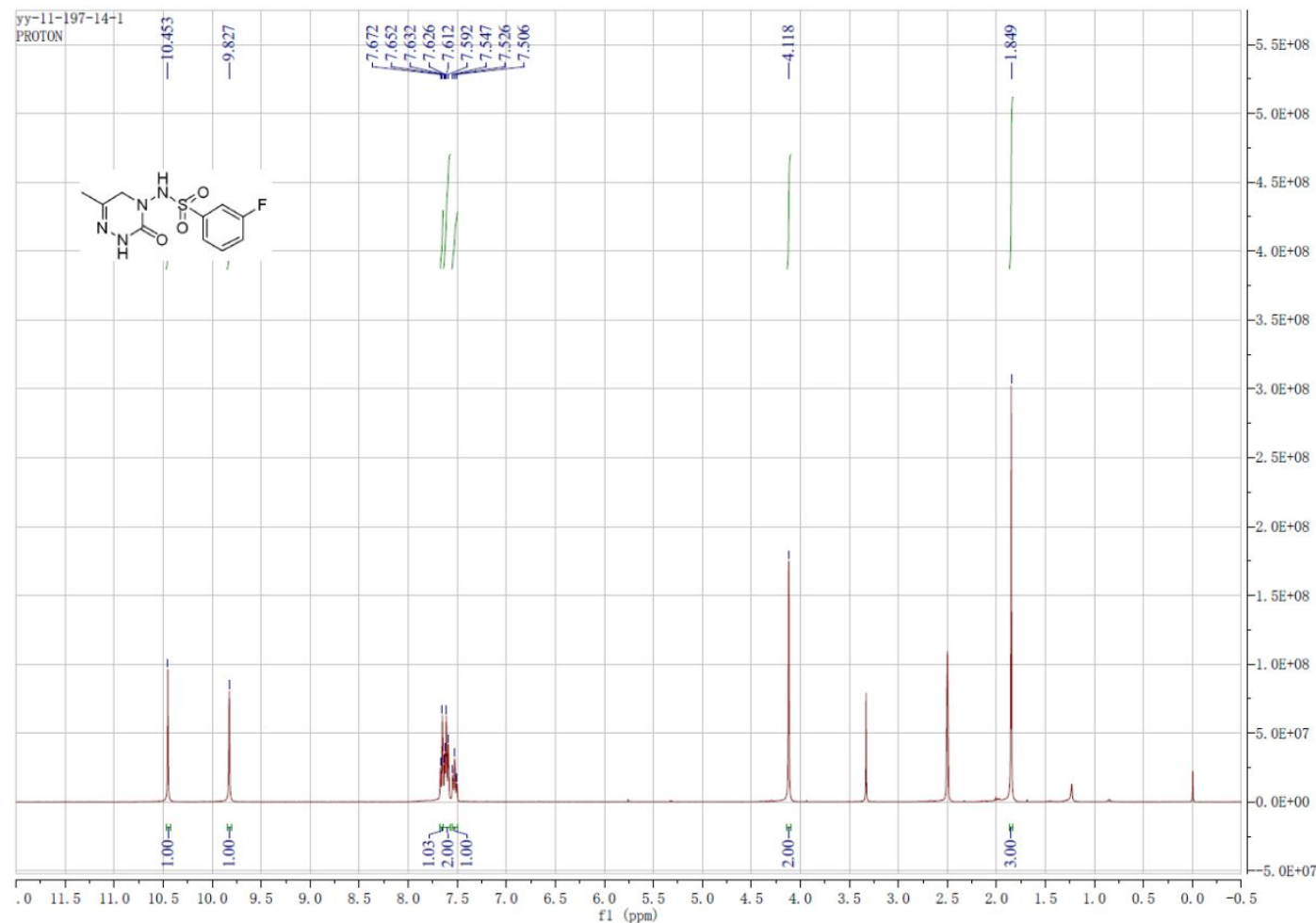

\section{I-16 ${ }^{13} \mathrm{C}$ NMR (100 MHz, DMSO- $\left.d_{6}\right)$}

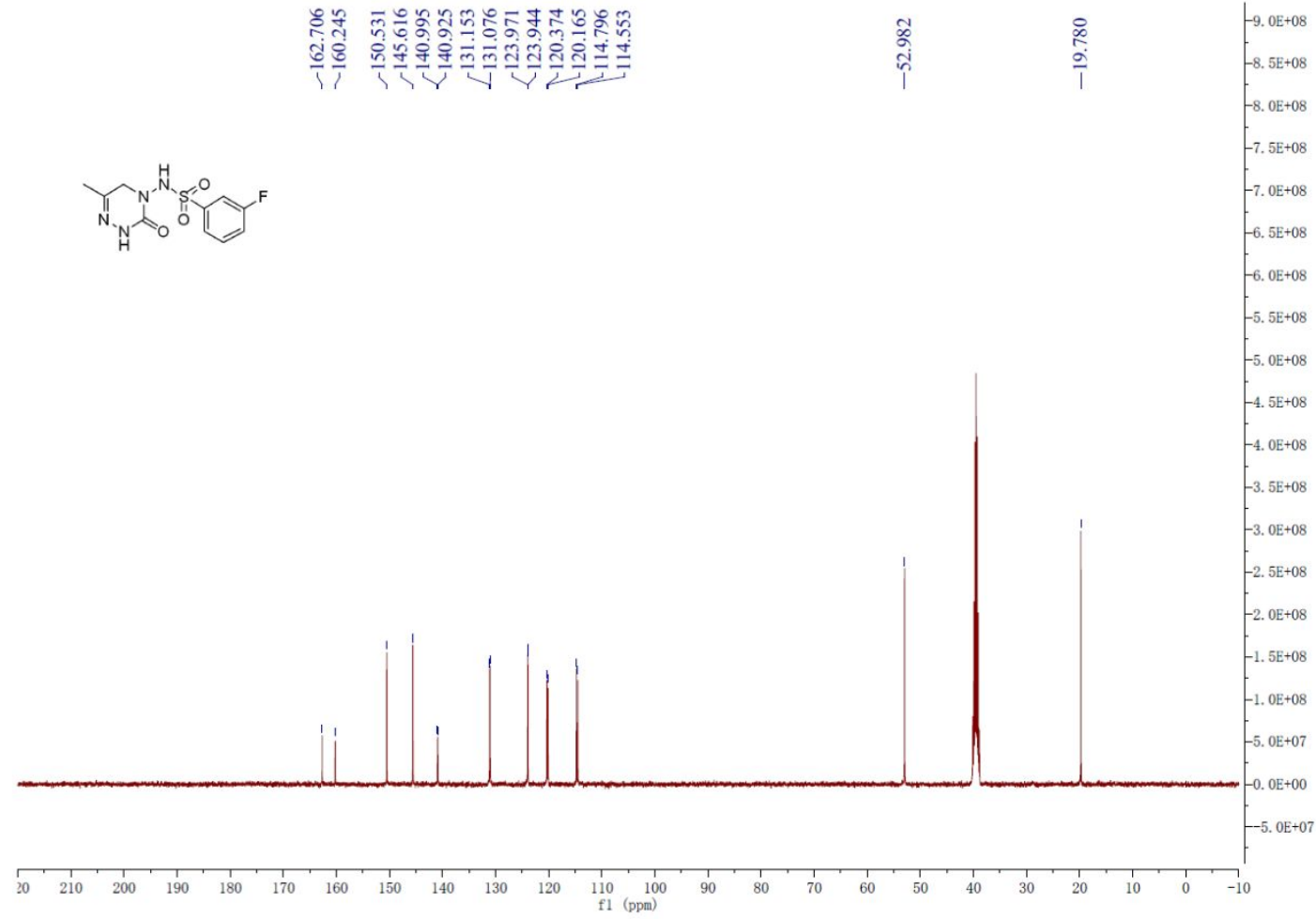


I-17 ${ }^{1} \mathrm{H}$ NMR (400 MHz, DMSO- $\left.d_{6}\right)$

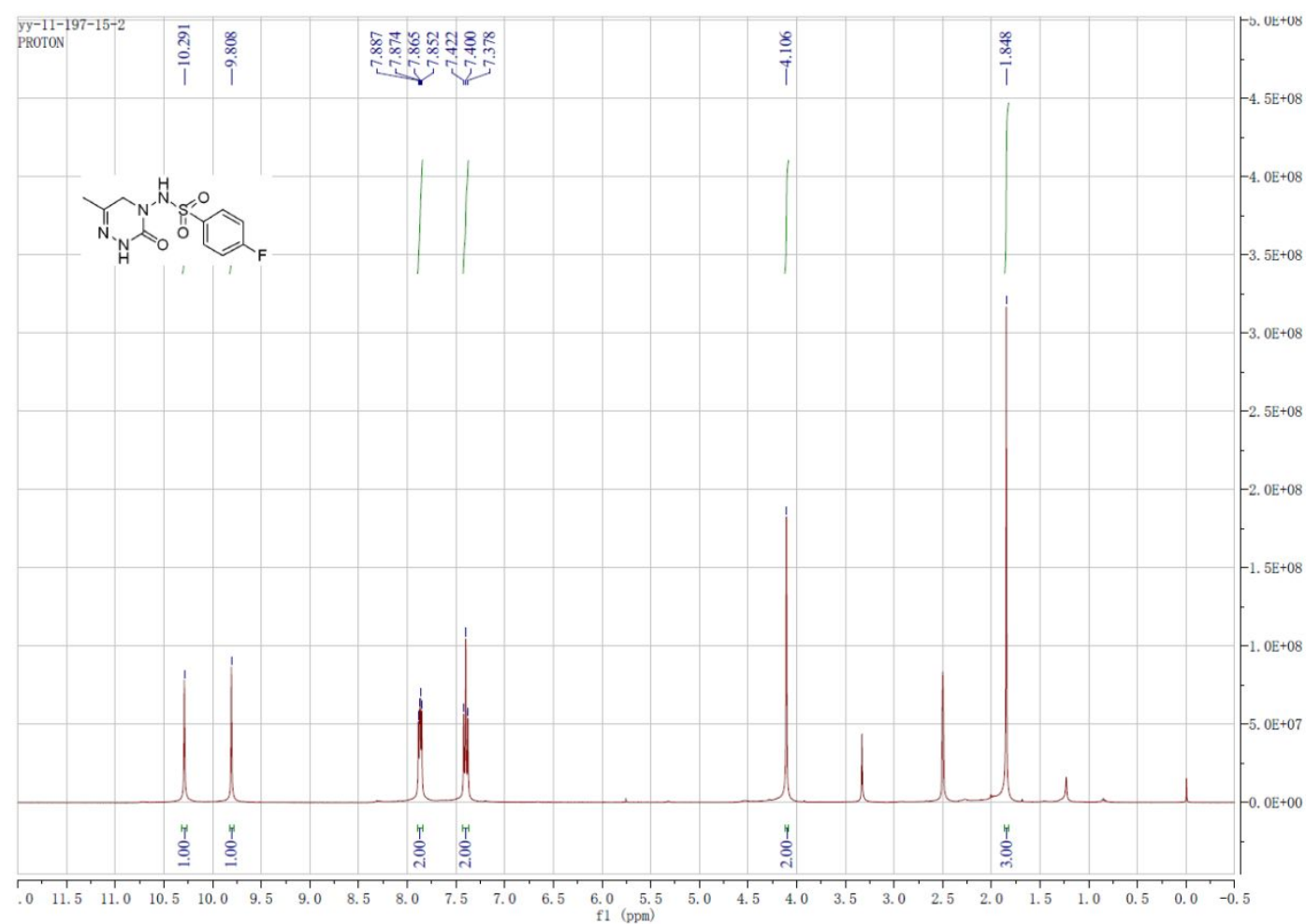

I-17 ${ }^{13} \mathrm{C}$ NMR (100 MHz, DMSO- $\left.d_{6}\right)$

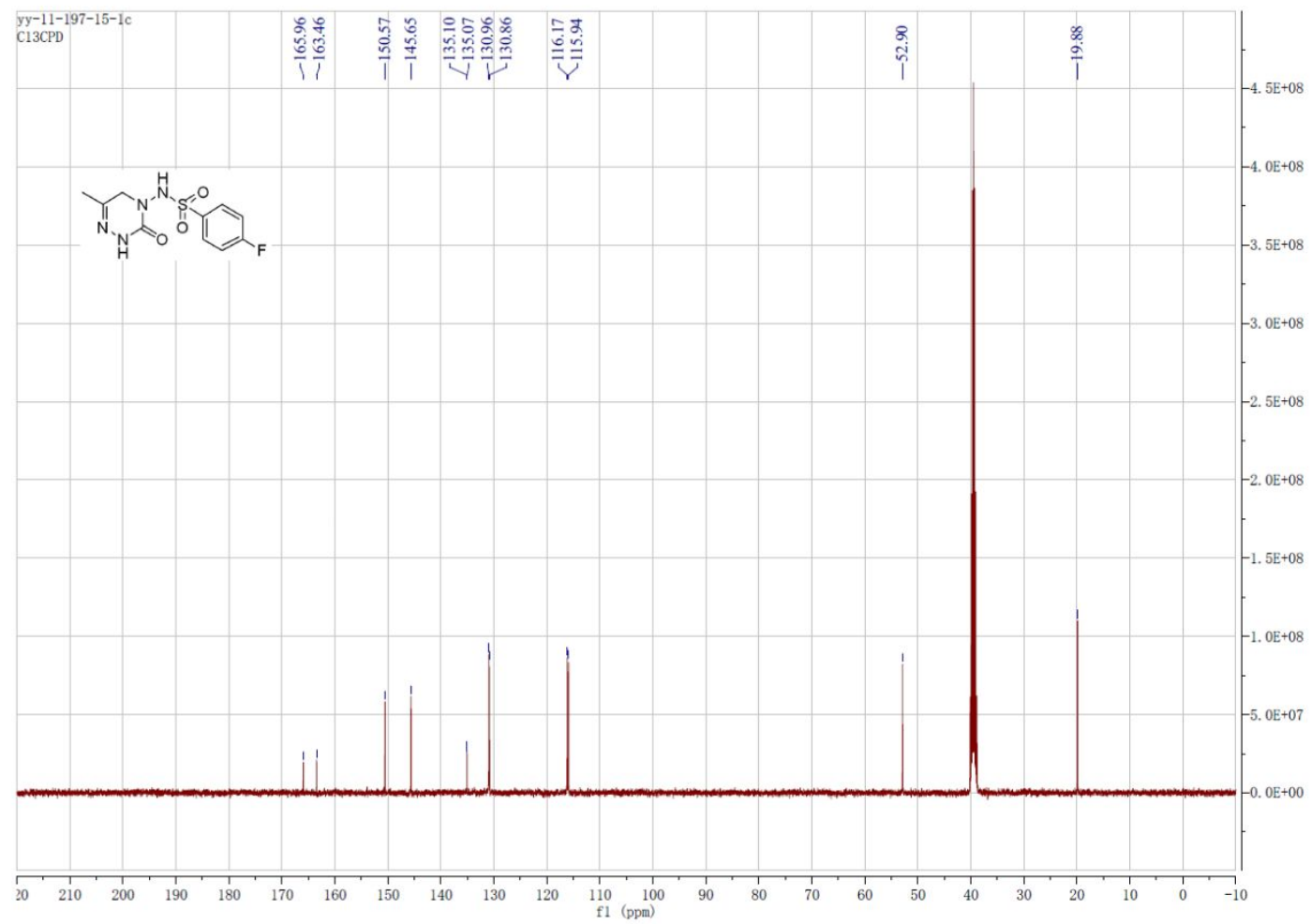


I-18 ${ }^{1} \mathrm{H}$ NMR (400 MHz, DMSO- $\left.d_{6}\right)$

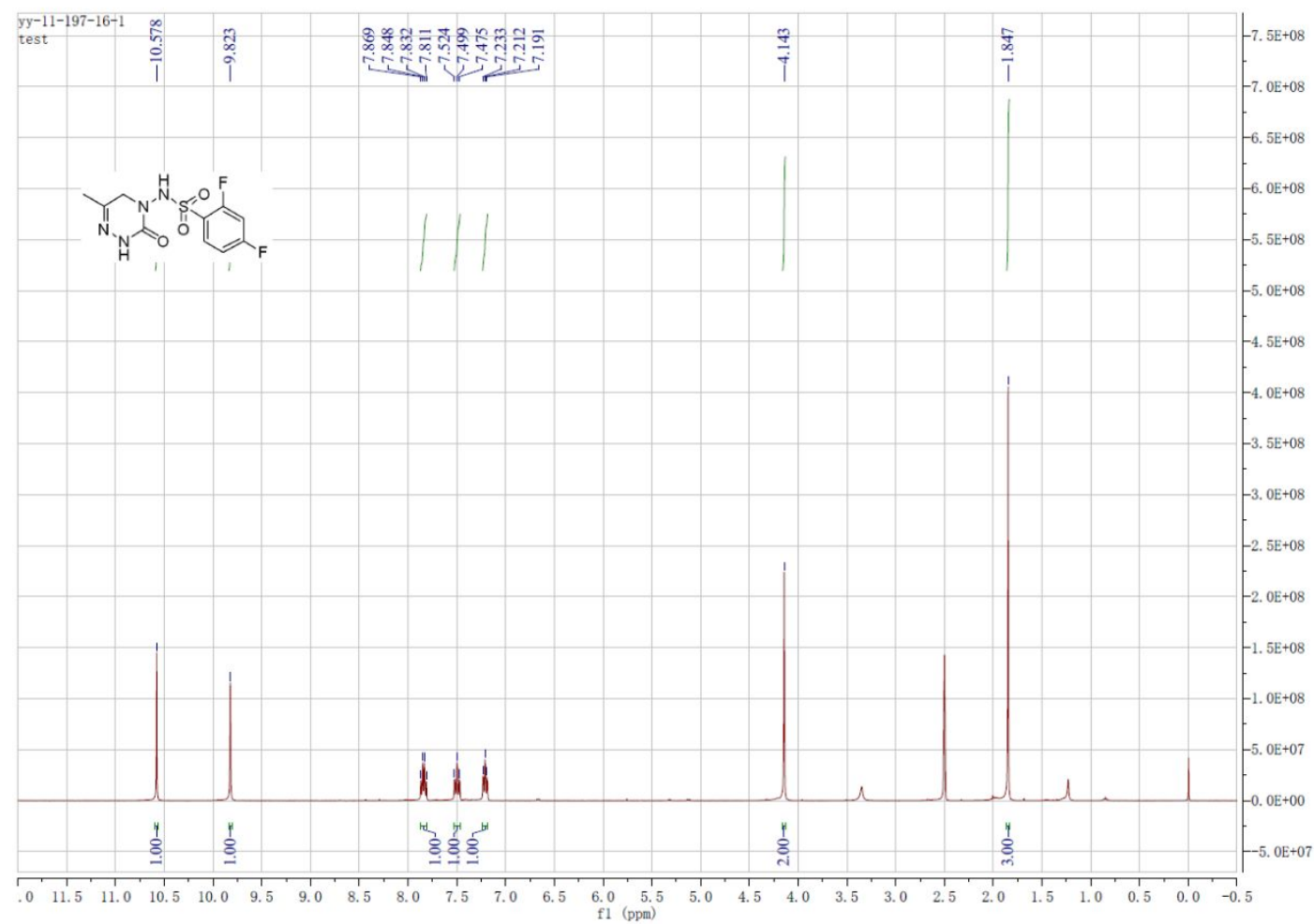

I-18 ${ }^{13} \mathrm{C}$ NMR (100 MHz, DMSO- $\left.d_{6}\right)$

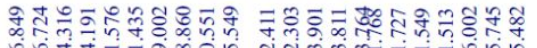

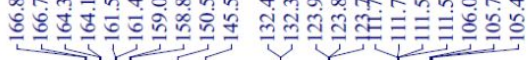

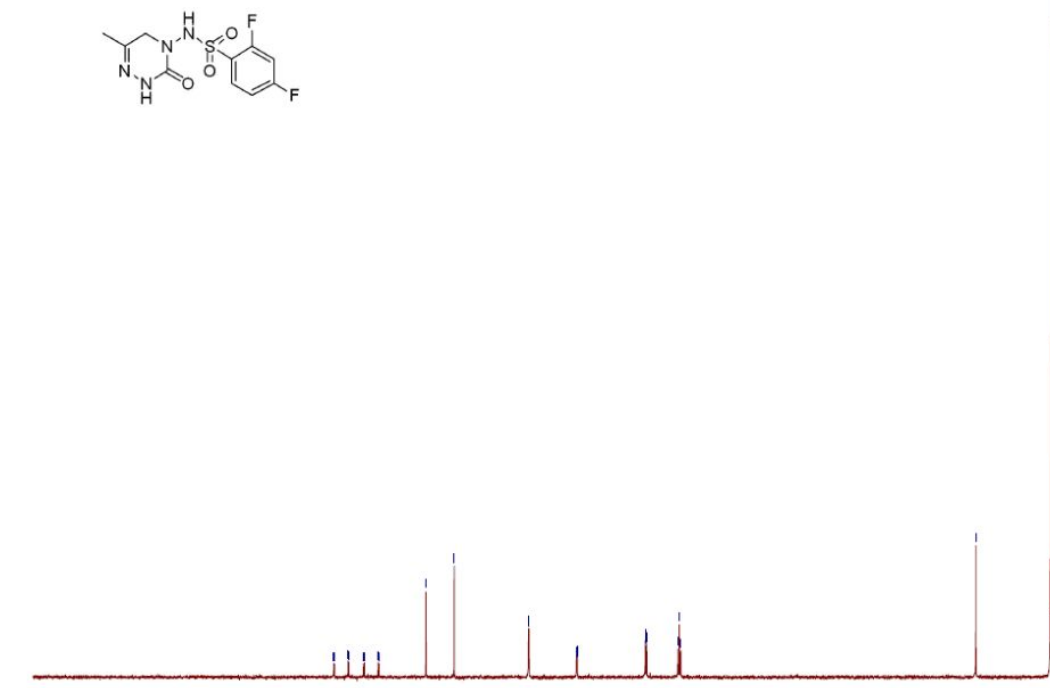

㝦

$-3.4 \mathrm{E}+08$

3. $2 \mathrm{E}+08$

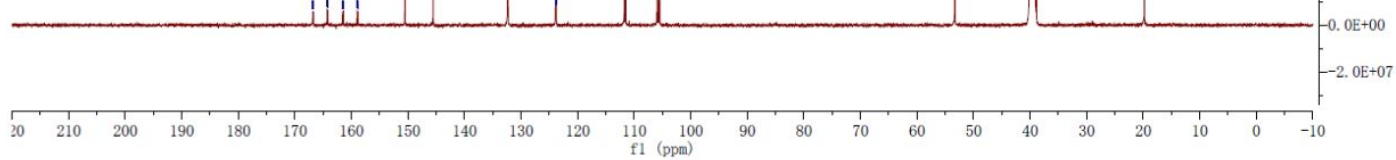


I-19 ${ }^{1} \mathrm{H}$ NMR $\left(400 \mathrm{MHz}, \mathrm{DMSO}-d_{6}\right)$

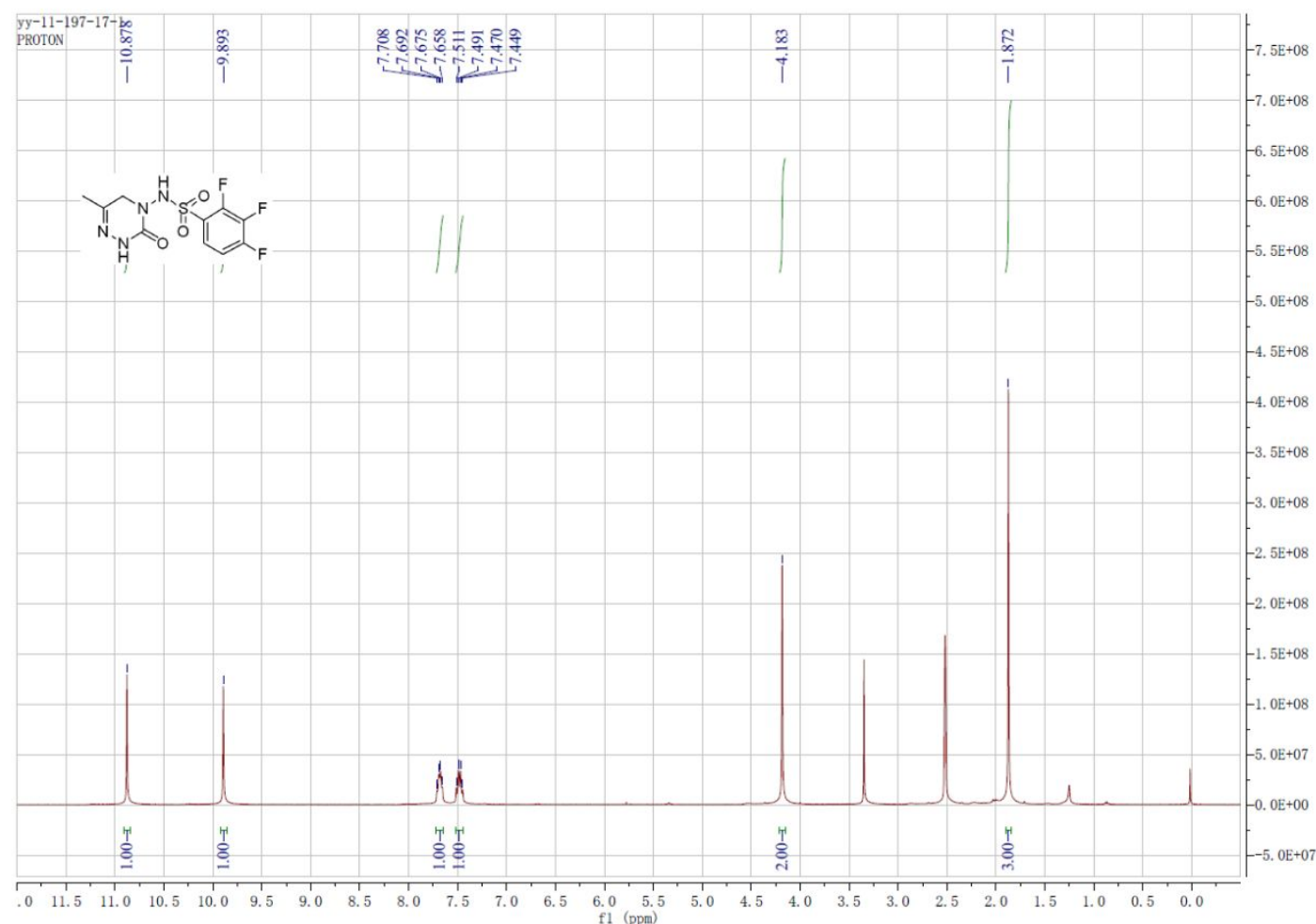

\section{I-19 ${ }^{13} \mathrm{C}$ NMR (100 MHz, DMSO- $\left.d_{6}\right)$}

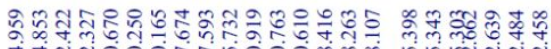

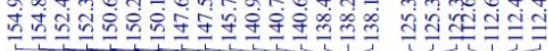

管

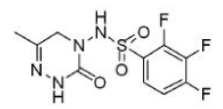

ڤี

$\underset{\substack{n \\ \frac{\infty}{j}}}{\stackrel{n}{i}}$

4. $5 \mathrm{E}+08$

$0 \mathrm{E}+08$

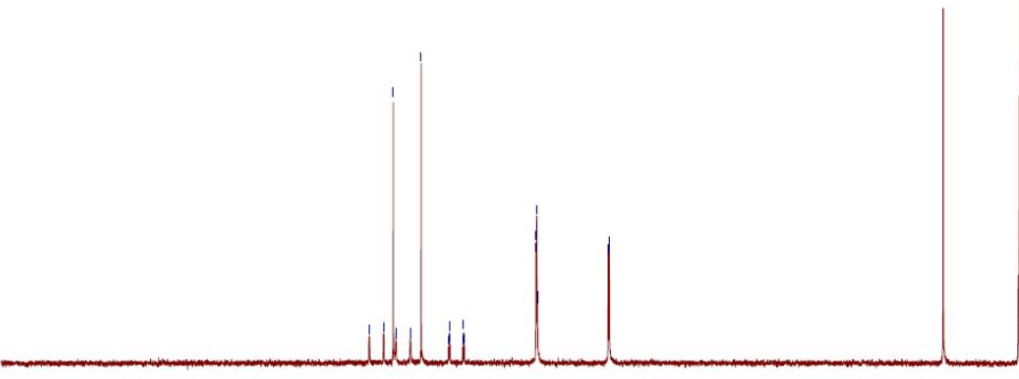




\section{I-20 ${ }^{1} \mathrm{H}$ NMR (400 MHz, DMSO- $\left.d_{6}\right)$}

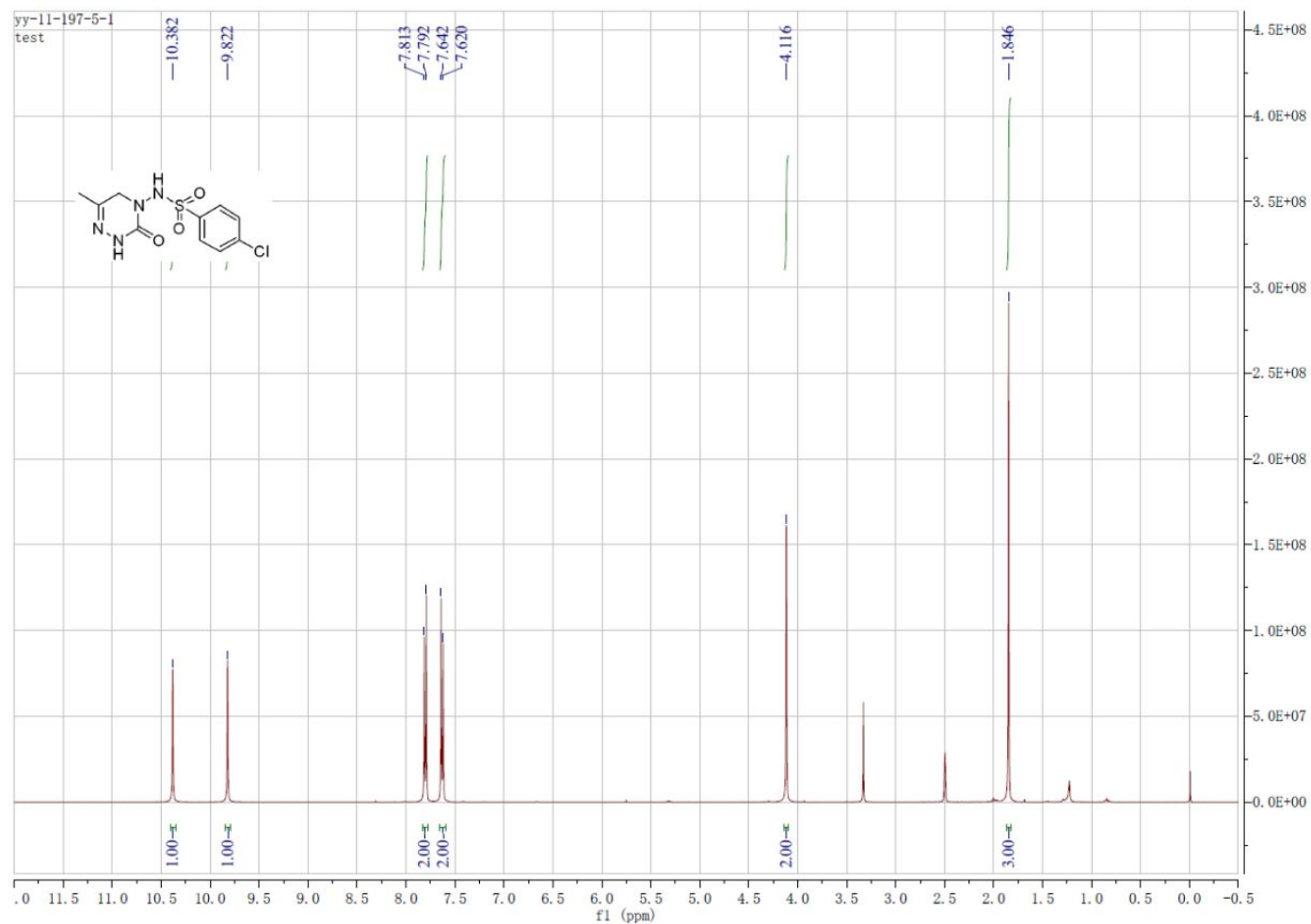

\section{I-20 ${ }^{13} \mathrm{C}$ NMR (100 MHz, DMSO- $\left.d_{6}\right)$}

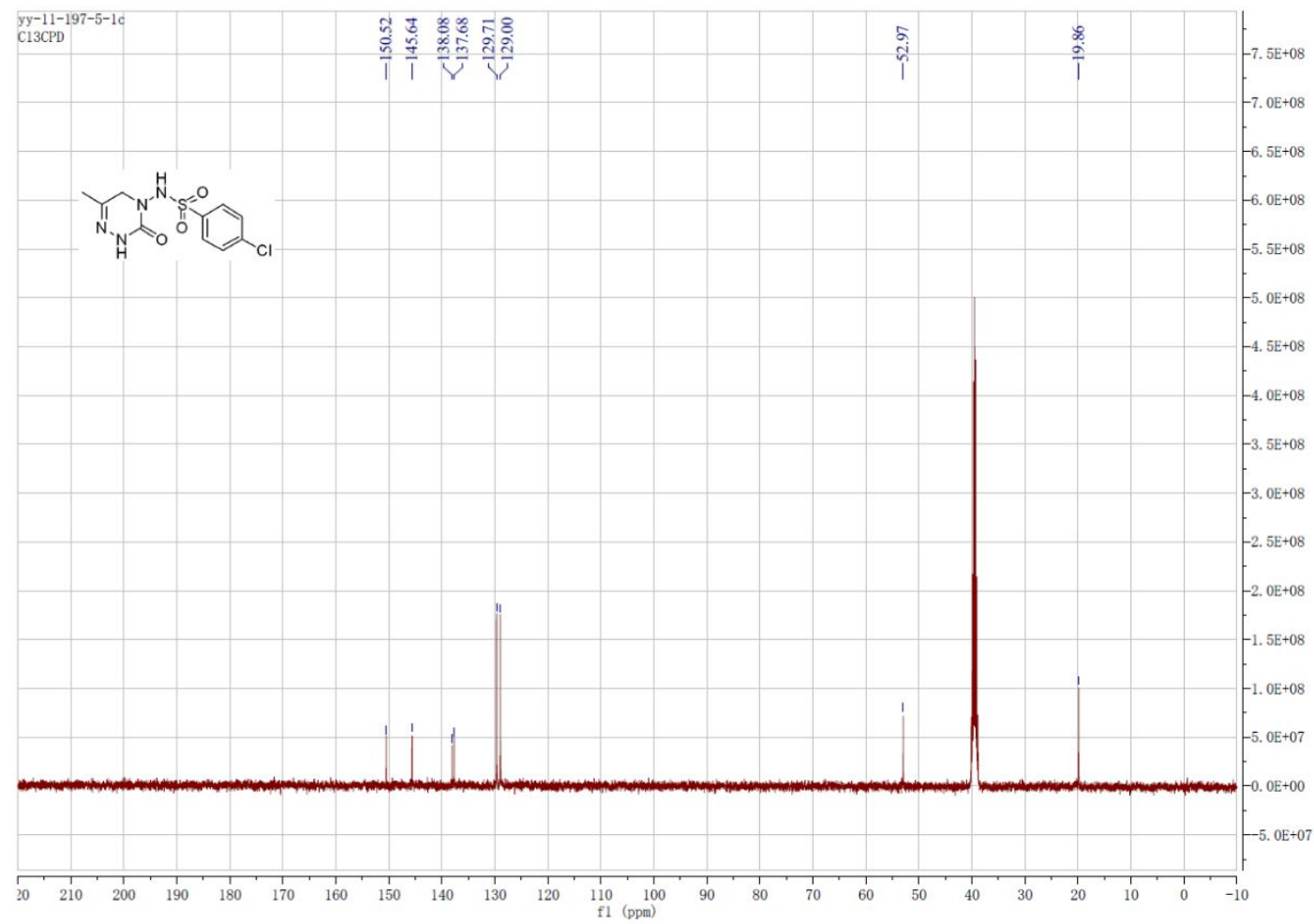


I-21 ${ }^{1} \mathrm{H}$ NMR (400 MHz, DMSO- $\left.d_{6}\right)$

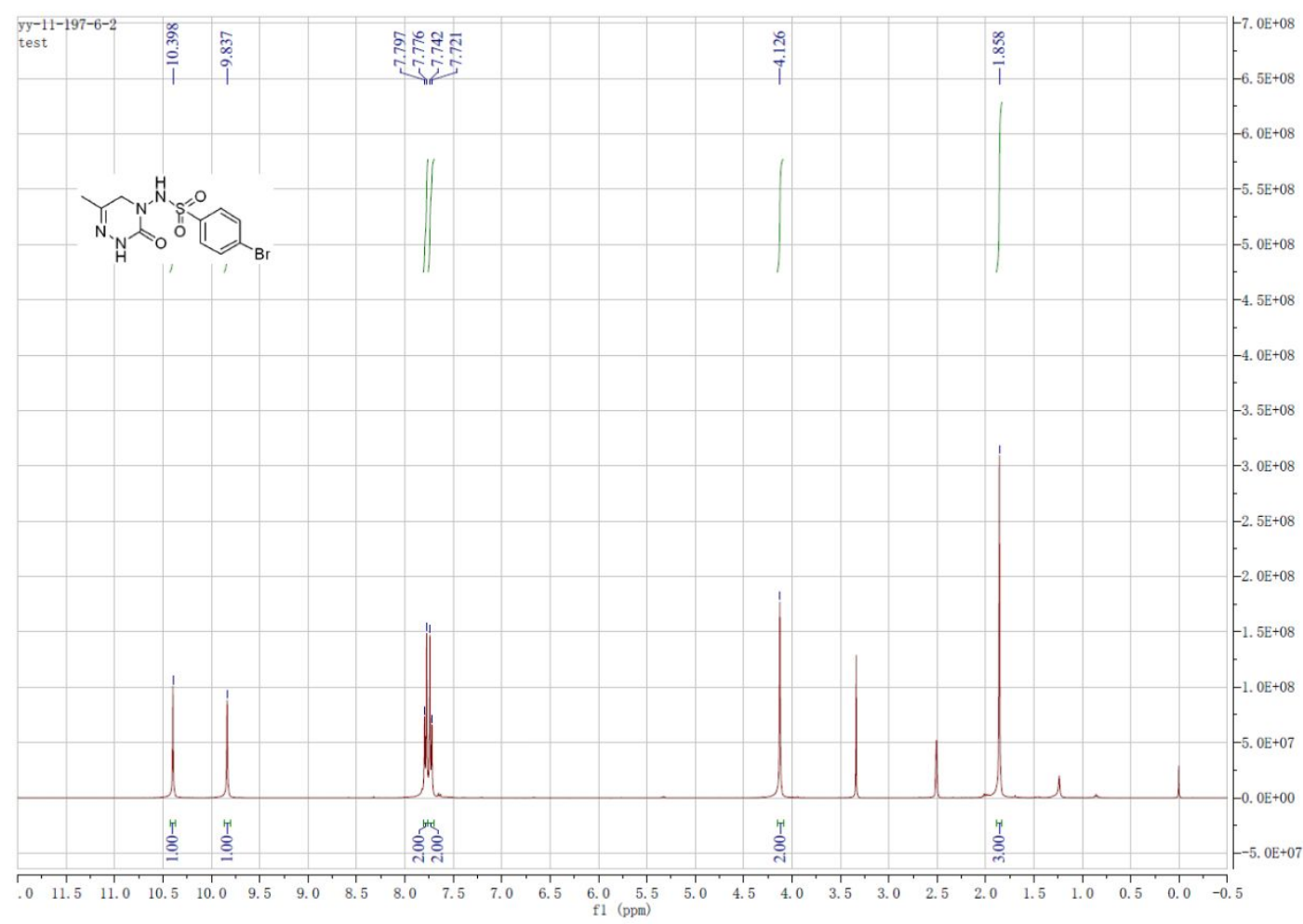

I-21 ${ }^{13} \mathrm{C}$ NMR (100 MHz, DMSO- $\left.d_{6}\right)$

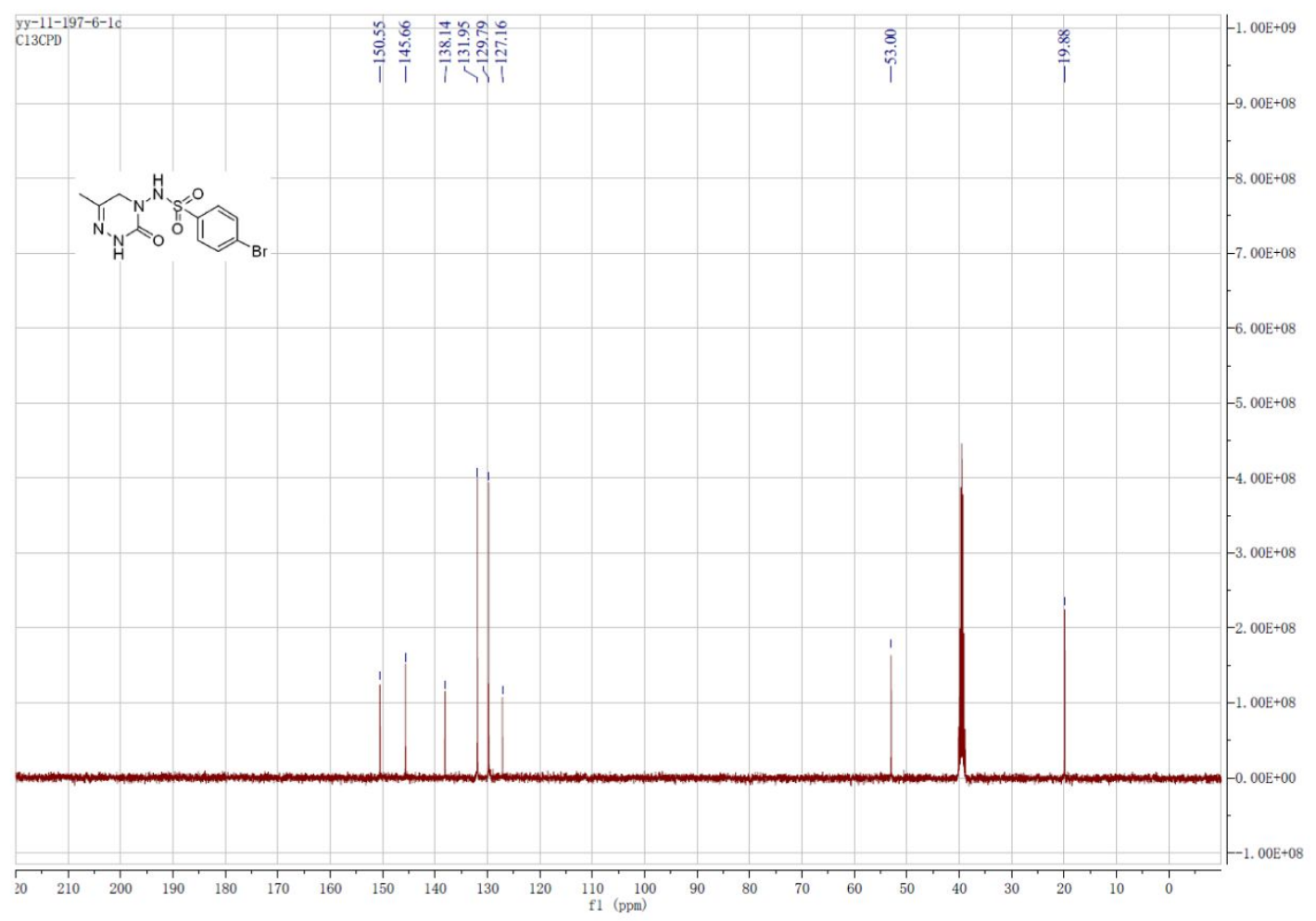


I-22 ${ }^{1} \mathrm{H}$ NMR (400 MHz, DMSO- $d_{6}$ )

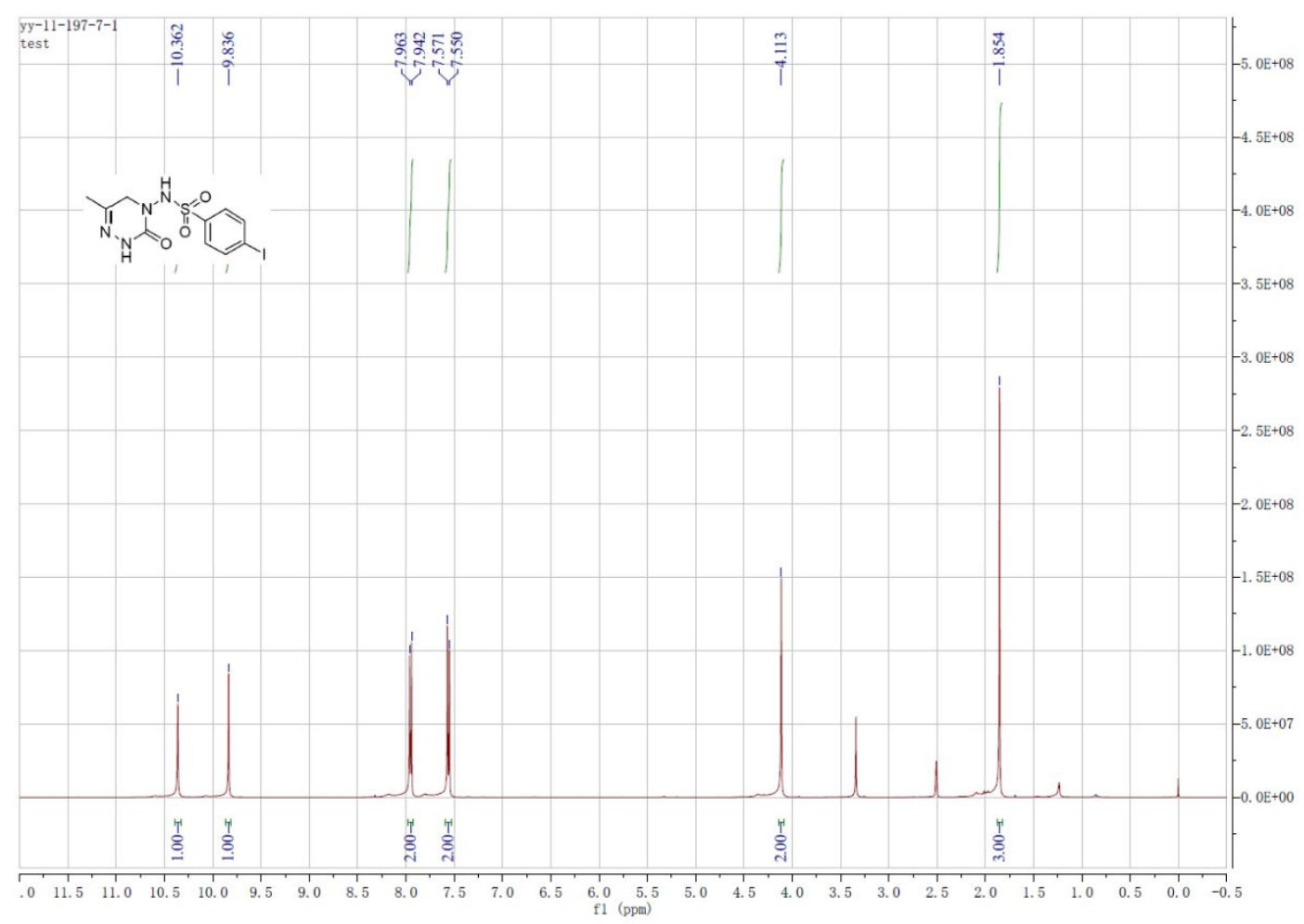

I-22 ${ }^{13} \mathrm{C}$ NMR (100 MHz, DMSO- $\left.d_{6}\right)$

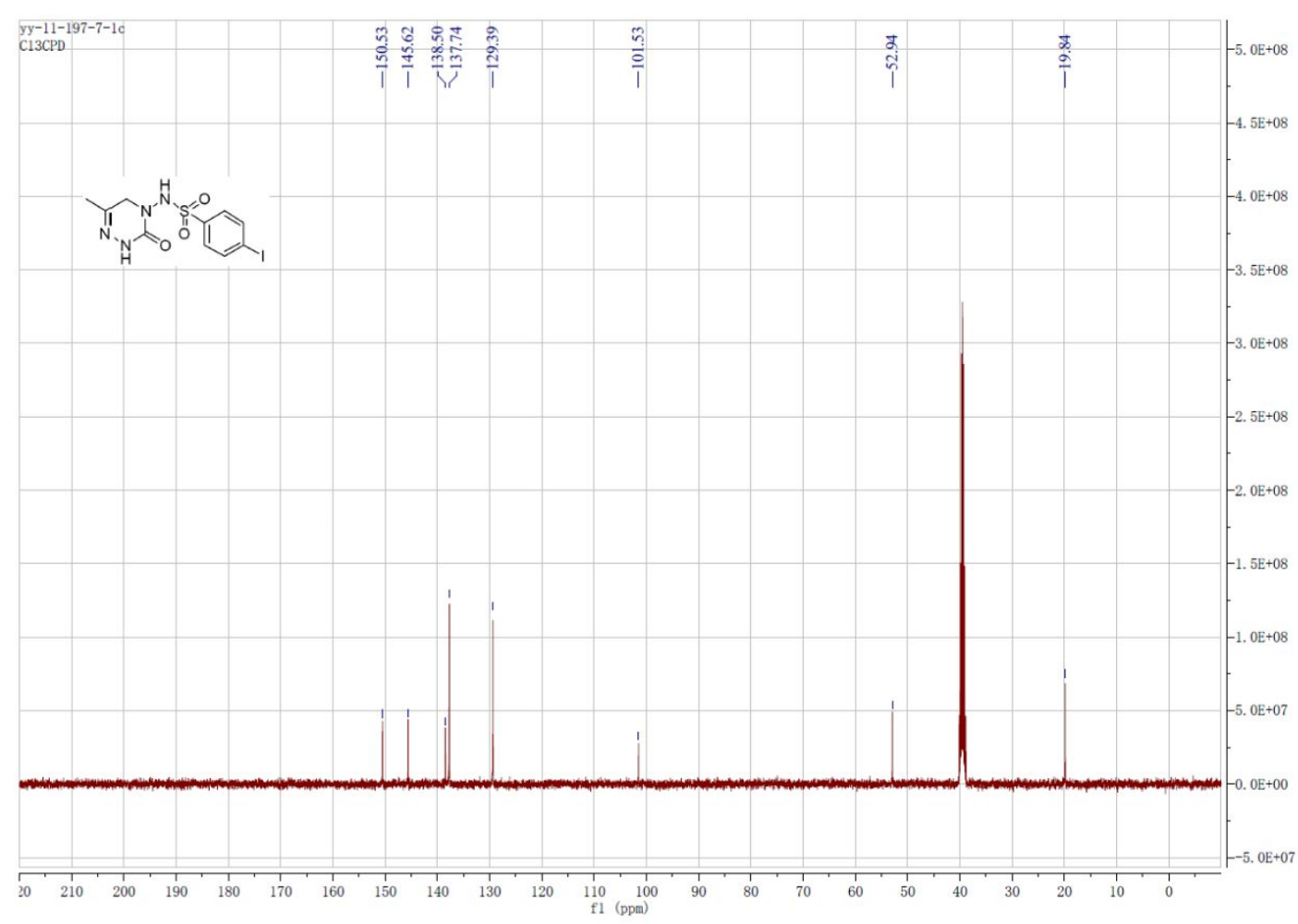


I-23 ${ }^{1} \mathrm{H}$ NMR (300 MHz, DMSO- $\left.d_{6}\right)$

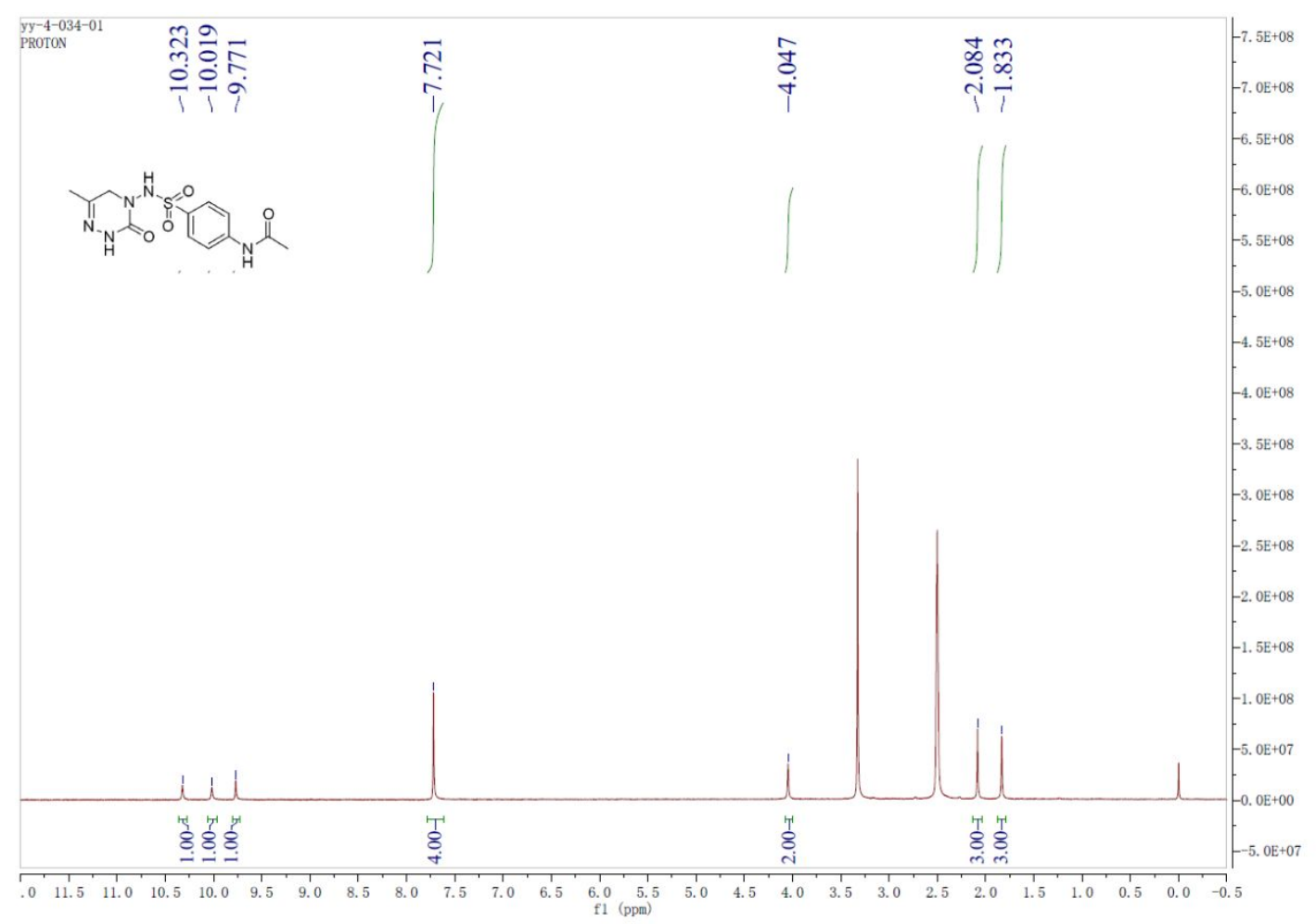

I-23 ${ }^{13} \mathrm{C}$ NMR (100 MHz, DMSO- $\left.d_{6}\right)$

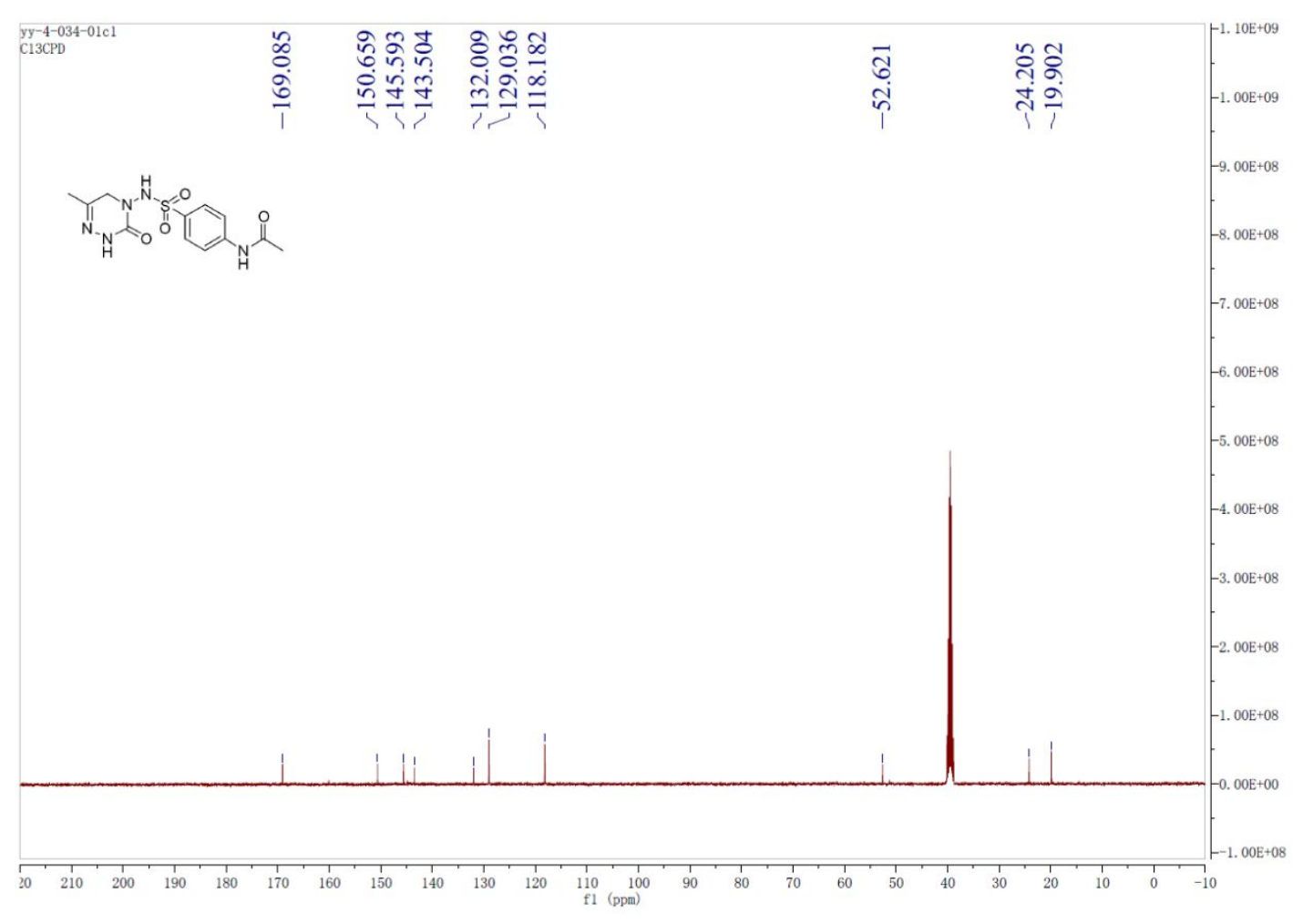




\section{I-24 ${ }^{1} \mathrm{H}$ NMR (400 MHz, DMSO- $\left.d_{6}\right)$}

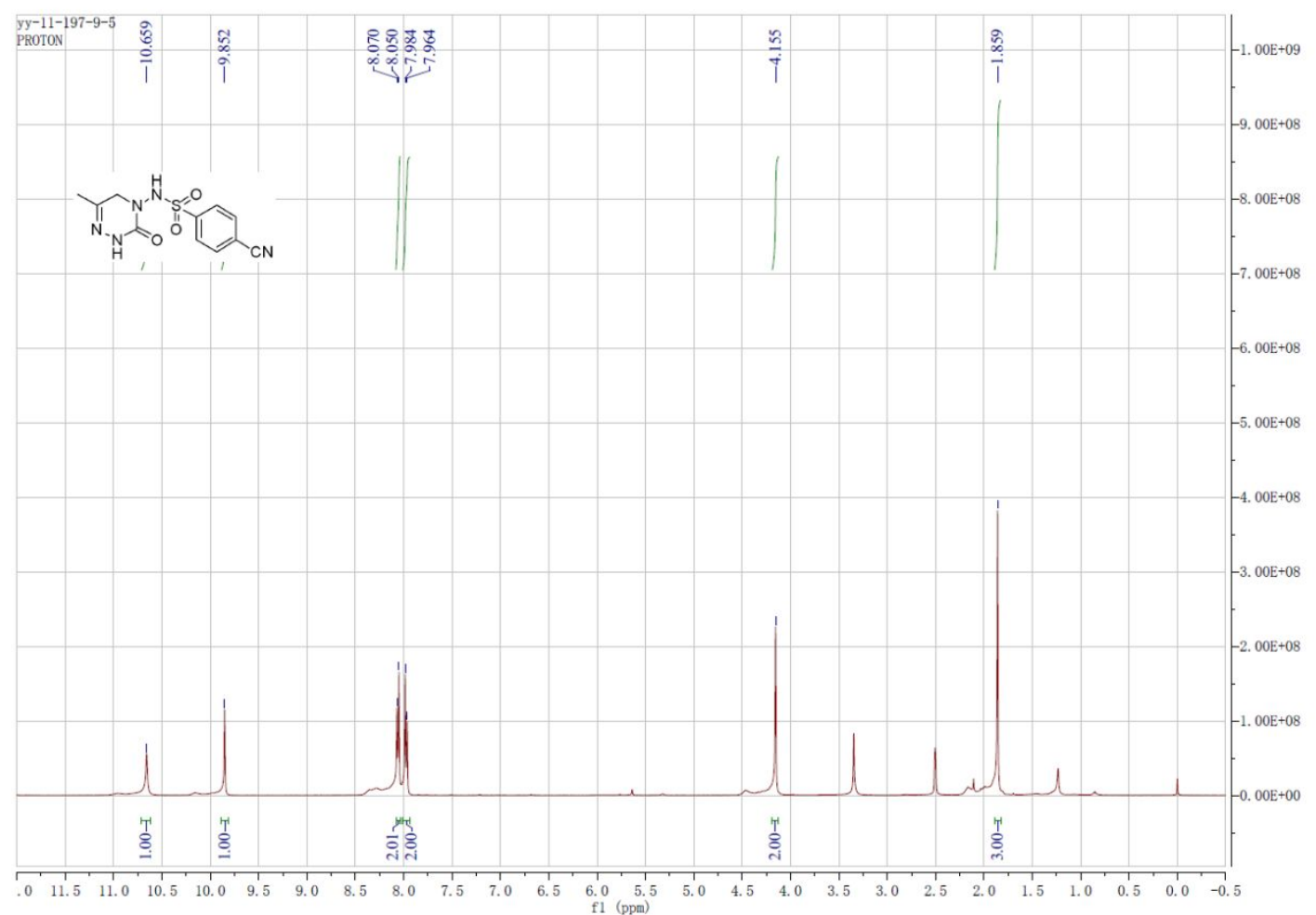

I-24 ${ }^{13} \mathrm{C}$ NMR (100 MHz, DMSO- $\left.d_{6}\right)$

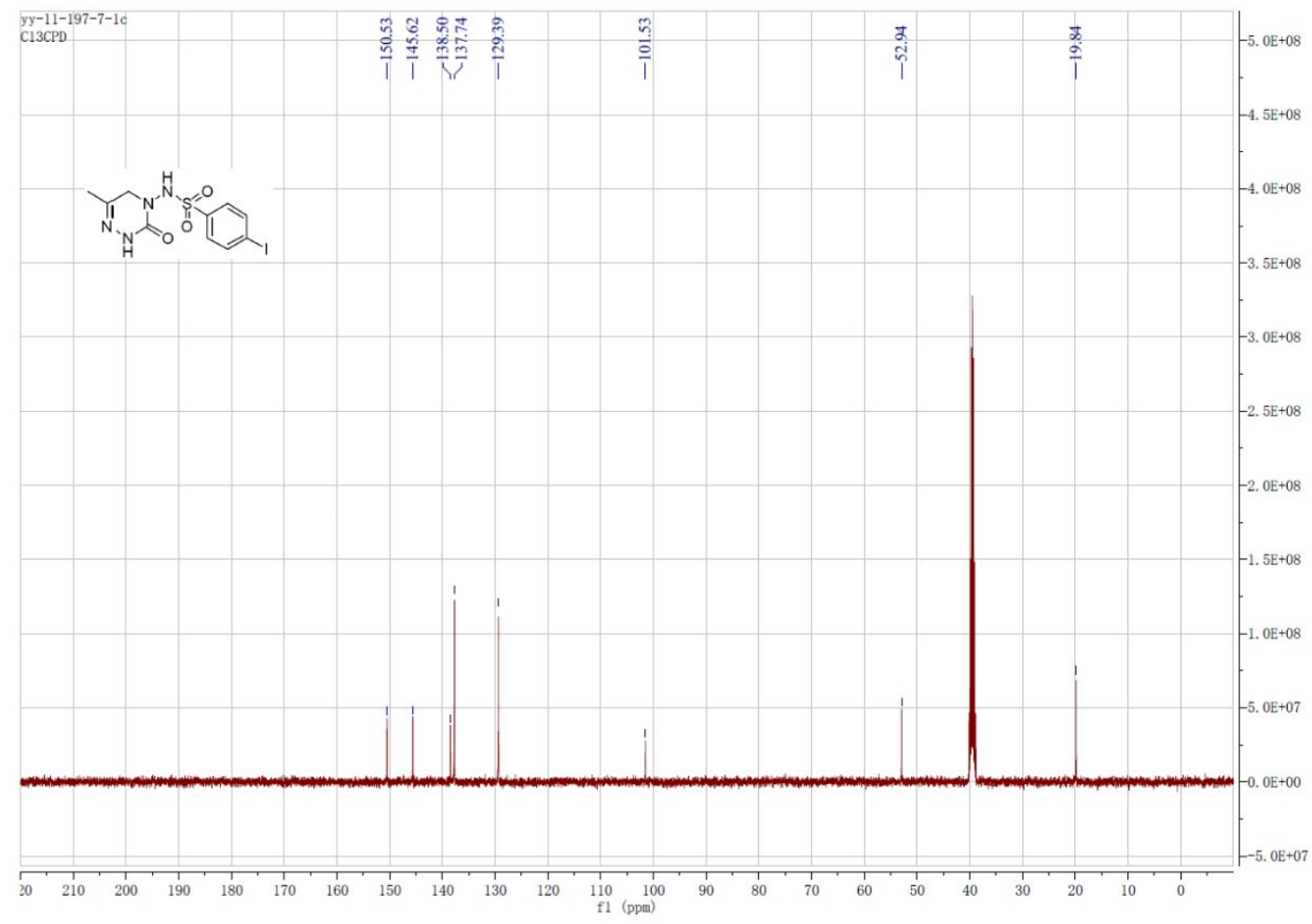


I-25 ${ }^{1} \mathrm{H}$ NMR (400 MHz, DMSO- $d_{6}$ )

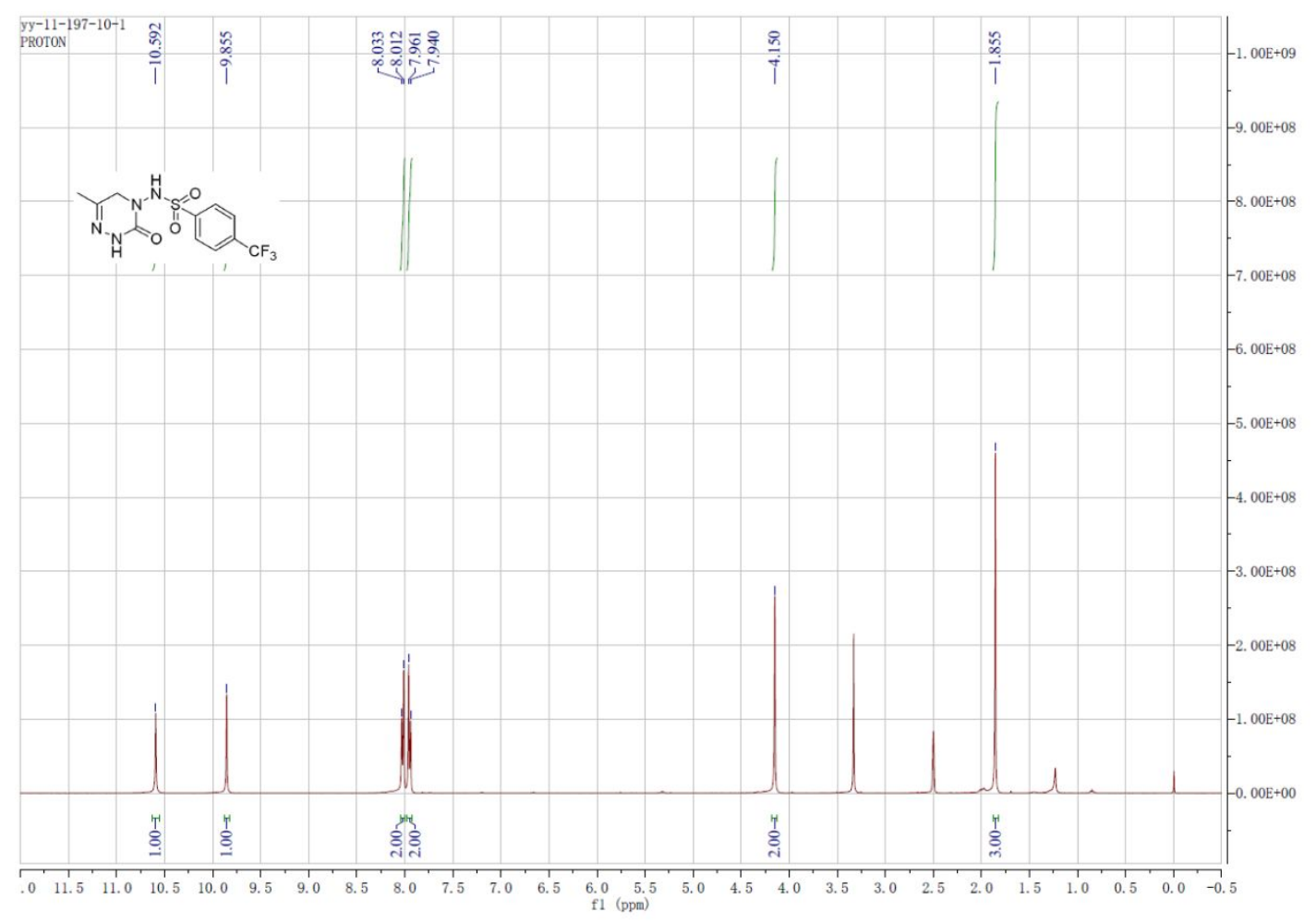

I-25 ${ }^{13} \mathrm{C}$ NMR (100 MHz, DMSO- $\left.d_{6}\right)$

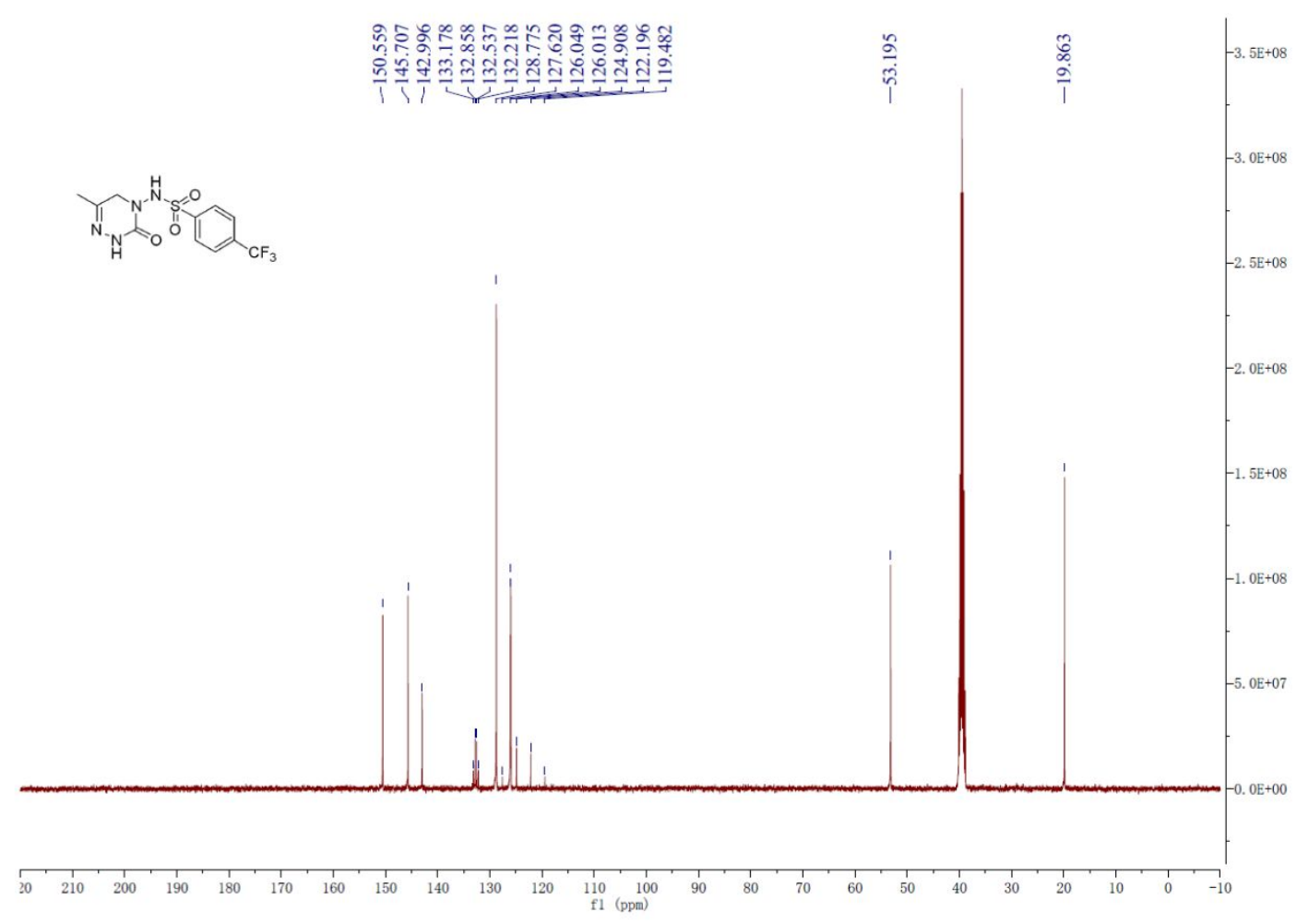


I-26 ${ }^{1} \mathrm{H}$ NMR (400 MHz, DMSO- $\left.d_{6}\right)$

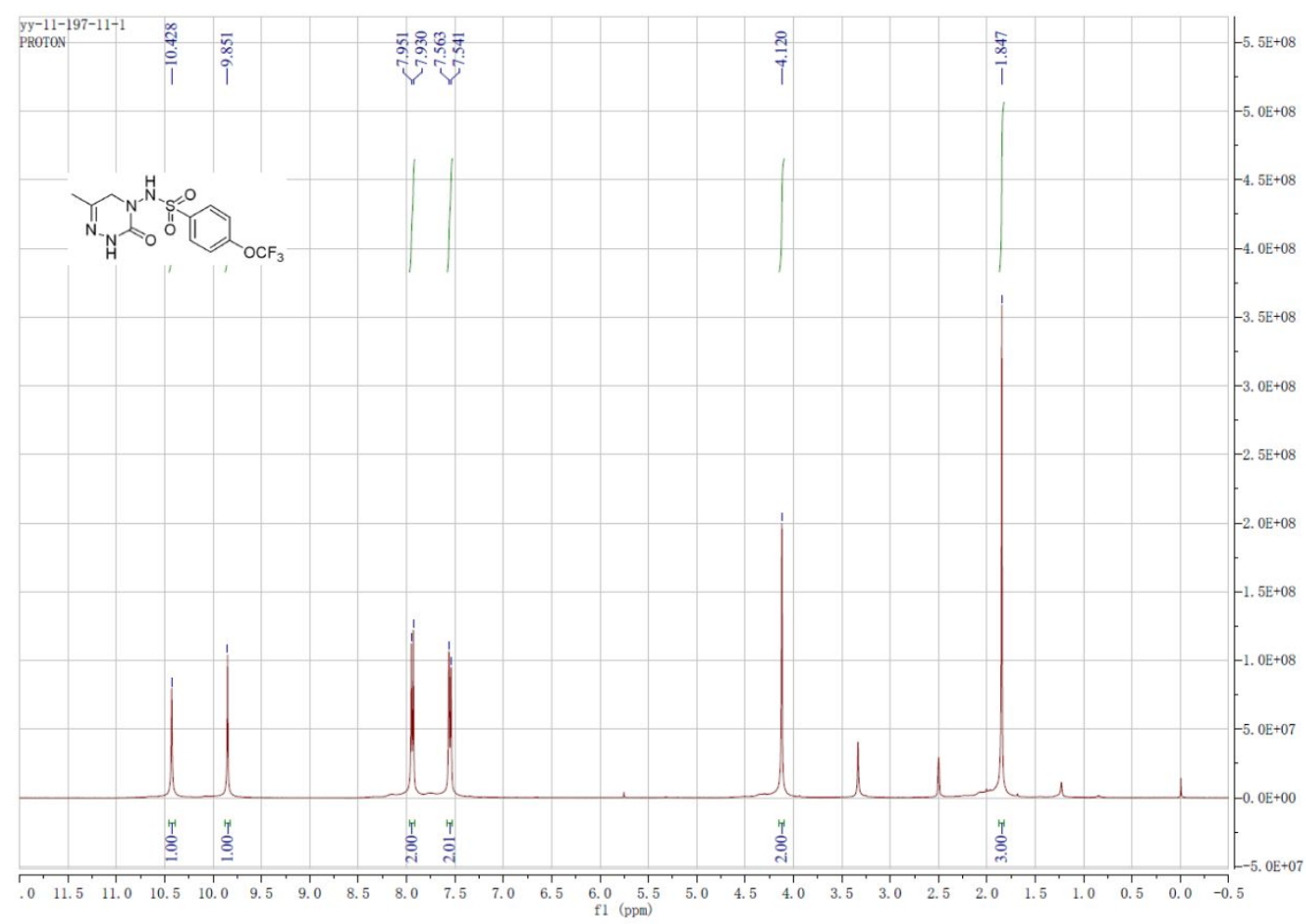

\section{I-26 ${ }^{13} \mathrm{C}$ NMR (100 MHz, DMSO- $\left.d_{6}\right)$}

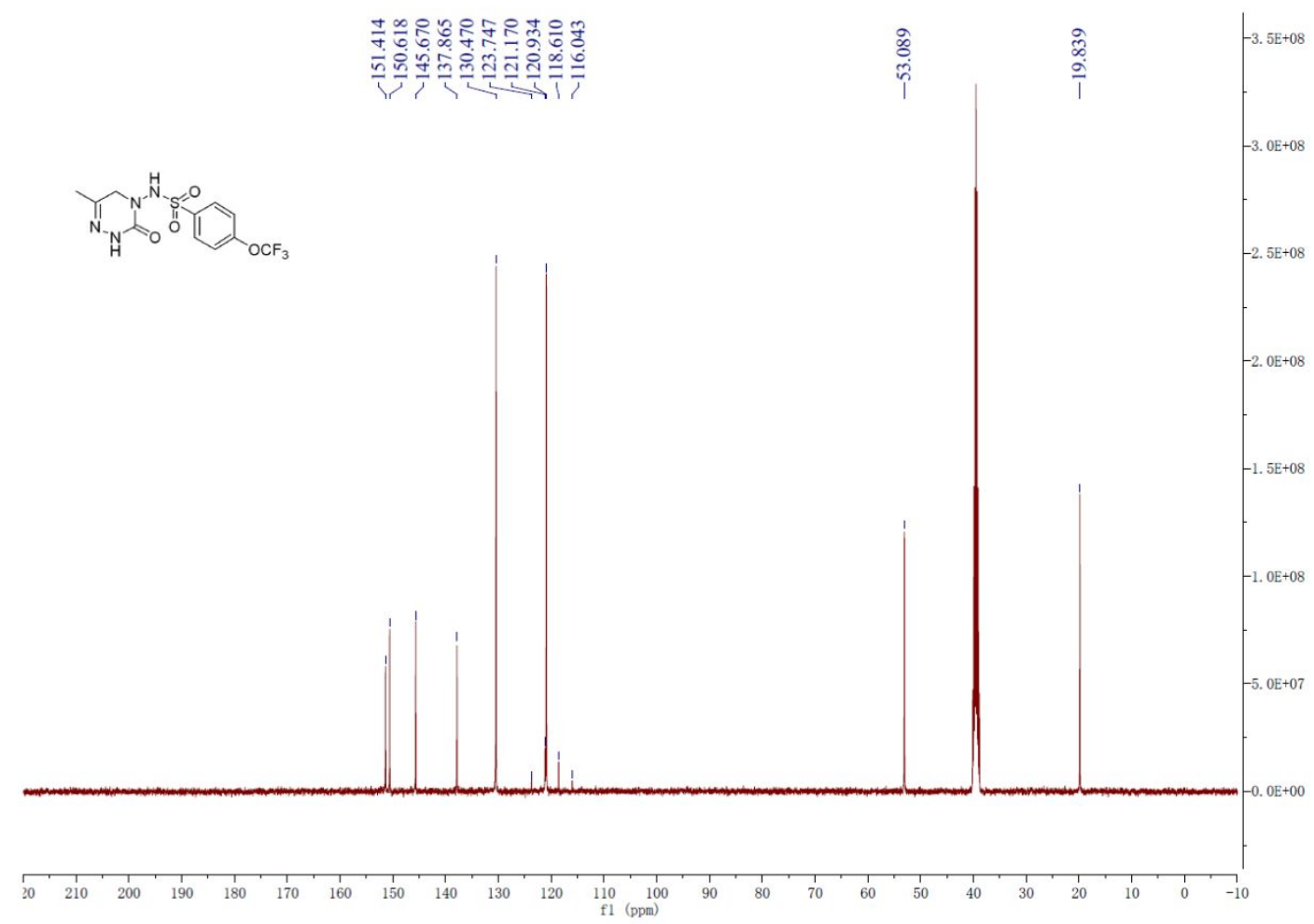


I-27 ${ }^{1} \mathrm{H}$ NMR (400 MHz, DMSO- $d_{6}$ )

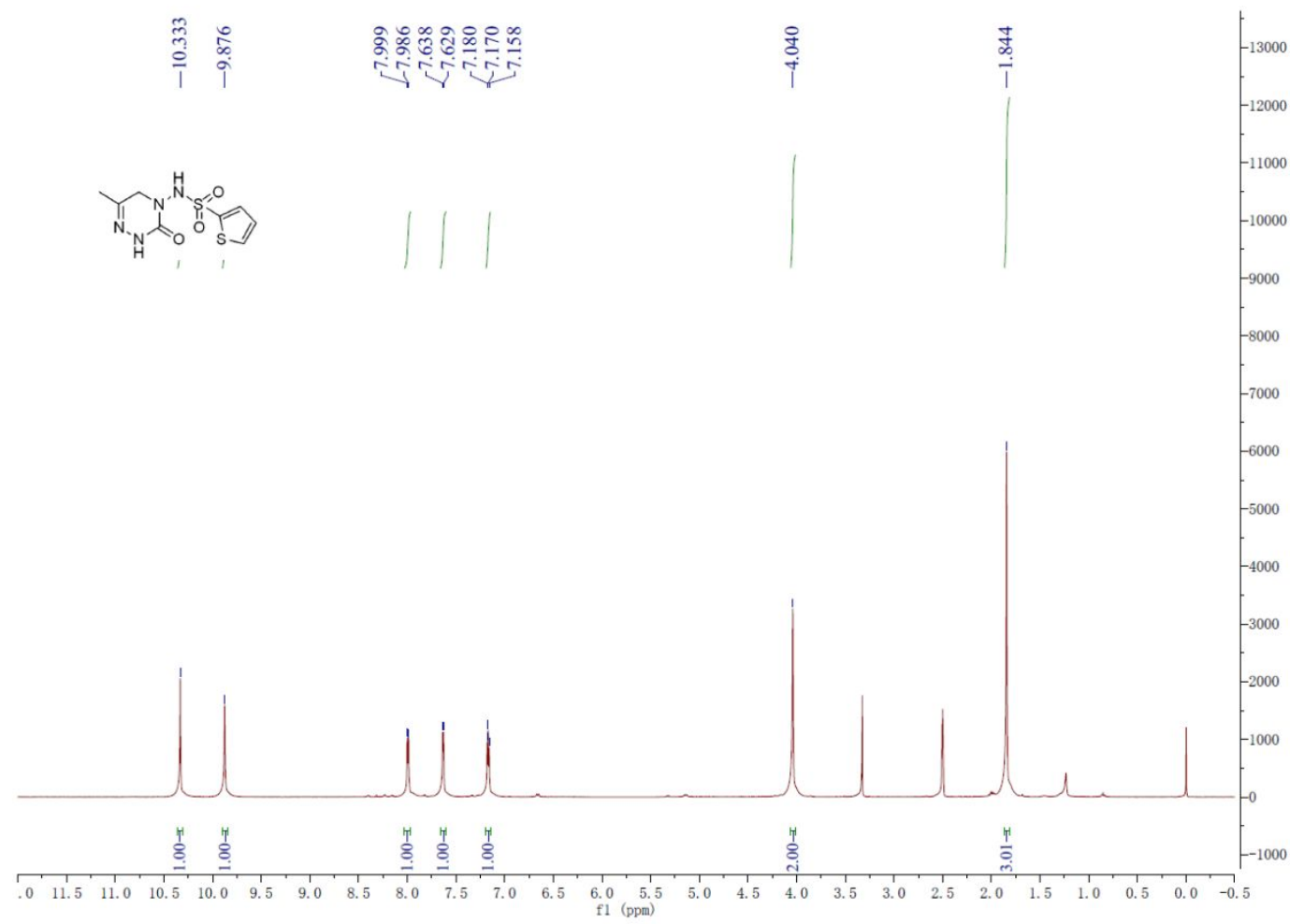

I-27 ${ }^{13} \mathrm{C}$ NMR (100 MHz, DMSO- $\left.d_{6}\right)$

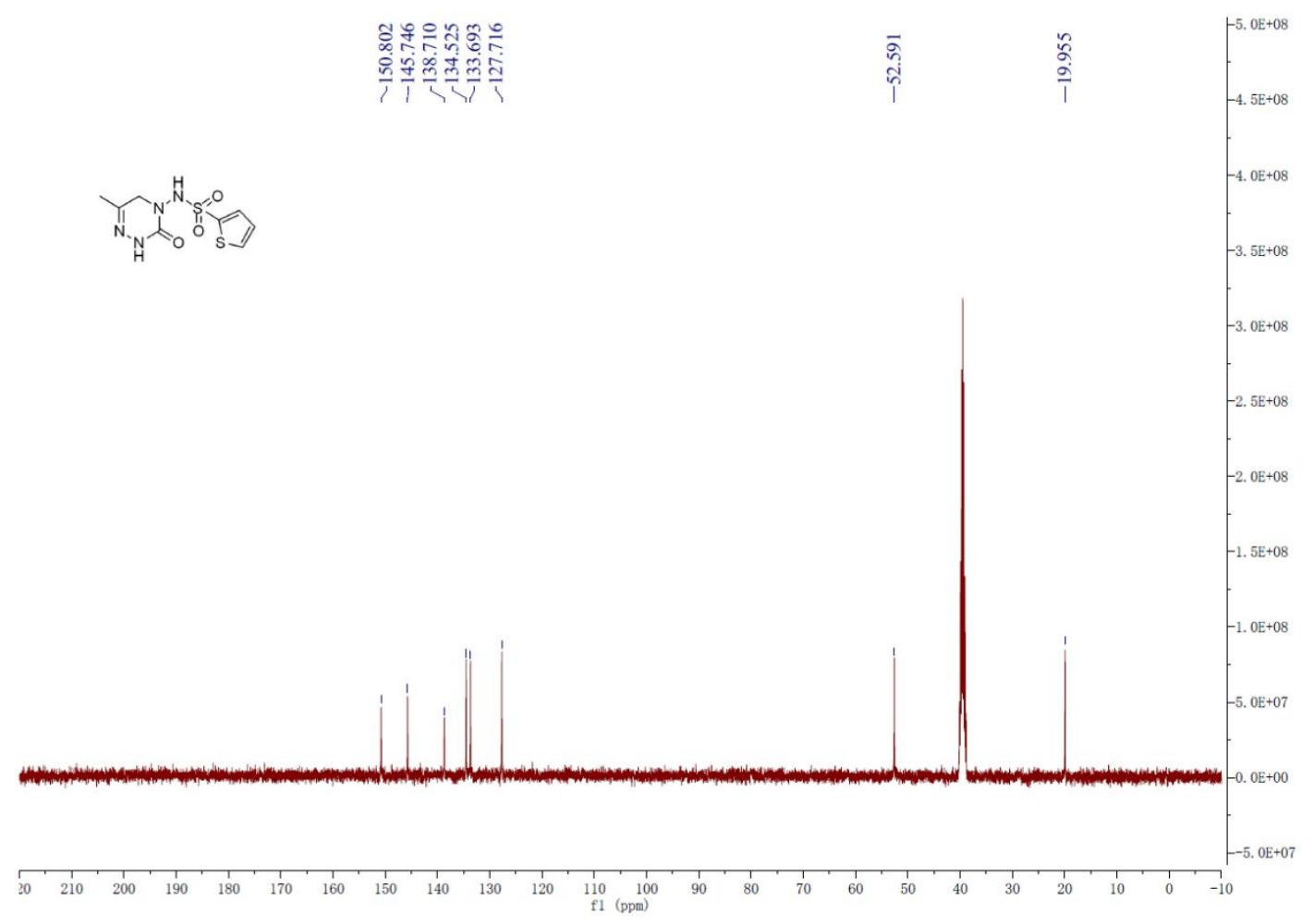


I-28 ${ }^{1} \mathrm{H}$ NMR (400 MHz, DMSO- $\left.d_{6}\right)$

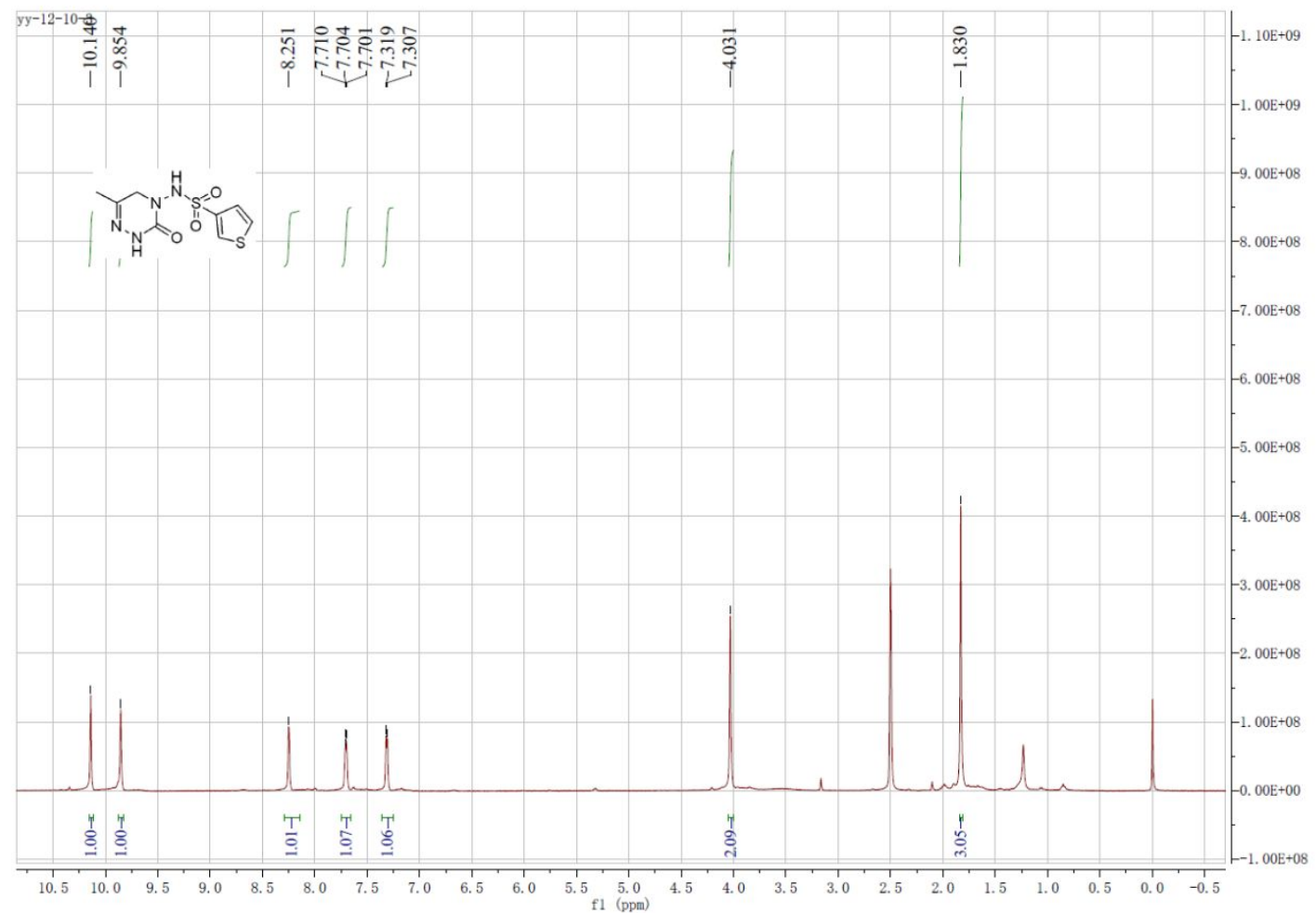

I-28 ${ }^{13} \mathrm{C}$ NMR (100 MHz, DMSO- $\left.d_{6}\right)$

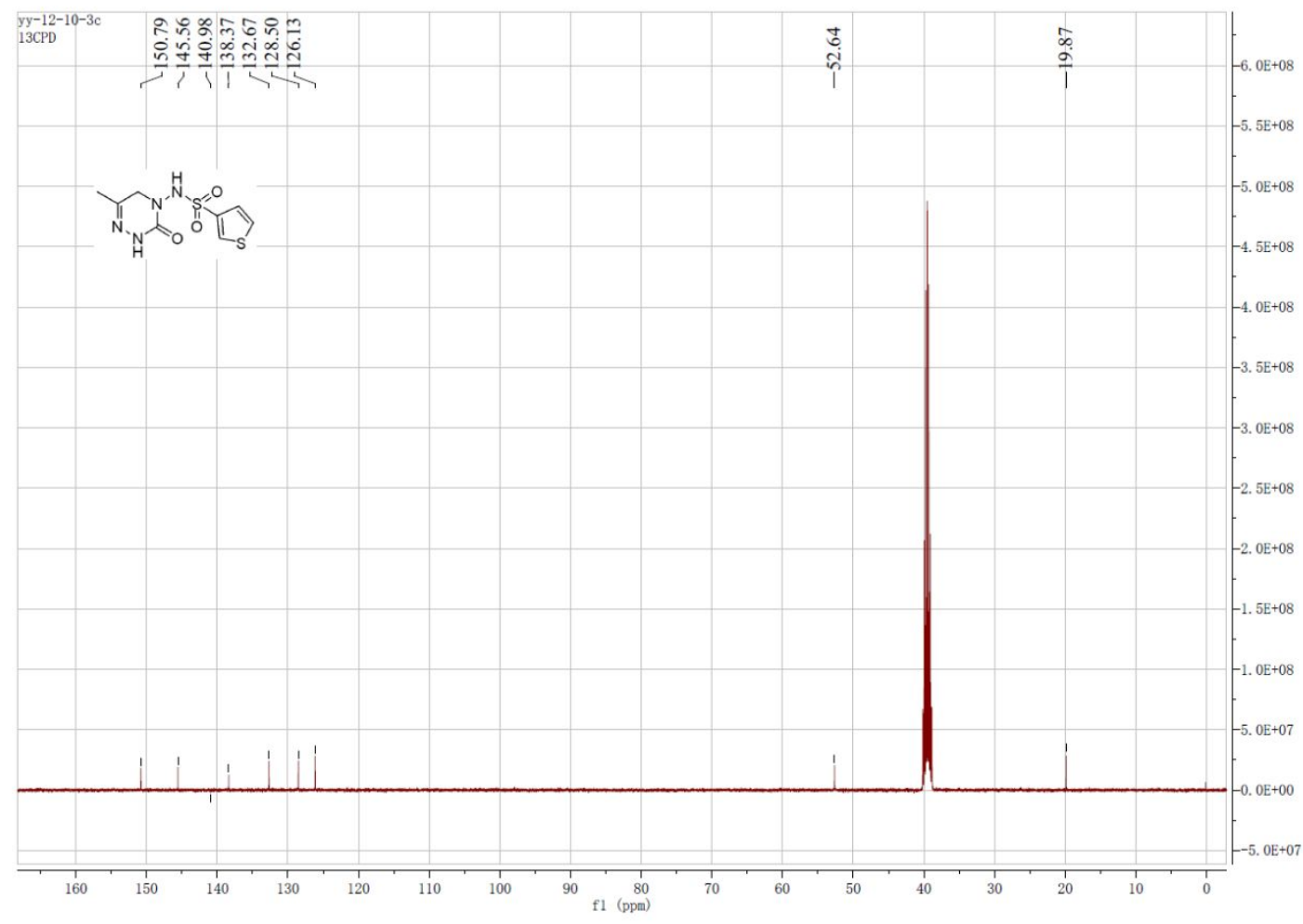


I-29 ${ }^{1} \mathrm{H}$ NMR $\left(400 \mathrm{MHz}, \mathrm{DMSO}-d_{6}\right)$

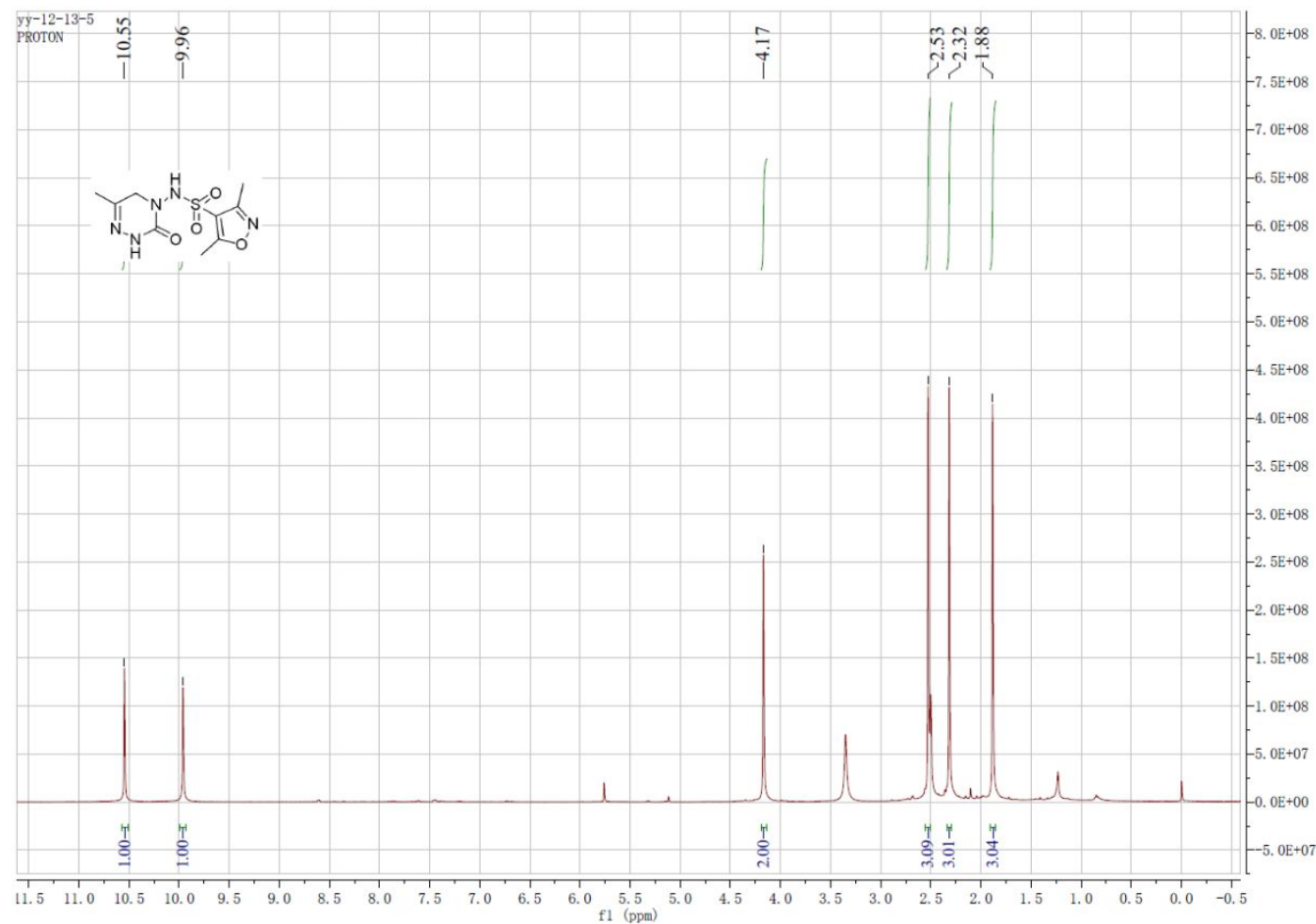

I-29 ${ }^{13} \mathrm{C}$ NMR (100 MHz, DMSO- $\left.d_{6}\right)$

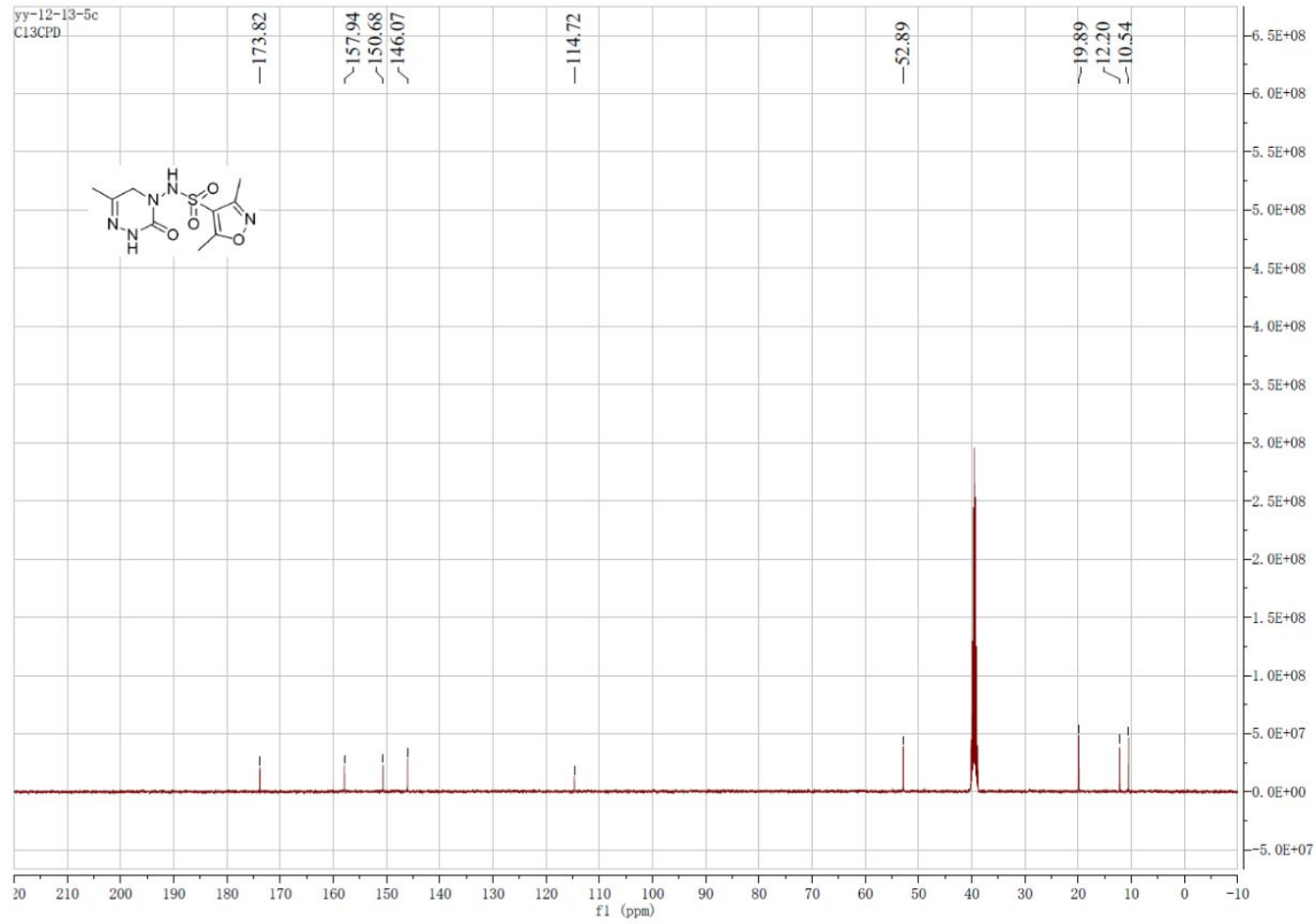




\section{I-30 ${ }^{1} \mathrm{H}$ NMR (400 MHz, DMSO- $\left.d_{6}\right)$}

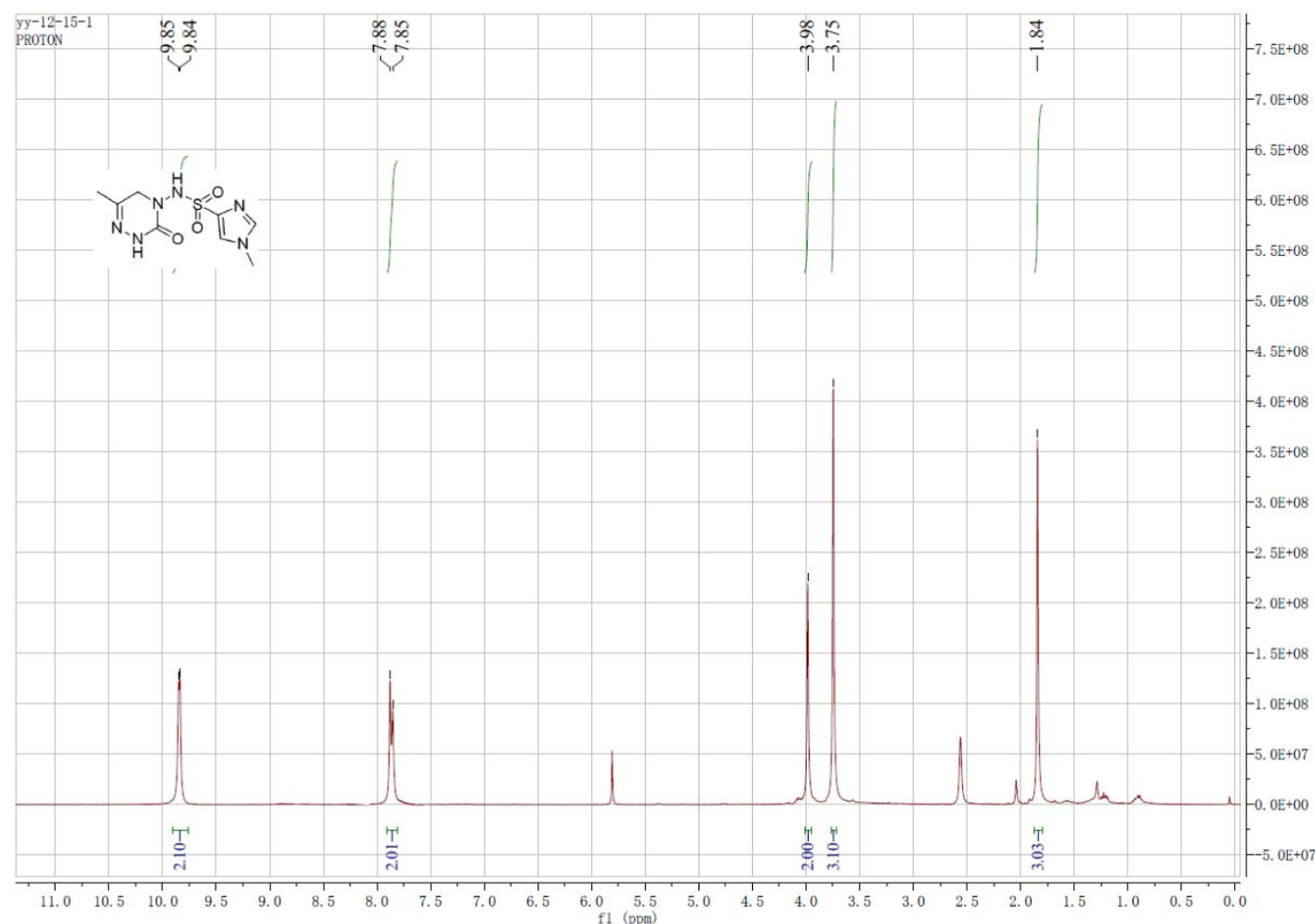

I-30 ${ }^{13} \mathrm{C}$ NMR (100 MHz, DMSO- $\left.d_{6}\right)$

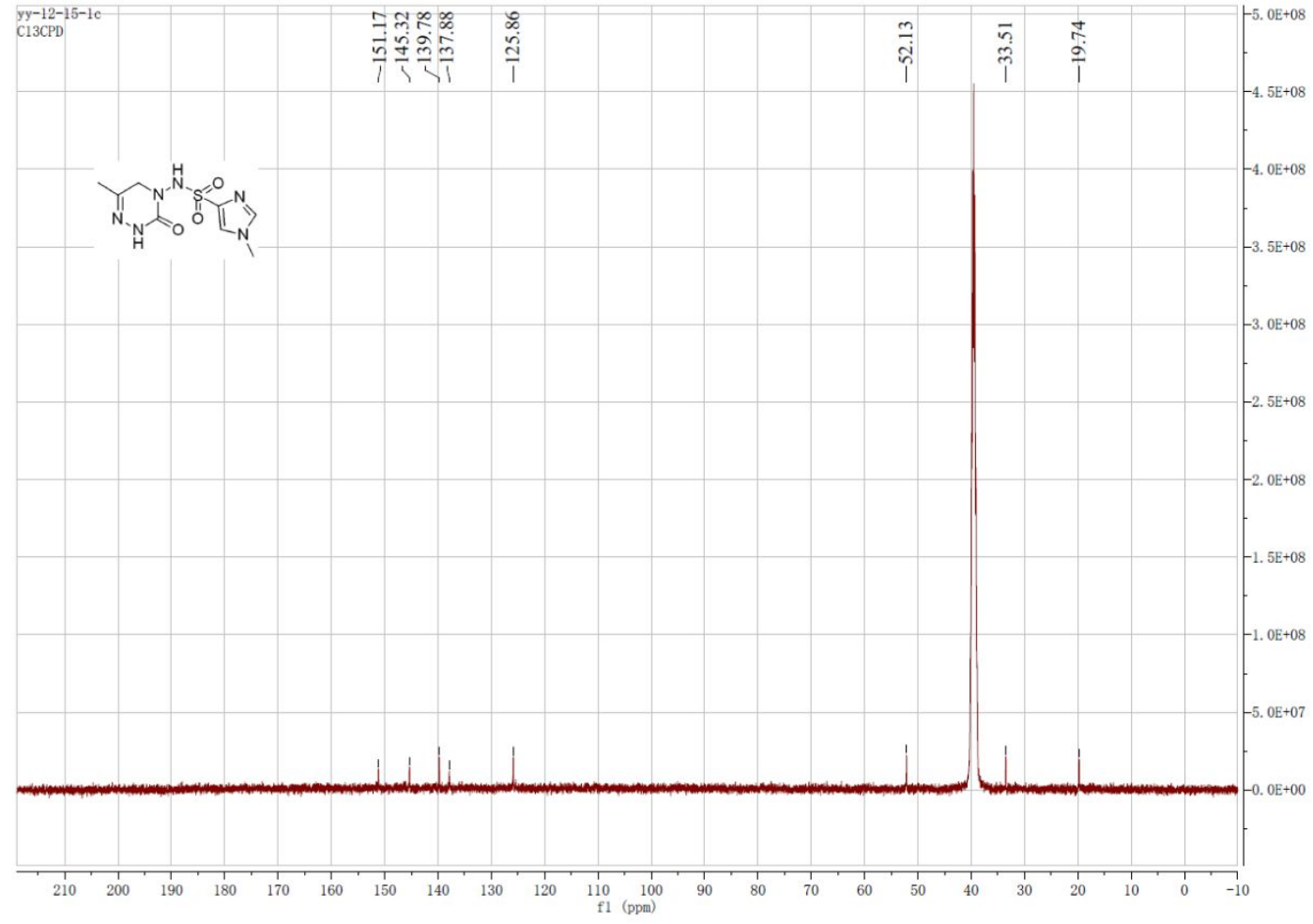


I-31 ${ }^{1} \mathrm{H}$ NMR (400 MHz, DMSO- $\left.d_{6}\right)$

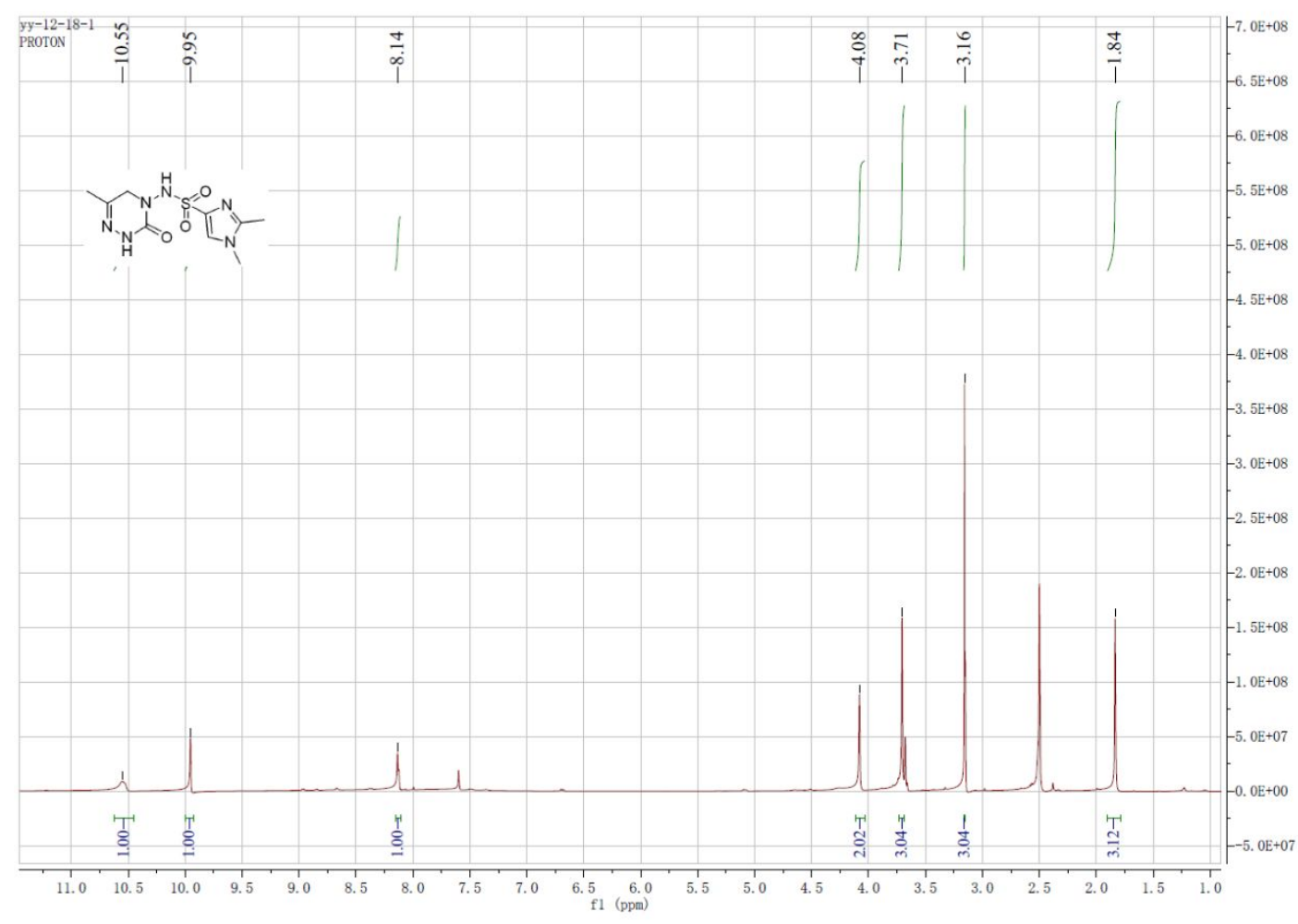

I-31 ${ }^{13} \mathrm{C}$ NMR (100 MHz, DMSO- $d_{6}$ )

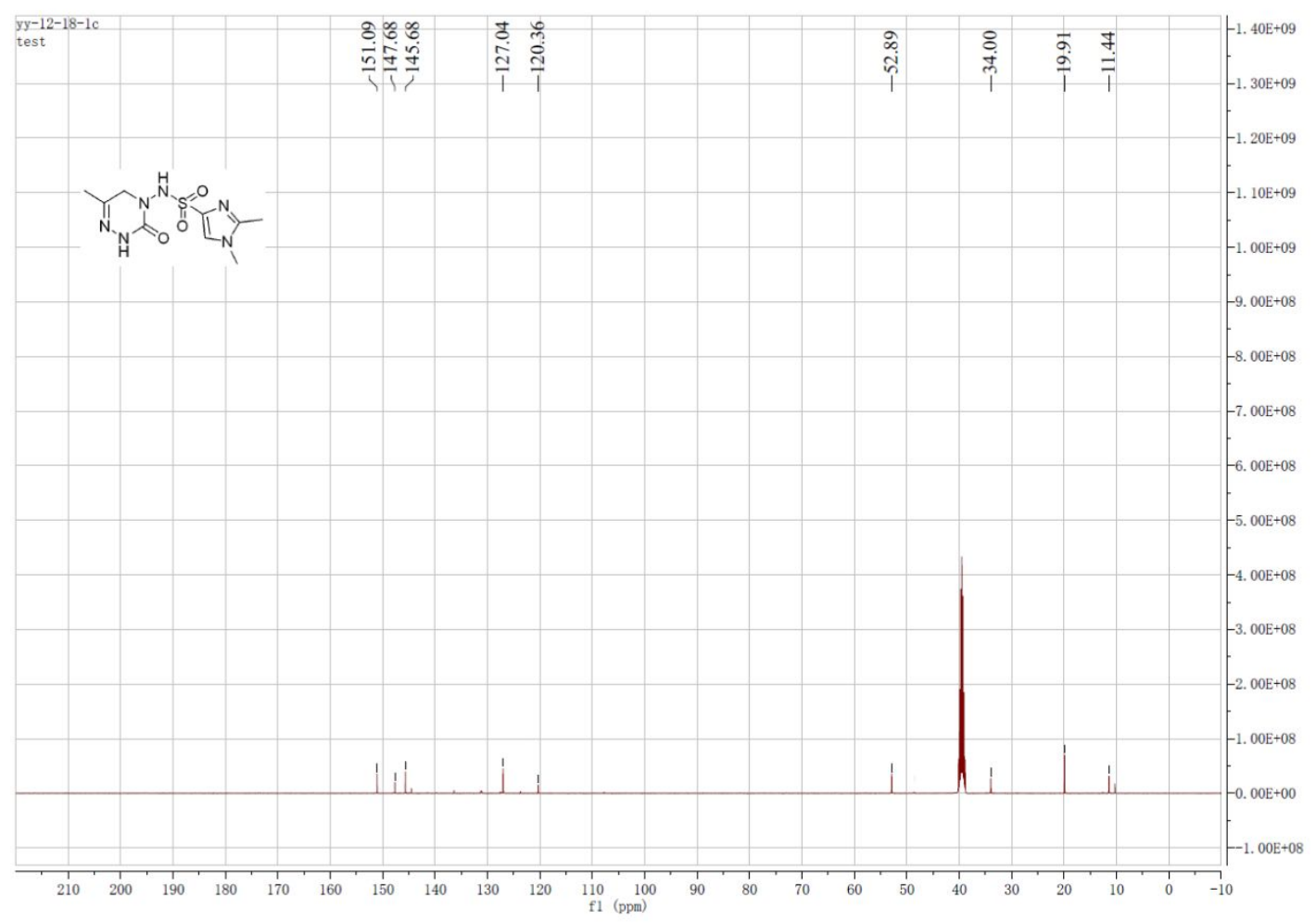


I-32 ${ }^{1} \mathrm{H}$ NMR (400 MHz, DMSO- $\left.d_{6}\right)$

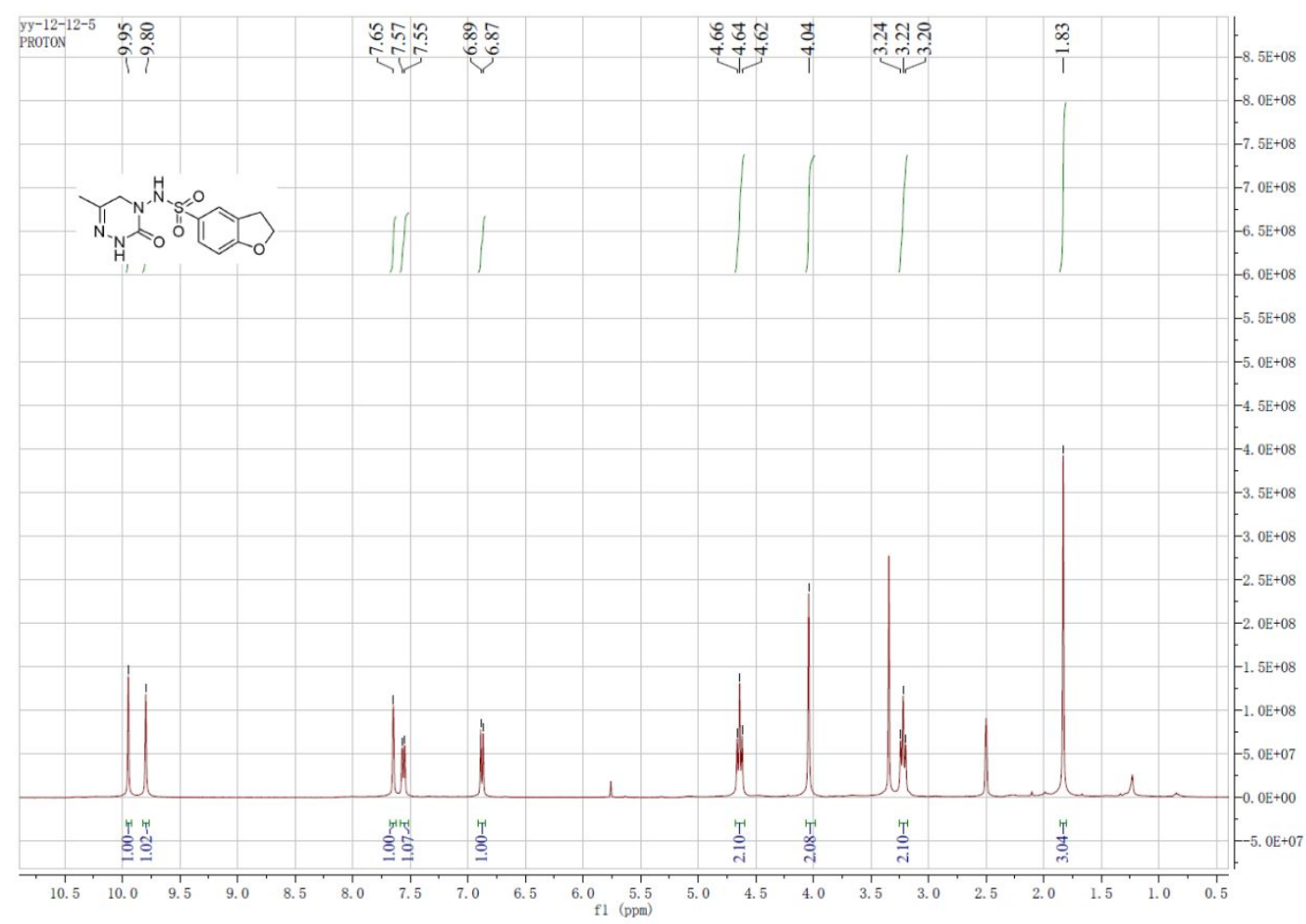

I-32 ${ }^{13} \mathrm{C}$ NMR (100 MHz, DMSO- $d_{6}$ )

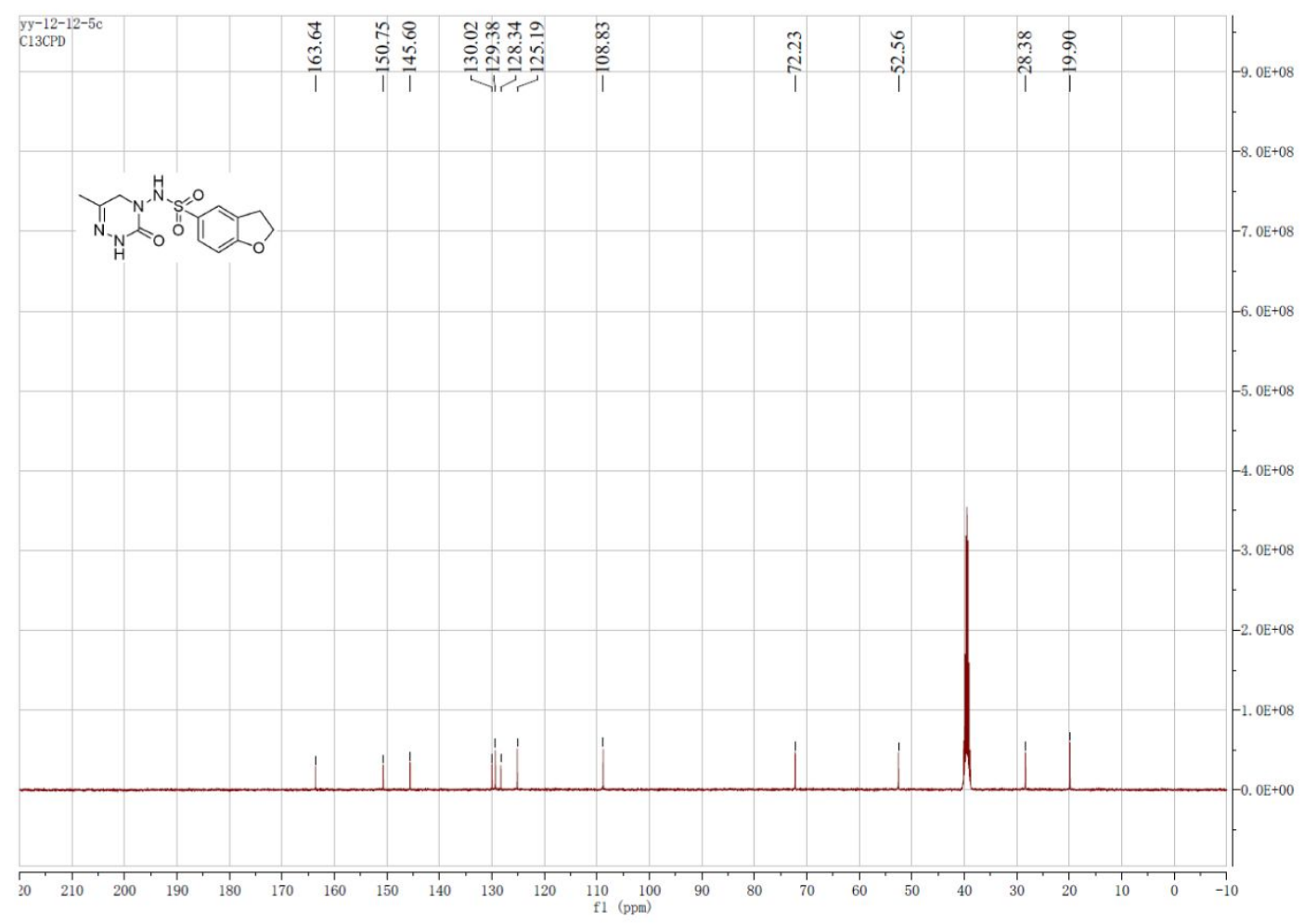


I-33 ${ }^{1} \mathrm{H}$ NMR (400 MHz, DMSO- $\left.d_{6}\right)$

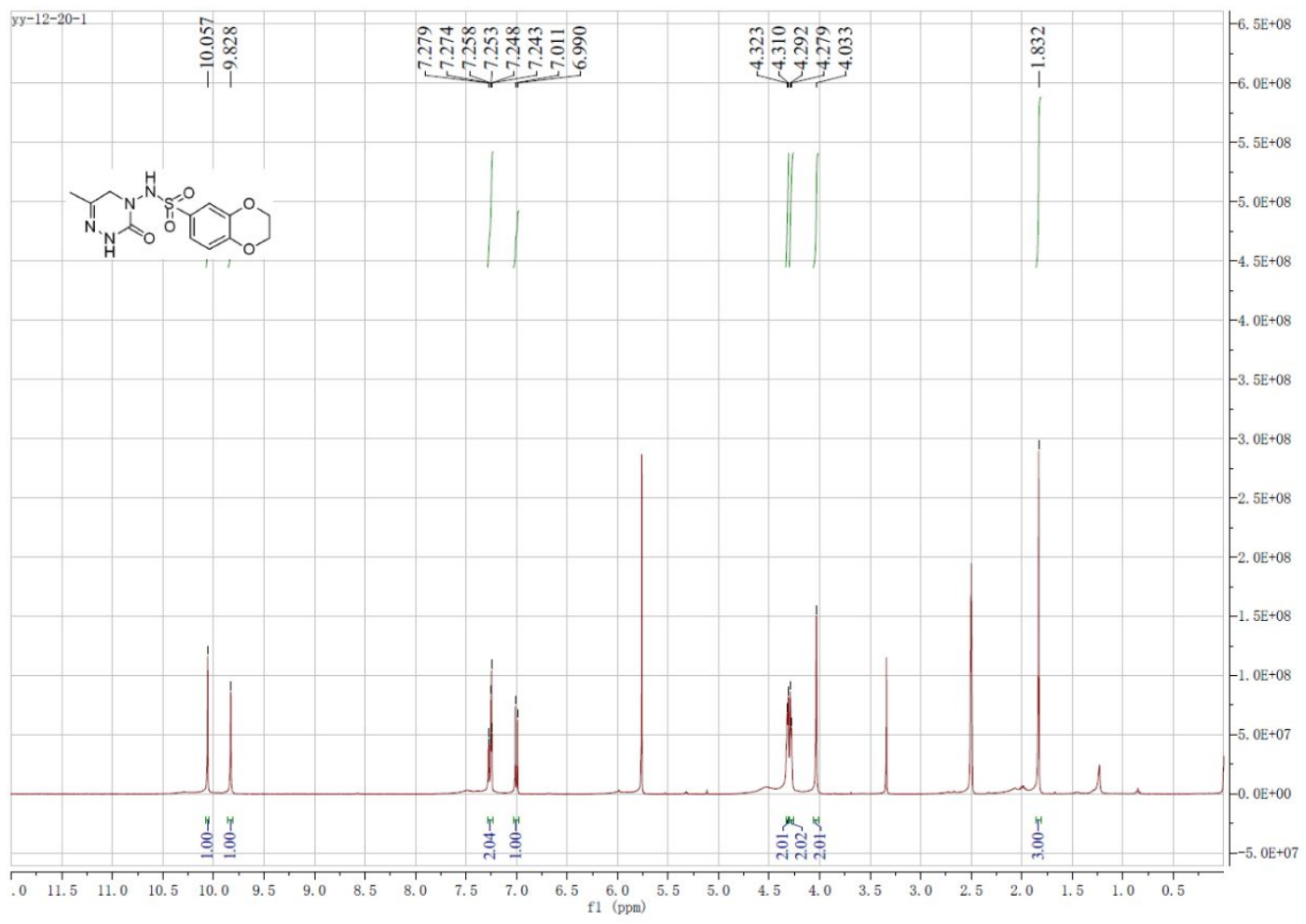

I-33 ${ }^{13} \mathrm{C}$ NMR (100 MHz, DMSO- $\left.d_{6}\right)$

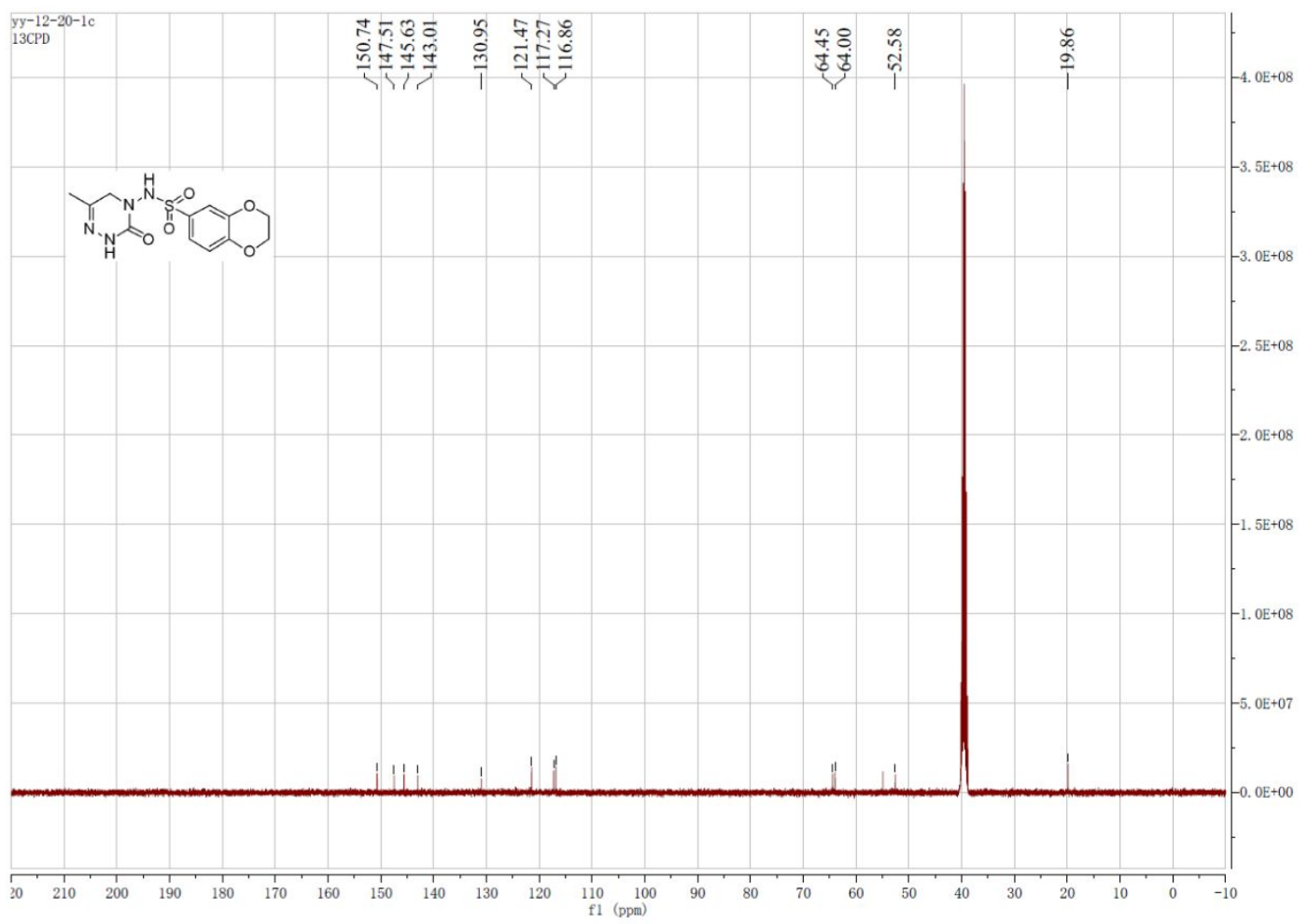

S49 
II-1 ${ }^{1} \mathrm{H}$ NMR (300 MHz, DMSO- $d_{6}$ )

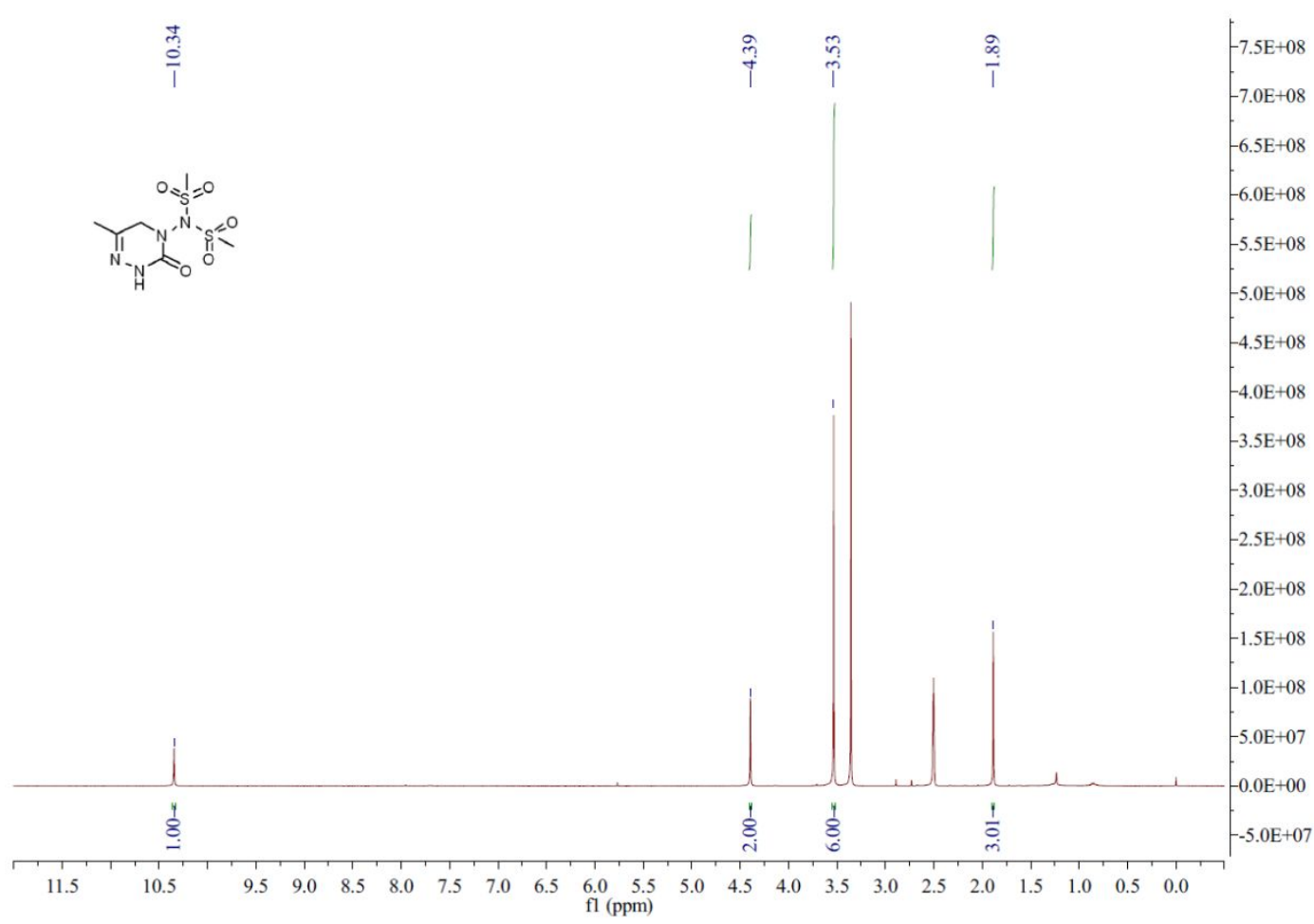

II-1 ${ }^{13} \mathrm{C}$ NMR (100 MHz, DMSO- $\left.d_{6}\right)$

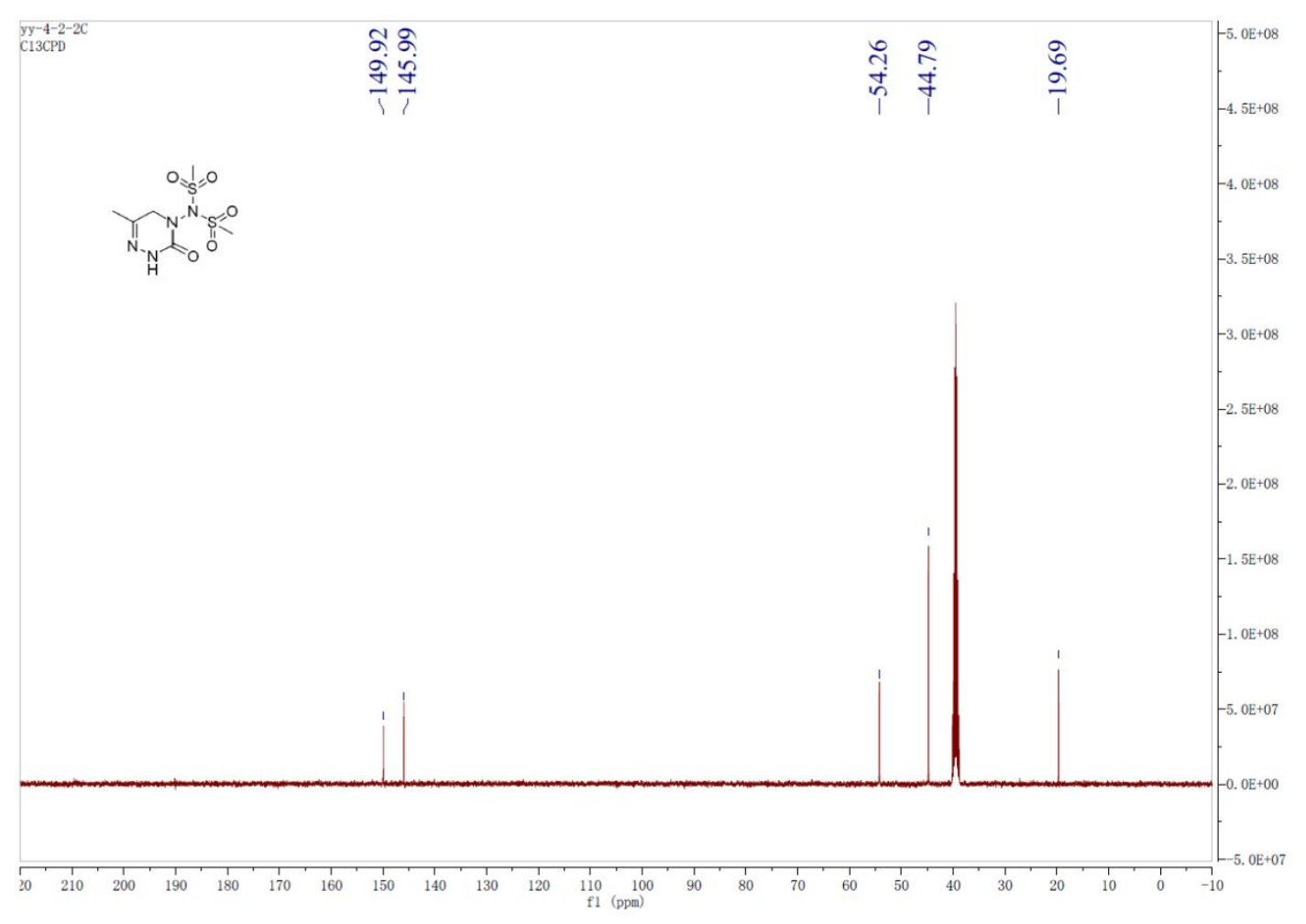


II-2 ${ }^{1} \mathrm{H}$ NMR (400 MHz, DMSO- $d_{6}$ )

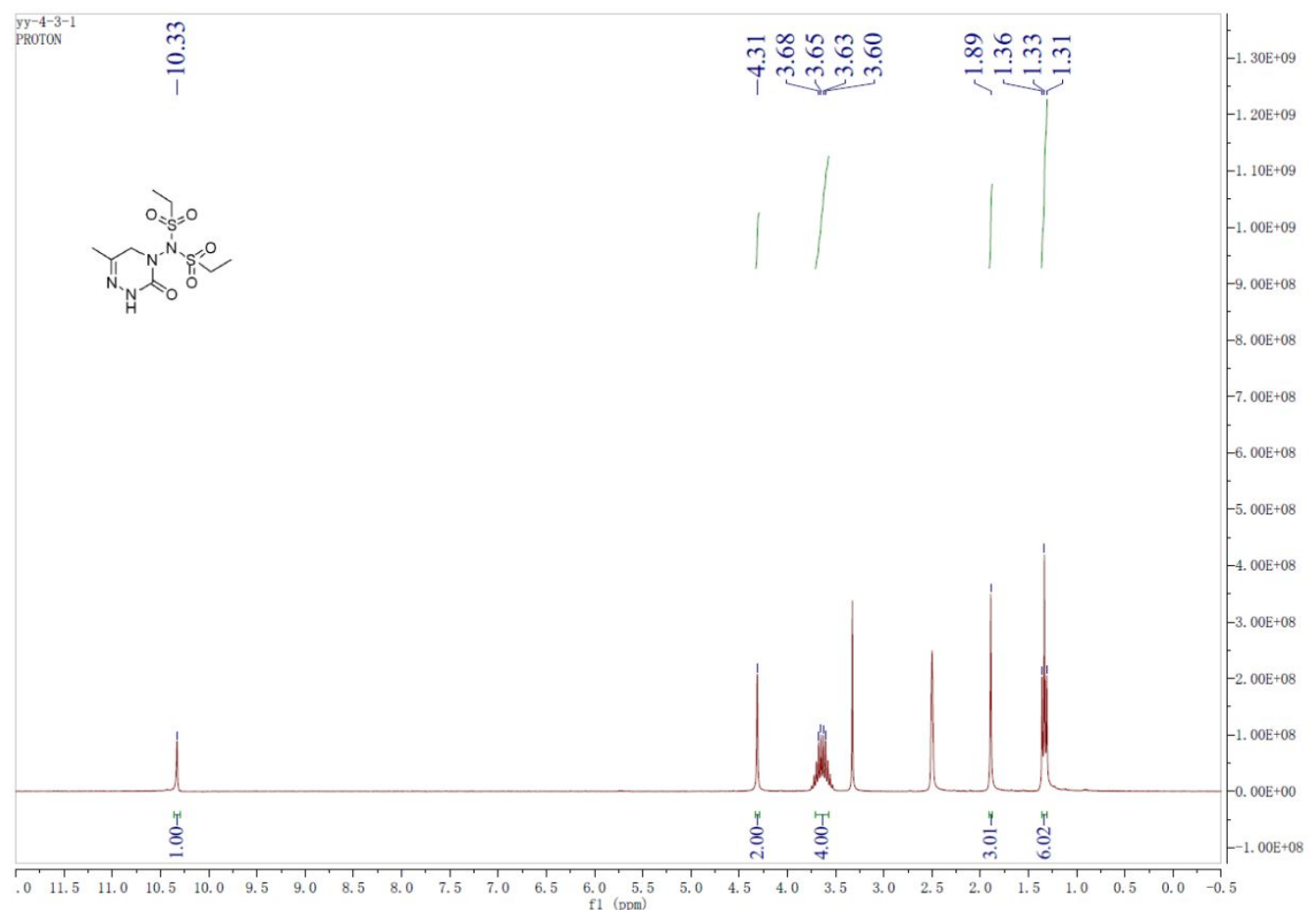

II-2 ${ }^{13} \mathrm{C}$ NMR $\left(100 \mathrm{MHz}\right.$, DMSO- $\left.d_{6}\right)$

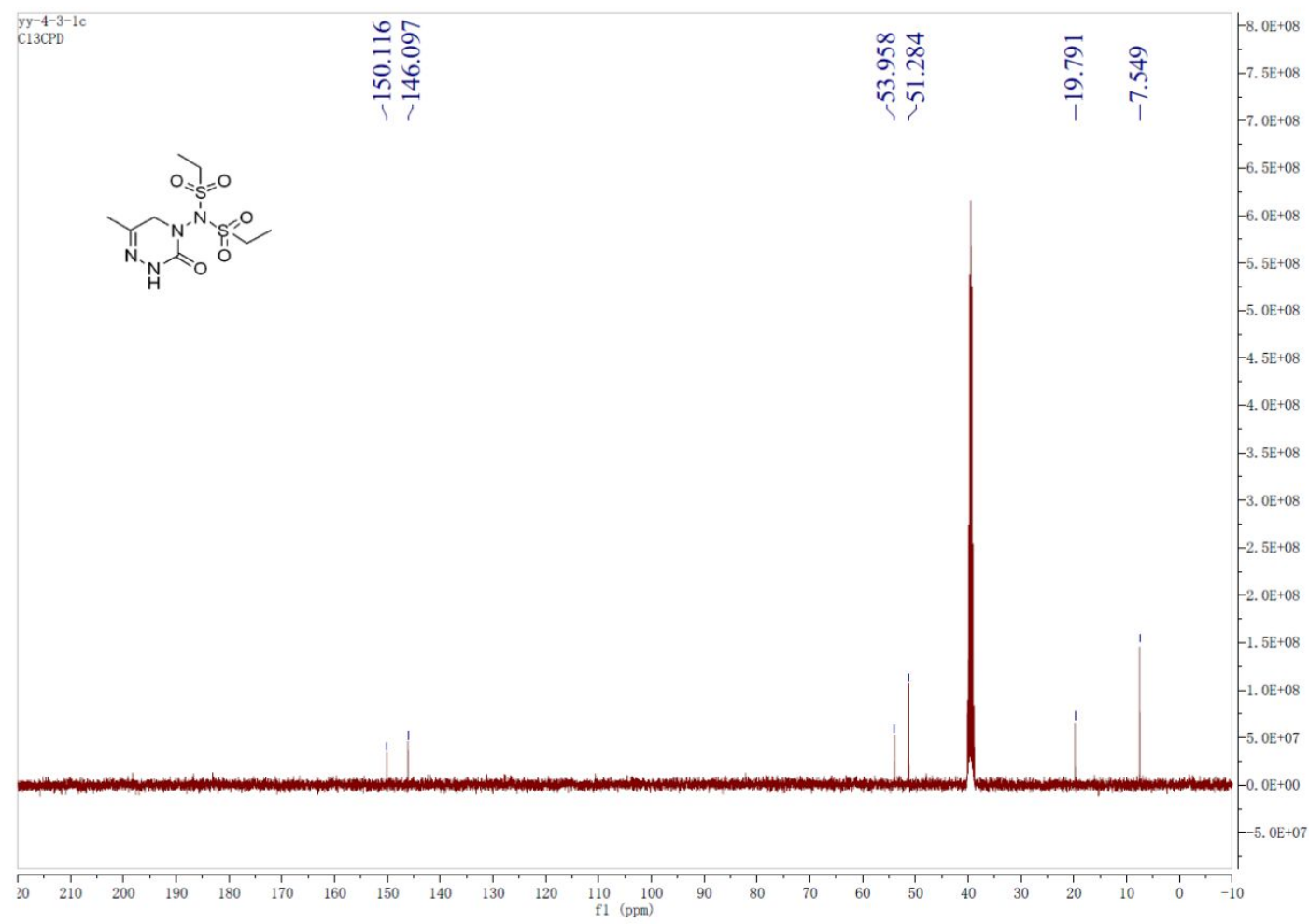


II-3 ${ }^{1} \mathrm{H}$ NMR (300 MHz, DMSO- $\left.d_{6}\right)$

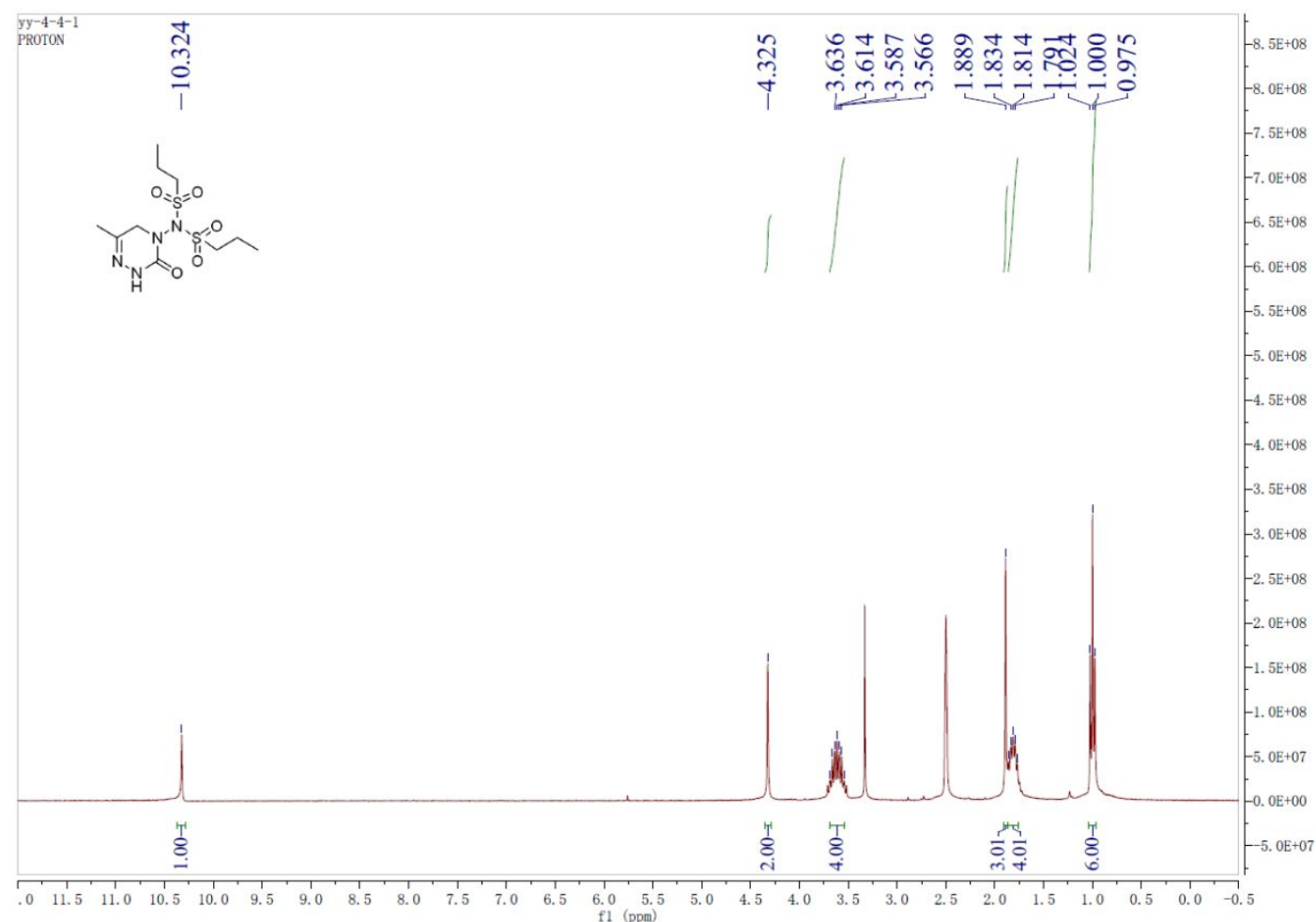

II-3 ${ }^{13} \mathrm{C}$ NMR (100 MHz, DMSO- $\left.d_{6}\right)$

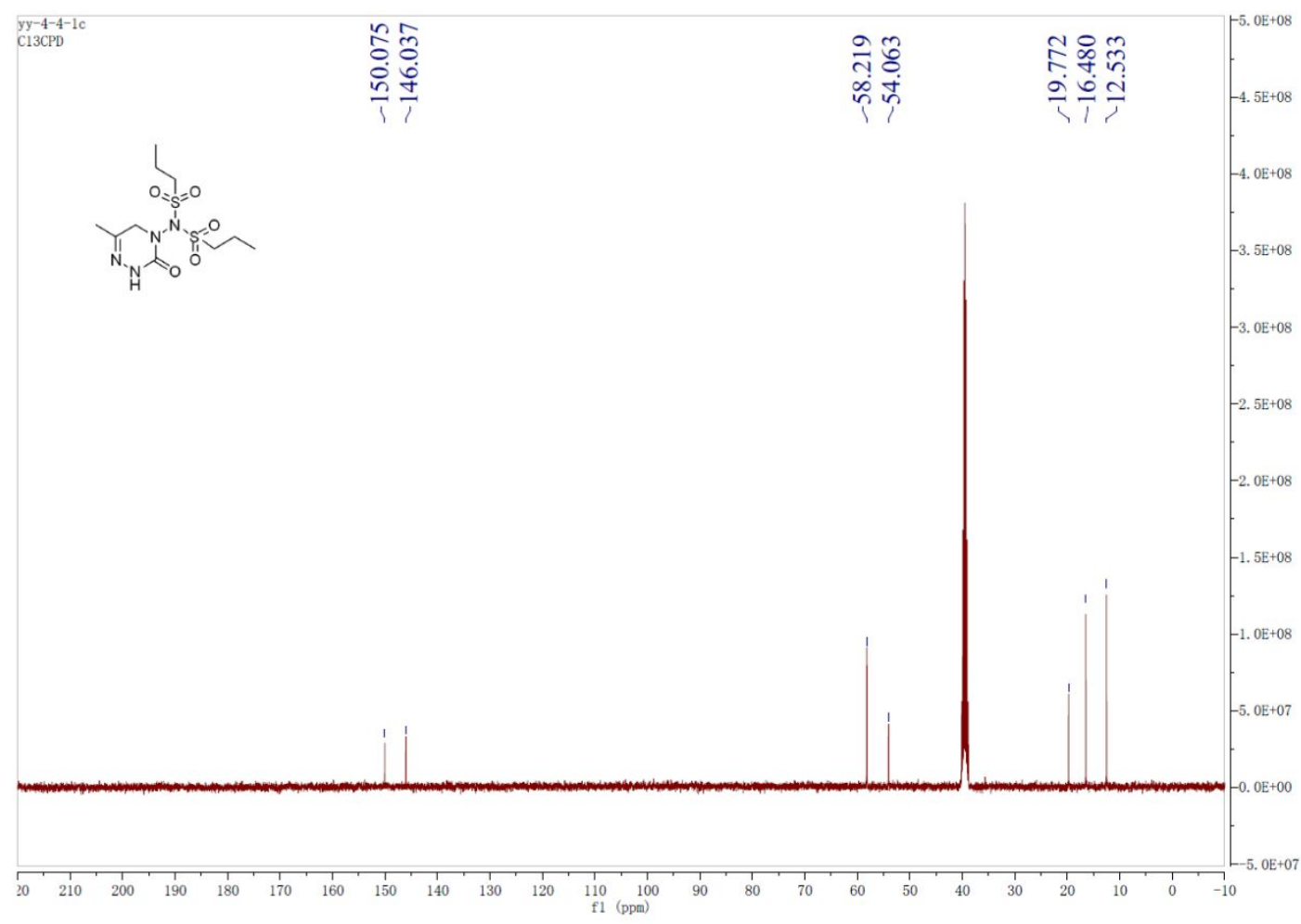


II-4 ${ }^{1} \mathrm{H}$ NMR $\left(300 \mathrm{MHz}, \mathrm{DMSO}-d_{6}\right)$

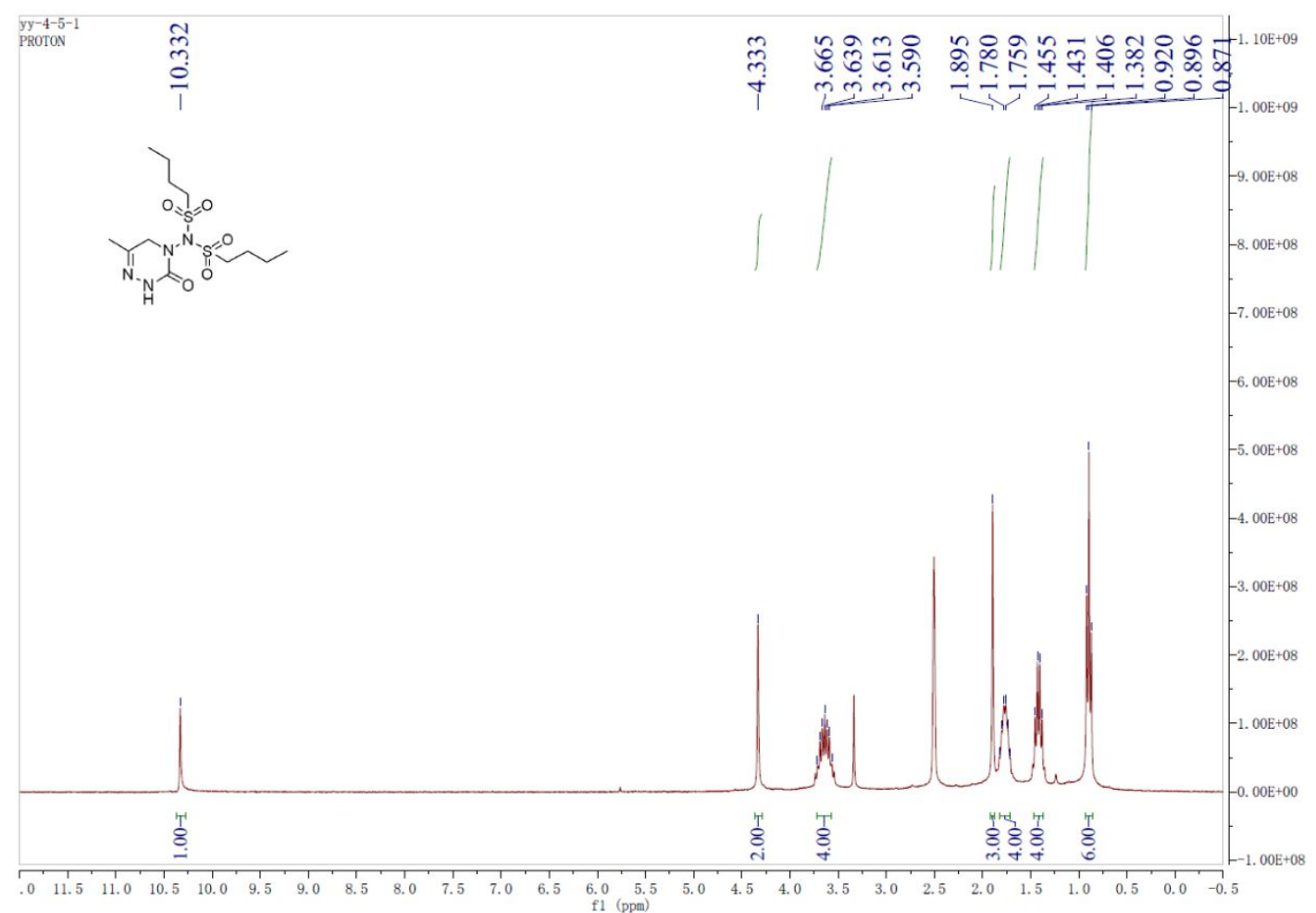

II-4 ${ }^{13} \mathrm{C}$ NMR $\left(100 \mathrm{MHz}\right.$, DMSO- $\left.d_{6}\right)$

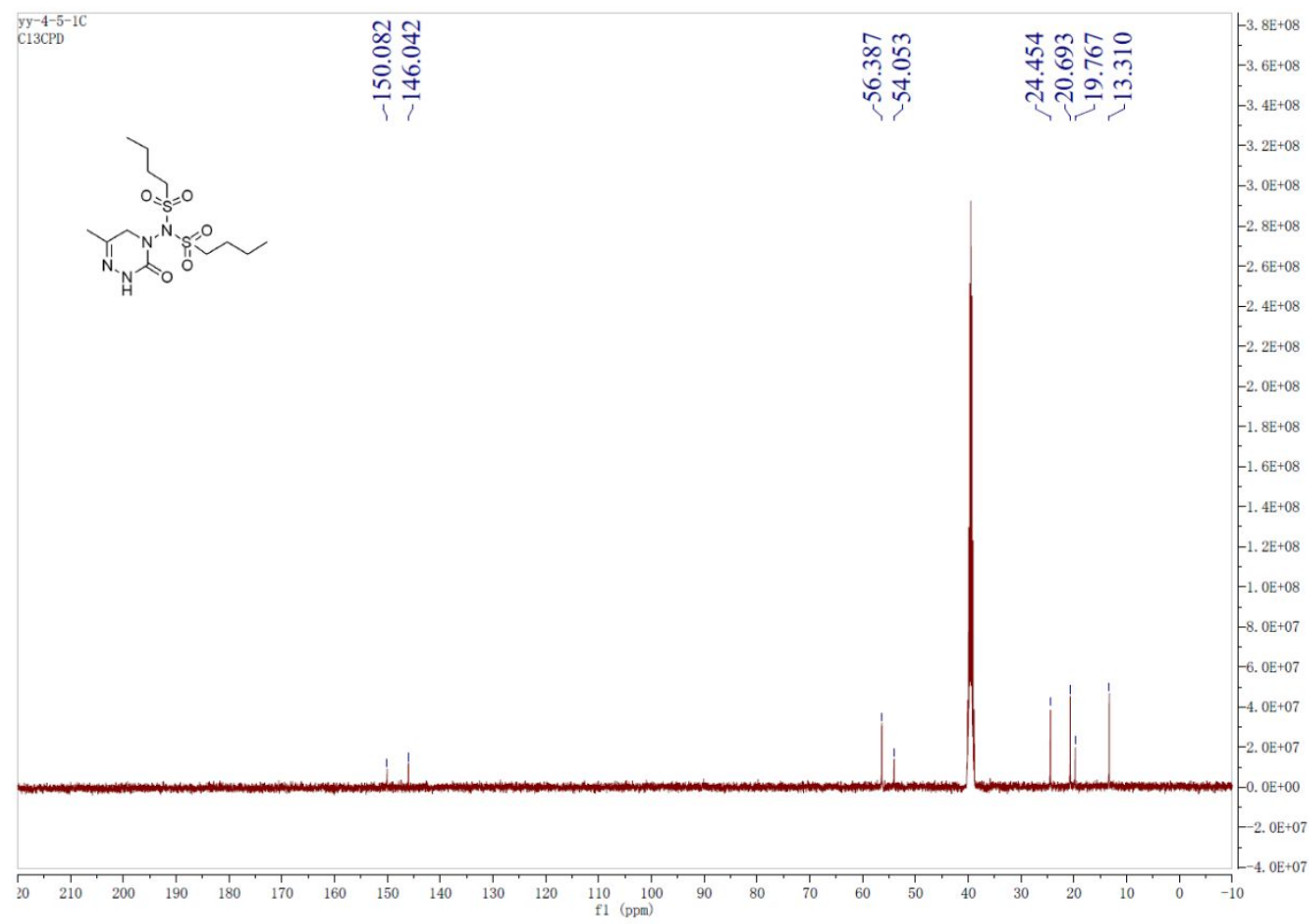


II-5 ${ }^{1} \mathrm{H}$ NMR (400 MHz, DMSO- $d_{6}$ )

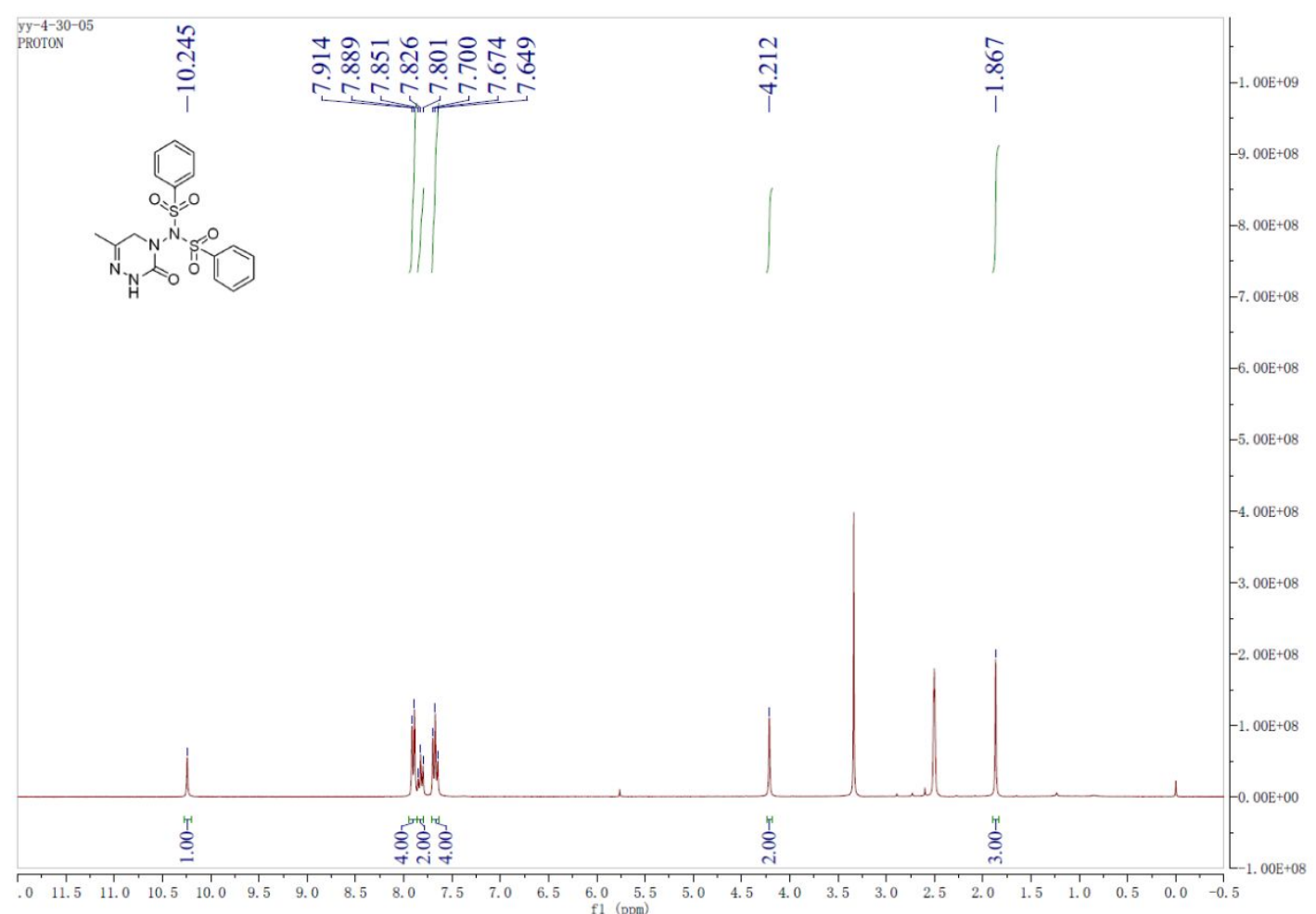

II-5 ${ }^{13} \mathrm{C}$ NMR (100 MHz, DMSO- $\left.d_{6}\right)$

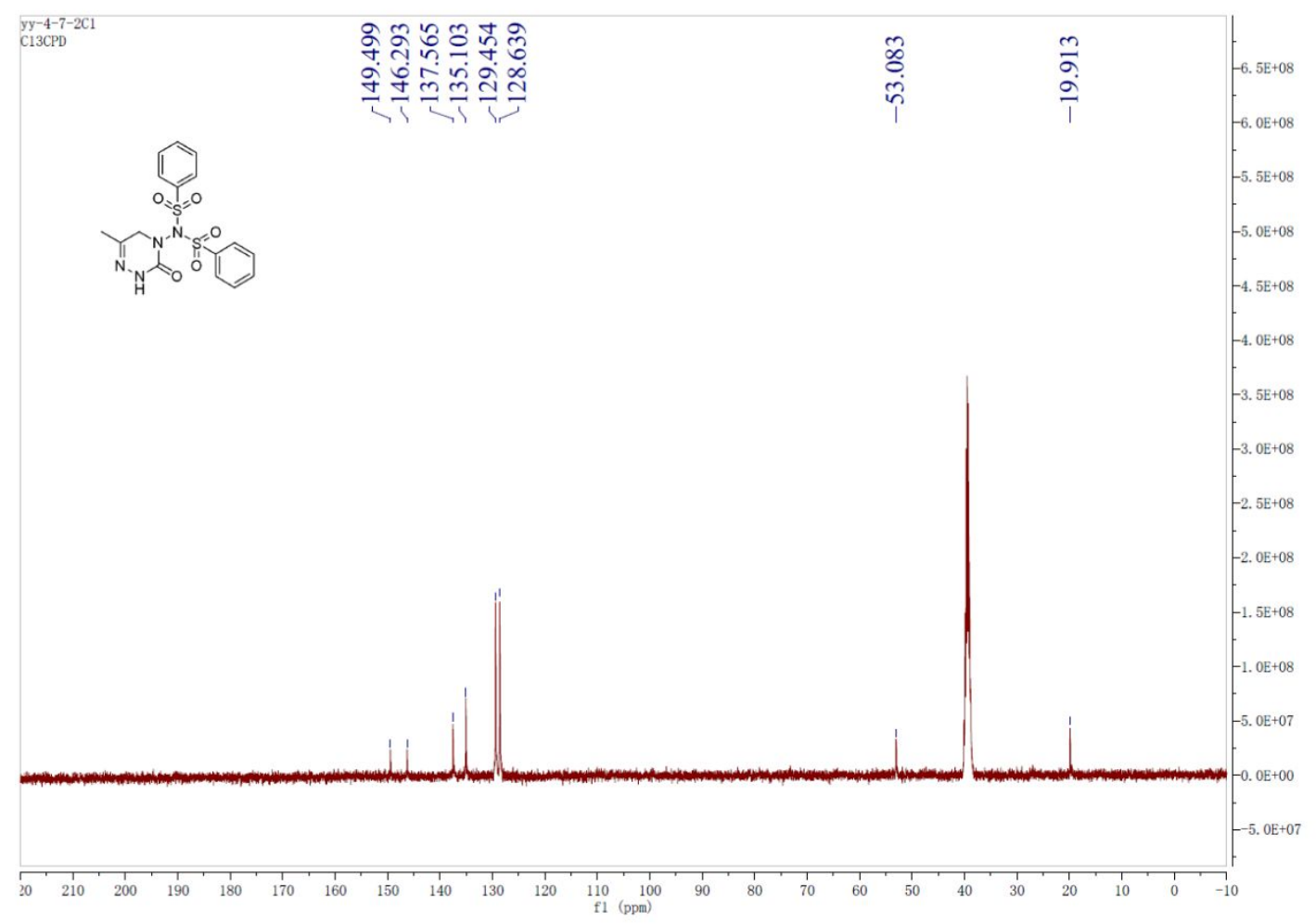


II-6 ${ }^{1} \mathrm{H}$ NMR (300 MHz, DMSO- $\left.d_{6}\right)$

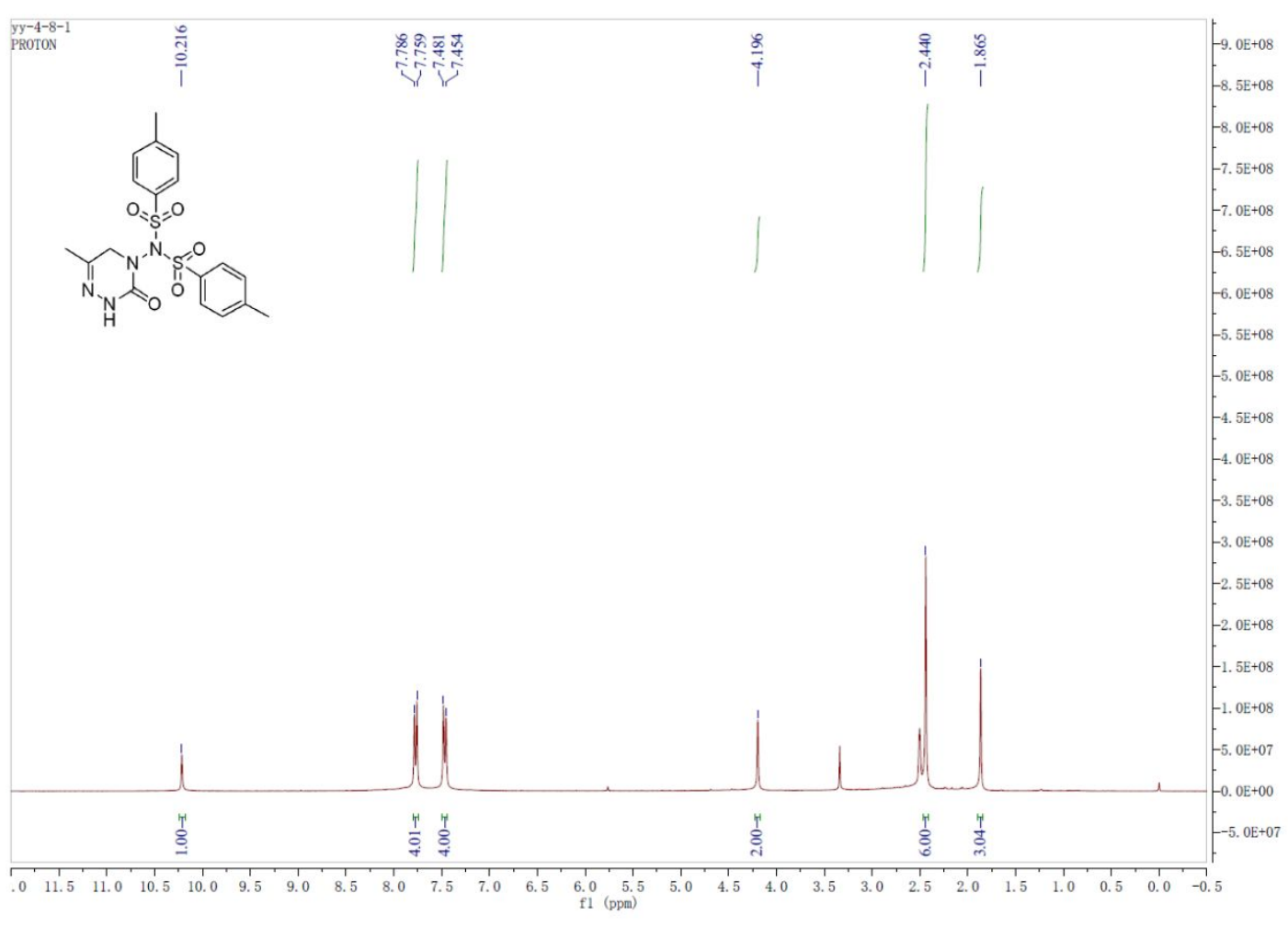

II-6 ${ }^{13} \mathrm{C}$ NMR (100 MHz, DMSO- $\left.d_{6}\right)$

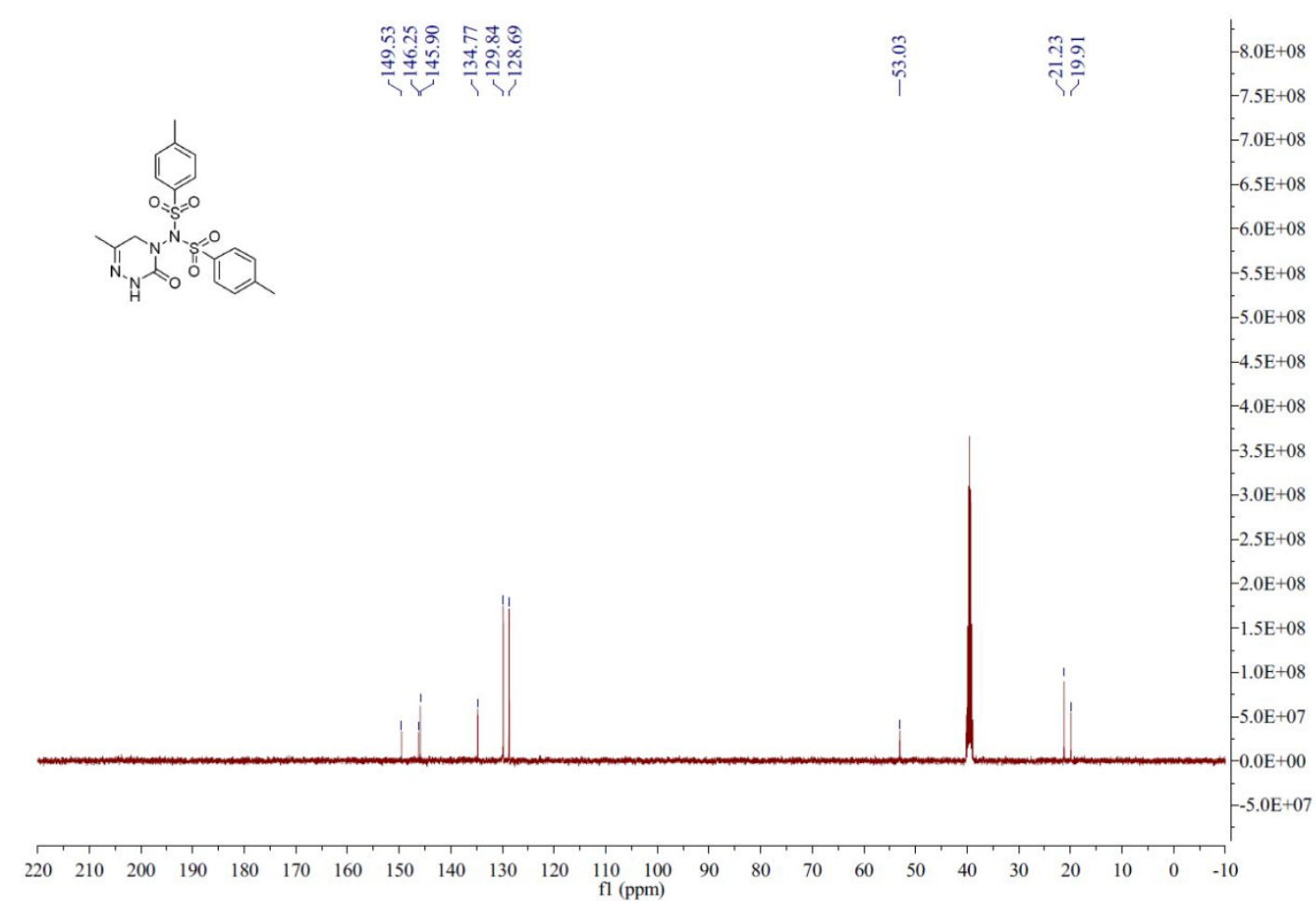

S55 
II-7 ${ }^{1} \mathrm{H}$ NMR (300 MHz, DMSO- $\left.d_{6}\right)$

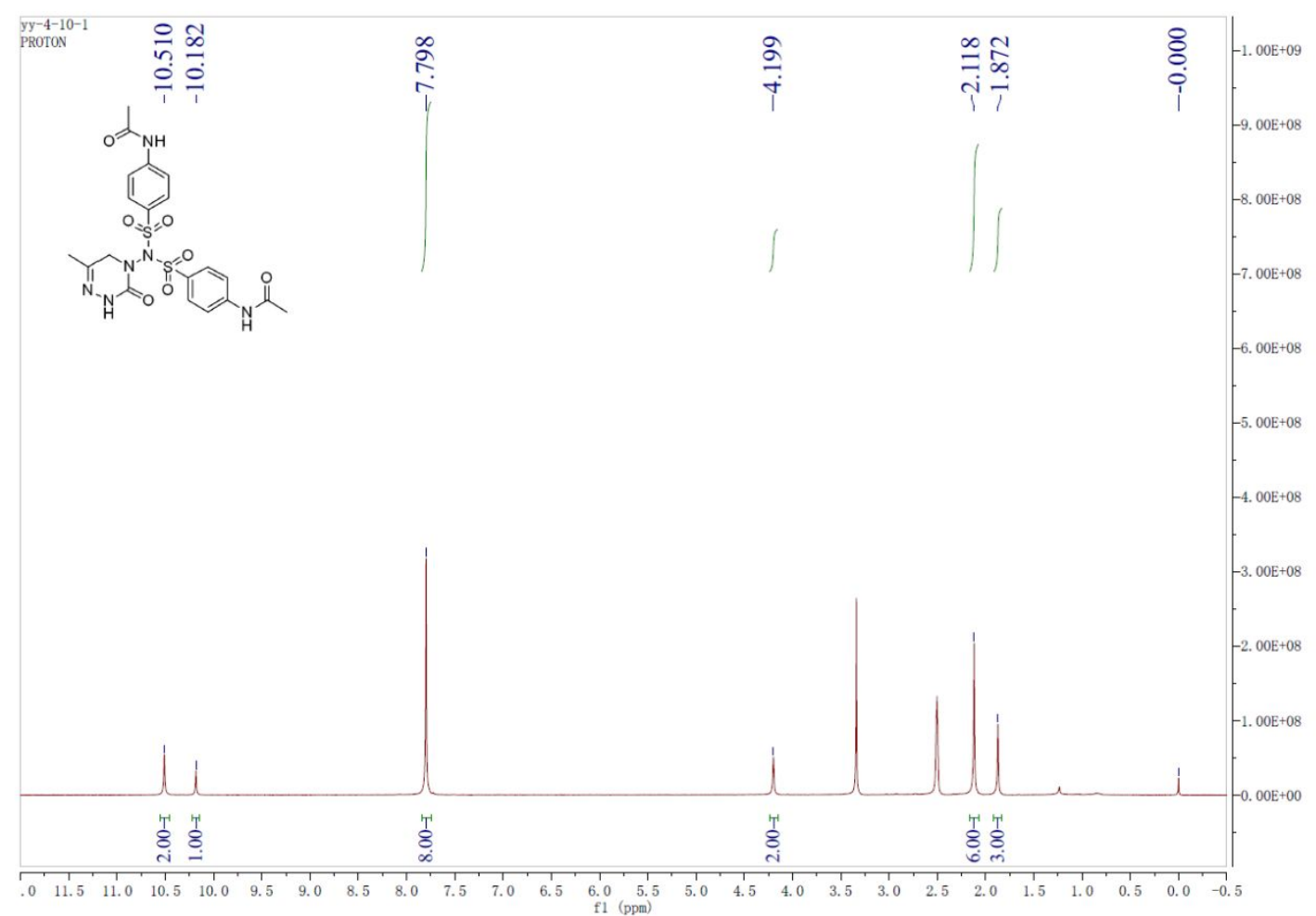

II-7 ${ }^{13} \mathrm{C}$ NMR (100 MHz, DMSO- $\left.d_{6}\right)$

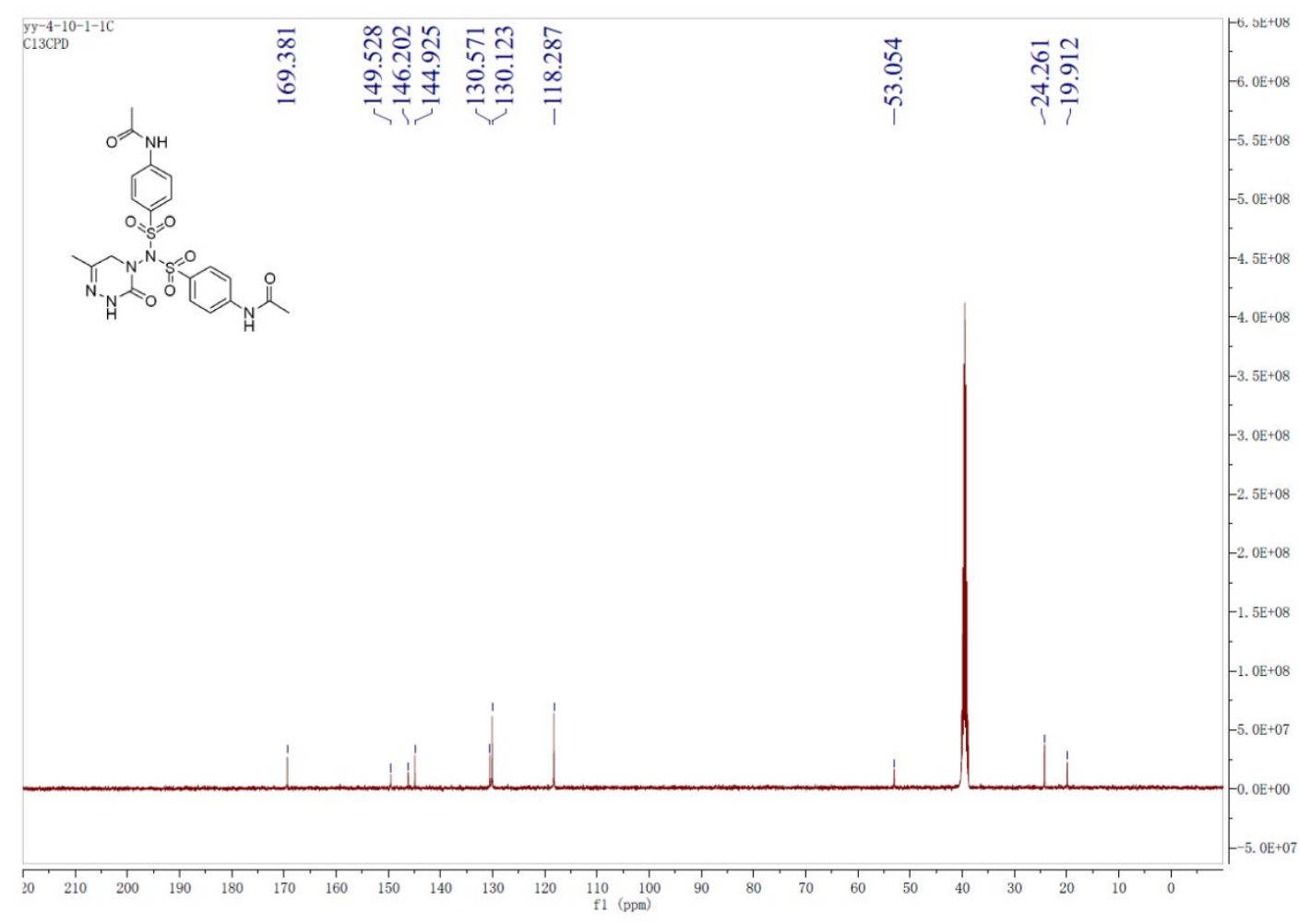


II-8 ${ }^{1} \mathrm{H}$ NMR (300 MHz, DMSO- $\left.d_{6}\right)$

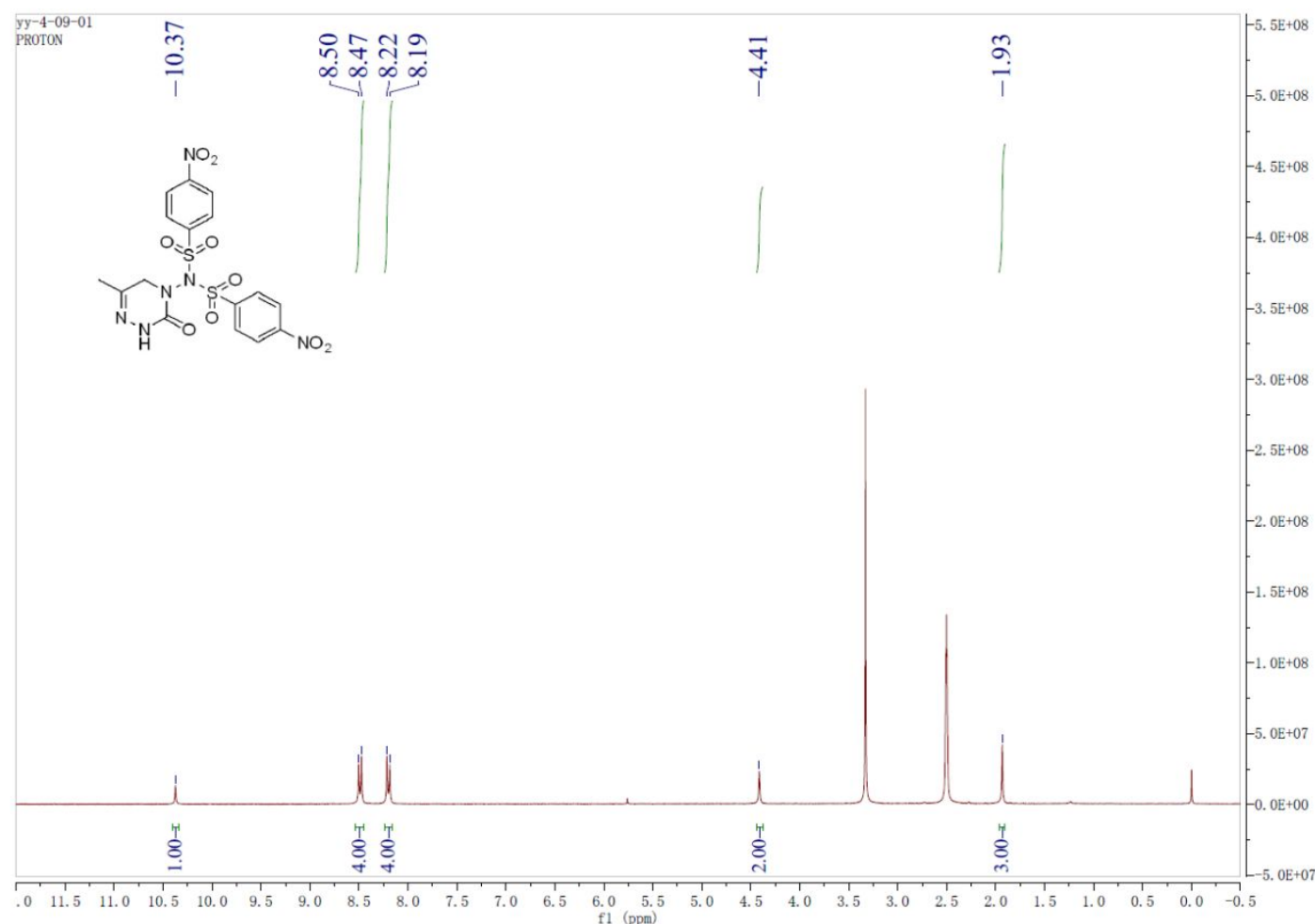

II-8 ${ }^{13} \mathrm{C}$ NMR (100 MHz, DMSO- $\left.d_{6}\right)$

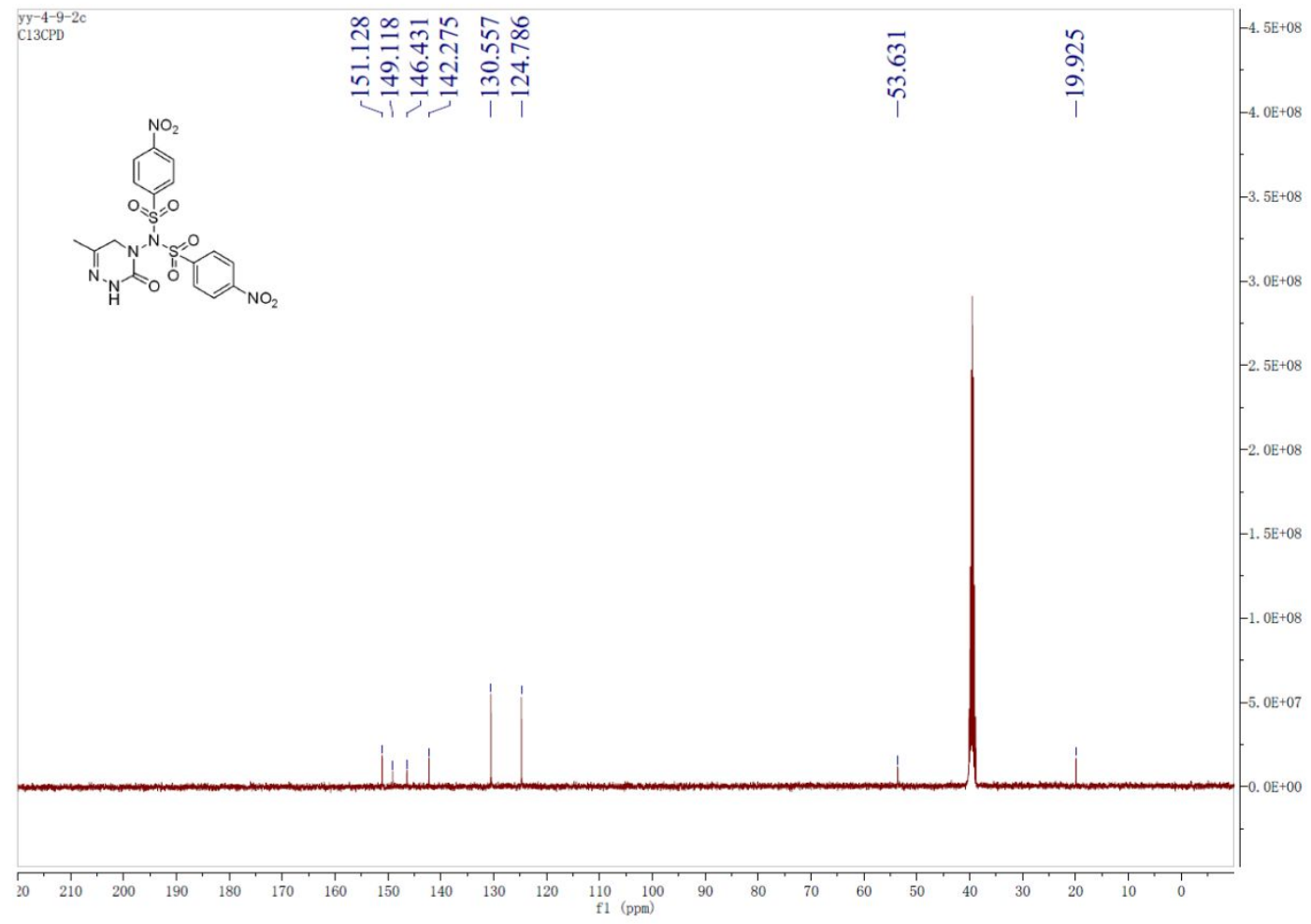




\section{Biological Assay}

All bioassays were performed on representative test organisms reared in the laboratory. The bioassay was repeated in triplicate at $25 \pm 1{ }^{\circ} \mathrm{C}$. Assessments were made on a dead/alive basis, and mortality rates were corrected using Abbott's formula. Evaluations were based on a percentage scale of $0-100$, where 0 equals no activity and 100 equals total kill. For comparative purpose, pymetrozine was tested under the same conditions.

Foliar Contact Activity against Aphis craccivora. The insecticidal activities of the title compounds and the pymetrozine were tested against A. craccivora by foliar application. About 60 aphids were transferred to the shoot with 3-5 fresh leaves of horsebean. The shoot with aphids was cut and dipped into the test solution for $2 \mathrm{~s}$, after removing extra solutions on the leaf; the aphids were raised in the shoot at $25 \pm 1{ }^{\circ} \mathrm{C}$ and $85 \%$ relative humidity for $96 \mathrm{~h}$. Each experiment for one compound was triplicated. The revised death rate was calculated by Abbott's formula.

Larvicidal Activities against Mosquito (Culex pipiens pallens). 10 fourth-instar mosquito larvae were put into the $10 \mathrm{~mL}$ of the test solution. Percentage mortalities were evaluated 8 days after treatment. Evaluations were based on a percentage scale of $0-100$, where 0 equals no activity and 100 equals total kill. Each treatment was performed three times. Error of the experiments was about 5\%.

Stomach Toxicity against Helicoverpa armigera, Ostrinia nubilalis, and Mythimna separata.

The stomach toxicities of the title compounds against Helicoverpa armigera, Ostrinia nubilalis, and Mythimna separata were tested according to the leaf-dip method using the reported procedure. Leaf disks (about $5 \mathrm{~cm}$ ) were cut from fresh corn leaves and 
then were dipped into the test solution for 3-5 s. After air drying, the treated leaf disks were placed individually into a glass-surface vessel $(7 \mathrm{~cm})$. Each dried treated leaf disk was infested with 10 third-instar Mythimna separata, or Helicoverpa armigera, or Pyrausta nubilalis. Percentage mortalities were evaluated 4 days after treatment. Leaves treated with acetone were provided as controls. Each treatment was performed three times. 
The proposed mechanism

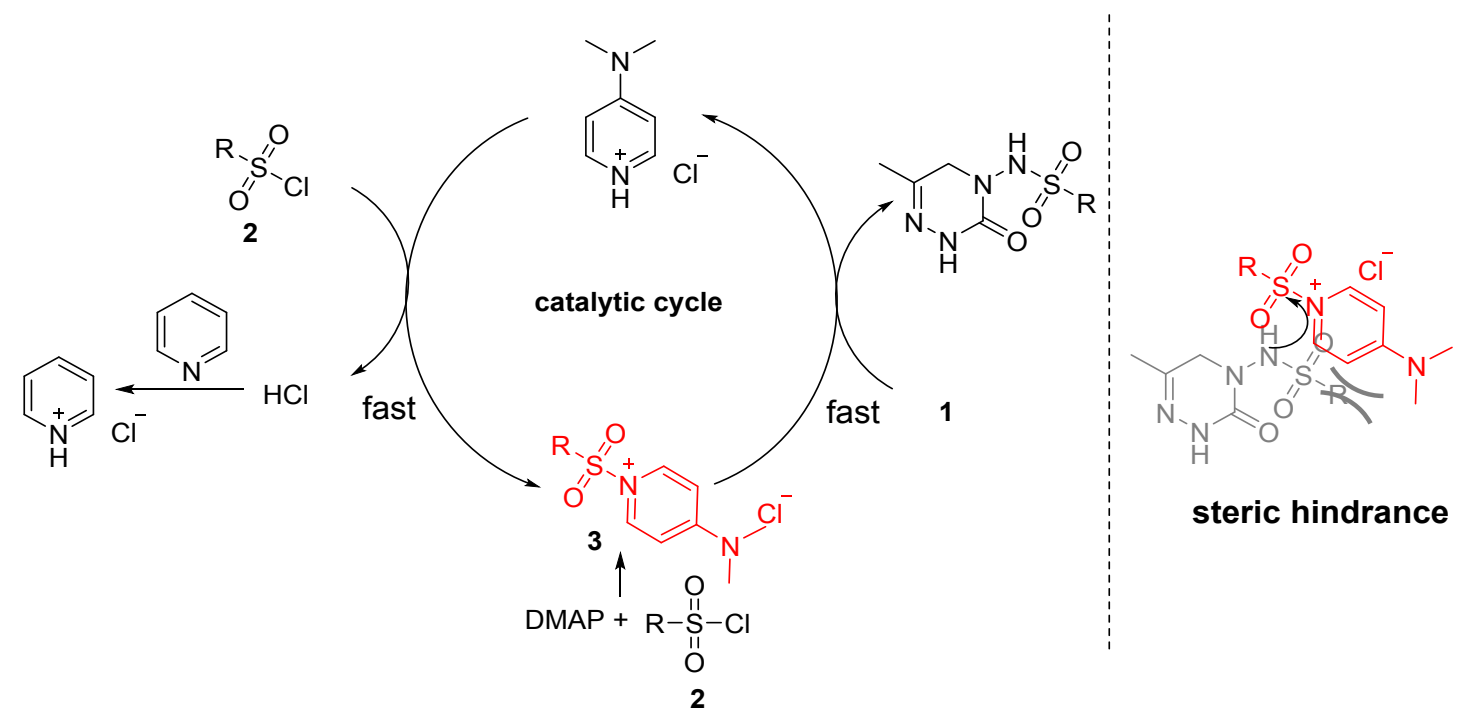

First, sulfonyl chloride 2 reacted with DMAP to form $\mathbf{3}$, and second, the nucleophilic substrate $\mathbf{1}$ attacks 3 to release the product and generates DMAP $\cdot \mathrm{HCl}$ (which is a very fast process). With the help of pyridine, DMAP $\cdot \mathrm{HCl}$ reacts with sulfonyl chloride to regenerate $\mathbf{3}$ (fast reaction) and complete the catalytic cycle. The steric hindrance prevents 3 from further reacting with monosulfonylated products to form bissulfonylated by-products. 